\title{
High Performance Microwave Integrated Filters for 5G Applications
}

by

Jiacheng Zhang

A thesis submitted to the Faculty of Graduate and Postdoctoral Affairs in partial fulfillment of the requirements for the degree of

Master of Applied Science

in

Electrical and Computer Engineering

Carleton University

Ottawa, Ontario

(C) 2019

Jiacheng Zhang 


\begin{abstract}
A $71-76 \mathrm{GHz}$ millimeter-wave filter is designed and implemented in a multilayer LTCC module to help attenuate the side spur signal level. The filter is aimed to be embedded in a novel E-band architecture designed at Huawei for millimeter-wave signal communication application. The goal of the filter is to help reduce the third harmonic and fourth harmonic leakage of the LO signal from tripler into the RF channel.
\end{abstract}

Through measurement, for $-15 \mathrm{dBm}$ received signal power, the $3^{\text {rd }}$ and $4^{\text {th }}$ harmonic leakage levels sof the LO signal is around $-25 \mathrm{dBc}$, and both of their frequency range are very close to the transmission band. The designed bandpass filter attenuates the $3^{\text {rd }}$ and $4^{\text {th }}$ harmonic signal power down to $-50 \mathrm{dBc}$ with a low filter order and small physical size.

Unfortunately, access to the fabricated filter was not possible due to existing political tension between the US government and Huawei. The fabricated filter was not shipped to Ottawa, and as a result, it was not measured.

A second bandpass filter operating at $10 \mathrm{GHz}$ is fabricated using Rogers 4360 substrate for measurement and validation. The measurement results show some deviations with simulation results. The center frequency shifts from $10 \mathrm{GHz}$ to $11.25 \mathrm{GHz}$. Through analysis, the milling depth of the fabrication process affects the measurement result. In our test, a $0.243 \mathrm{~mm}$ milling depth causes the filter center frequency shift to $11.25 \mathrm{GHz}$. 


\section{Acknowledgments}

First, I'd like to thank professor Rony Amaya and professor Jim Wight. As my supervisor and co-supervisor, they make a great contribution to my graduate study. I still remember when I took professor Jim Wight's PLL class, and I was fascinated by the way he teaches. So, at that time, I decided to become a research-based student. I must thank professor Amaya for providing me the funding and the chance to work in Huawei. As a Chinese citizen, I am so happy and very proud to work in this world-famous Chinese company.

I must thank Morris Repeta and Wenyao Zhai. Morris, as my manager in Huawei, assisted me in solving many problems from the start of my internship to the end. Wenyao, as my colleague and a former student of Carleton University, gives me a lot of guidance on filter design.

I want to thank Kerwin Johnson for reviewing my paper and giving me a lot of guidance. Besides that, I admire his attitude towards life very much. I am really impressed that he has never used a mobile phone in his life. It is not easy to live a quiet and undisturbed life today, but he did it.

There are also many other people I must give my thanks to. I must thank all the millimeterwave team member for their kindness help. This made me understand the importance of the team. Trying to ask for help is sometimes the right choice.

最后我要感谢我的父母, 感谢你们对我的教育, 定不负厚望。

iii 


\section{Table of Contents}

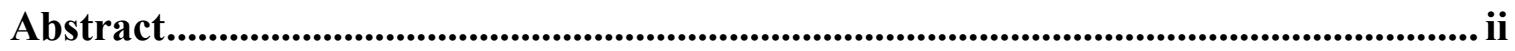

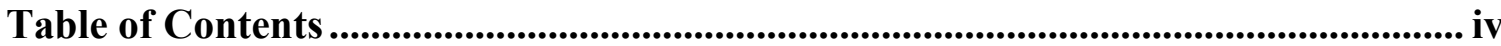

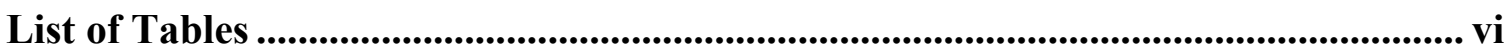

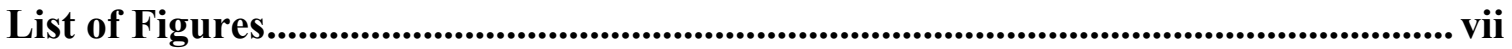

List of Abbreviations and Symbols ......................................................................................... xi

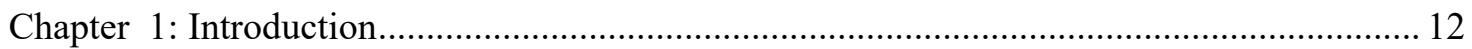

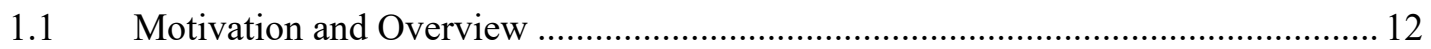

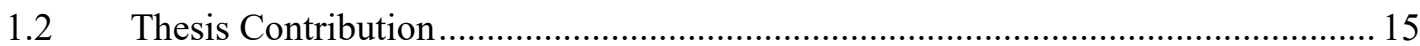

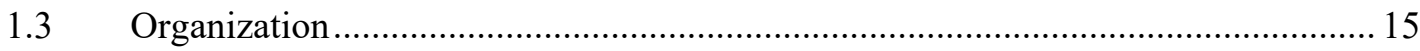

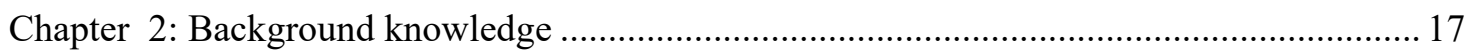

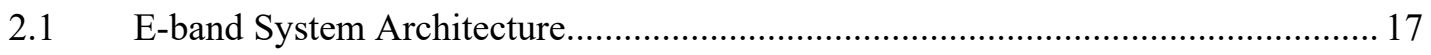

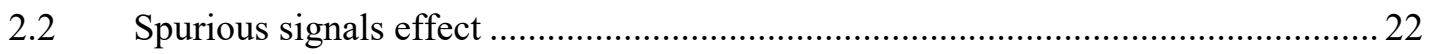

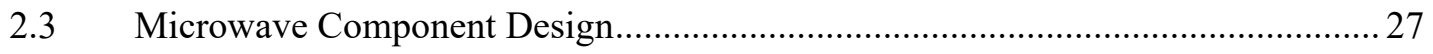

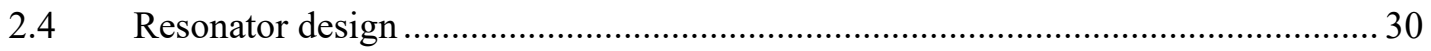

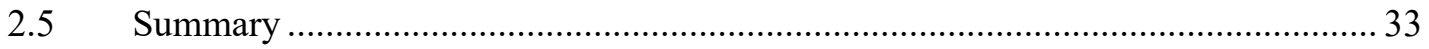

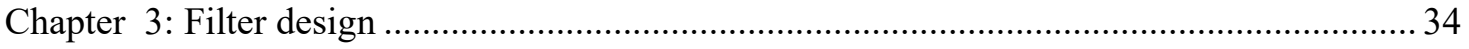

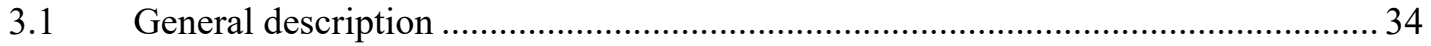

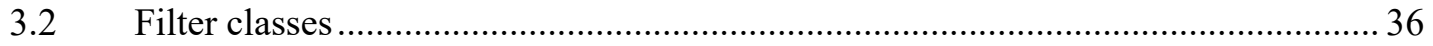

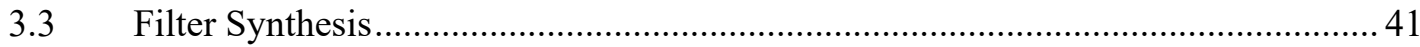

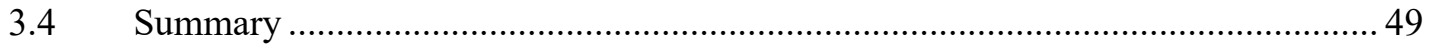

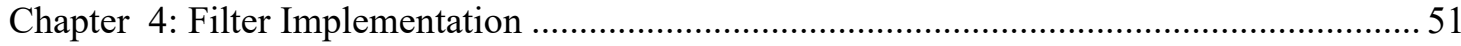

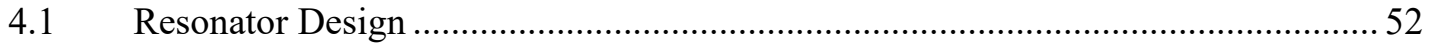

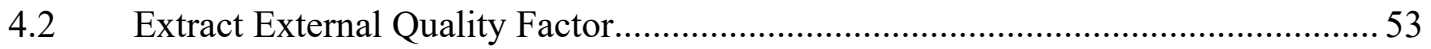




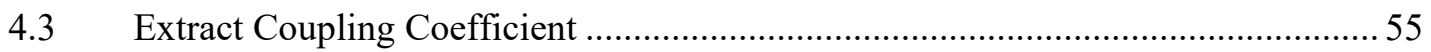

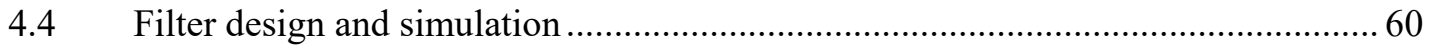

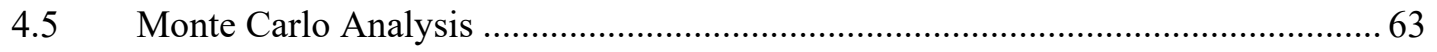

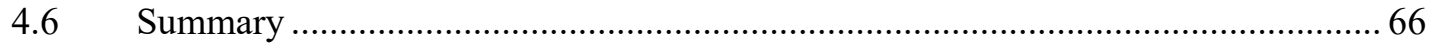

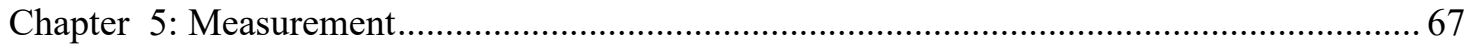

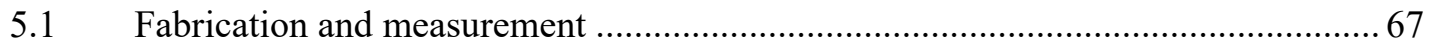

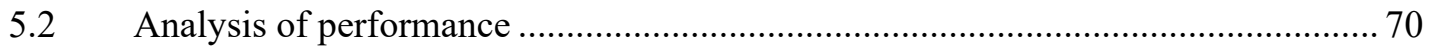

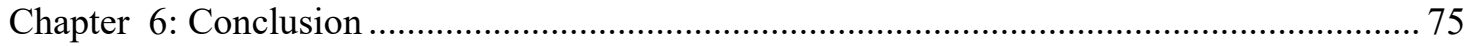

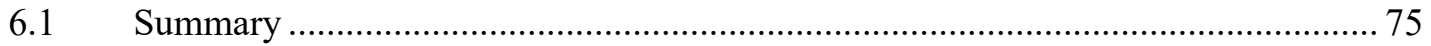

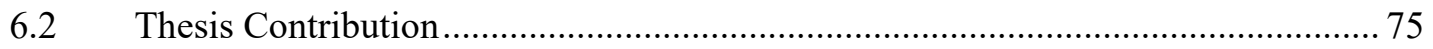

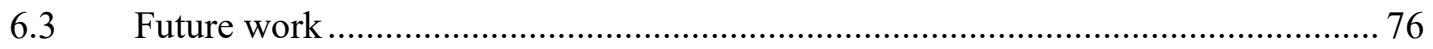

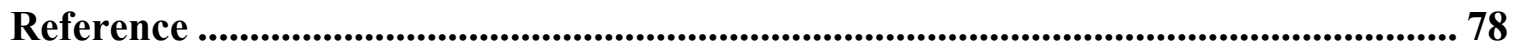




\section{List of Tables}

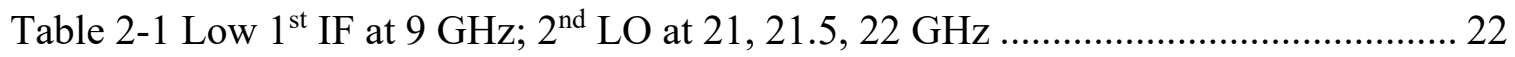

Table 2-2 Midband $1^{\text {st }} \mathrm{IF}$ at $11 \mathrm{GHz} ; 2^{\text {nd }} \mathrm{LO}$ at $20.33,20.83,21.33 \mathrm{GHz} \ldots \ldots \ldots \ldots \ldots \ldots . . . . . . . .23$

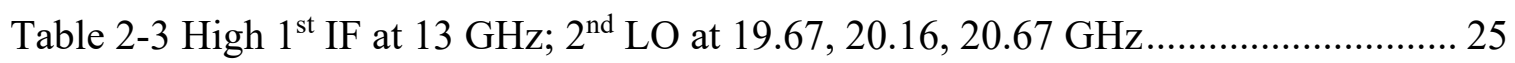

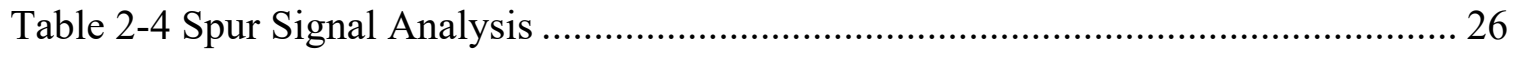

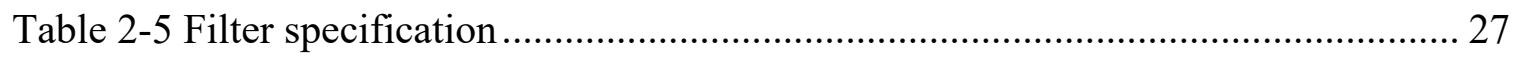

Table 3-1 Element Values for Butterworth Lowpass Prototype Filters [4] .................... 42

Table 3-2 Element Values for Chebyshev Lowpass Prototype Filters [4]..................... 43

Table 3-3 Element values for Four-Pole Prototype $(\boldsymbol{L R}=-\mathbf{2 0 d B})$............................. 44

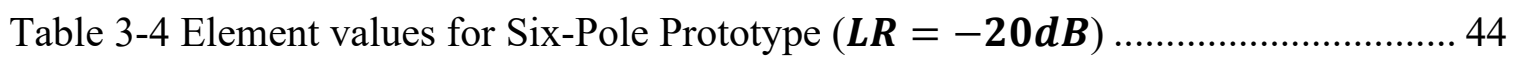

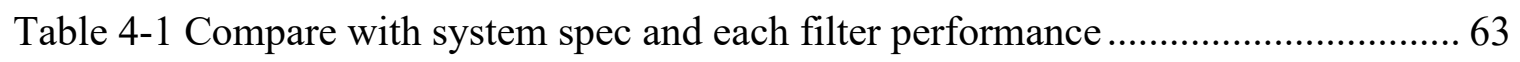

Table 5-1 Simulation Versus Measurement............................................................ 70 


\section{List of Figures}

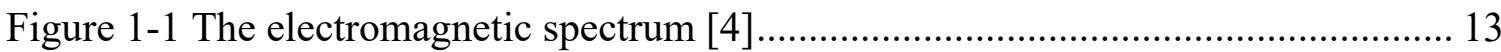

Figure 1-2 Rain attenuation in $\mathrm{dB} / \mathrm{km}$ across frequency at various rainfall rates[5] ....... 14

Figure 1-3 atmospheric absorptions across mm-wave frequencies in $\mathrm{dB} / \mathrm{km}[6] \ldots \ldots \ldots \ldots . . .14$

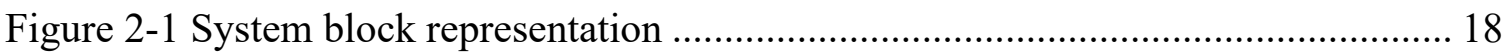

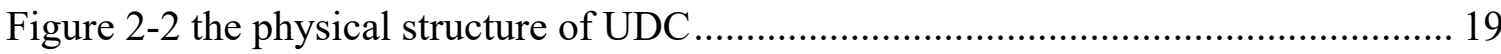

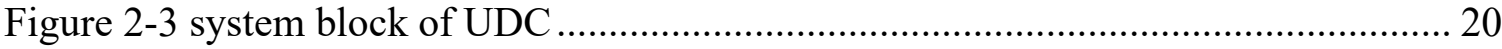

Figure 2-4 the physical structure of Active Antenna ...................................................... 21

Figure 2-5 System block of active antenna...................................................................... 21

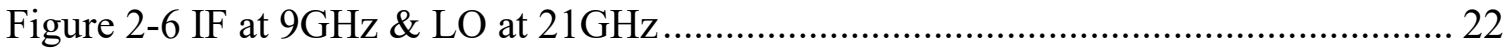

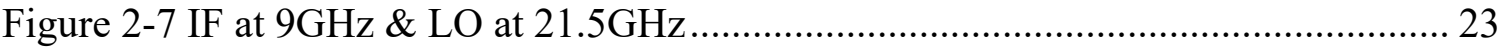

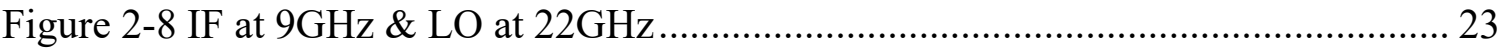

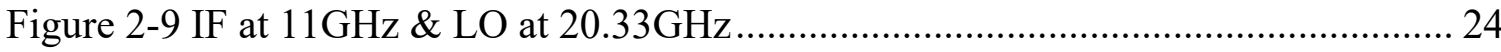

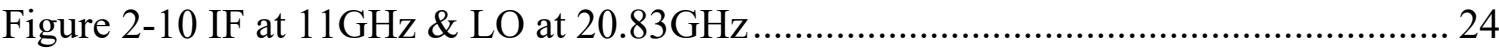

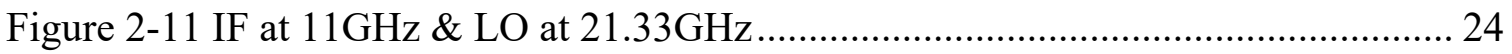

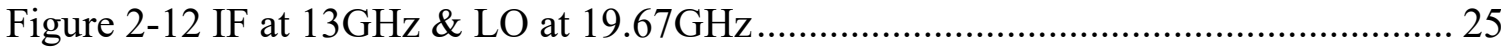

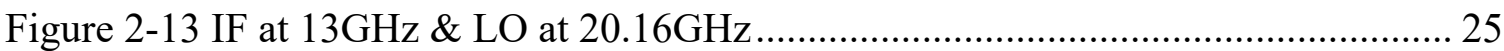

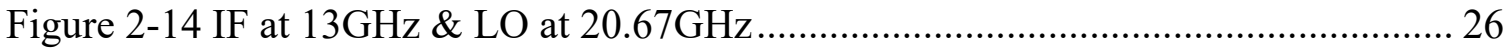

Figure 2-15 Lumped element Transmission Line model................................................ 28

Figure 2-16 geometry of a microstrip line (b) sketch of the field lines ............................ 28

Figure 2-17 (a) geometry of stripline (b) sketch of the field lines.................................... 29

Figure 2-18 Lumped Series Resonator Model ........................................................... 31

Figure 2-19 Lumped Parallel Resonator Model................................................................ 32 vii 
Figure 2-20 Transmission Line Terminated at an open-end ........................................ 32

Figure 3-1 Butterworth lowpass prototype frequency response …............................ 37

Figure 3-2 Attenuation versus normalized frequency for different order Butterworth

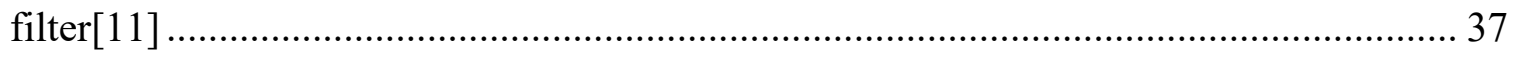

Figure 3-3 Chebyshev lowpass prototype frequency response .................................. 38

Figure 3-4 Attenuation versus normalized frequency for Chebyshev filter[11] ............. 39

Figure 3-5 Elliptic lowpass prototype frequency response .................................... 40

Figure 3-6 Compare with Chebyshev and the filter with a signal pair of attenuation pole at

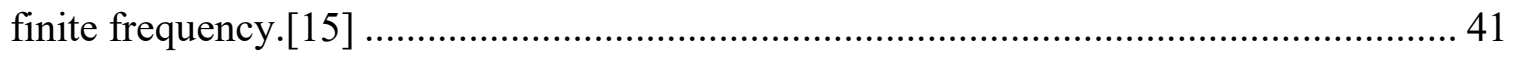

Figure 3-7 Lowpass prototype for Butterworth and Chebyshev filter .......................... 42

Figure 3-8 Lowpass prototype for quasi-elliptic filter[13] ................................... 43

Figure 3-9 Lowpass prototype to bandpass transformation ..................................... 46

Figure 3-10 Equivalent circuits of n-coupled resonators circuit................................ 47

Figure 3-11 General Coupling structure for a bandpass filter with a pair of transmission

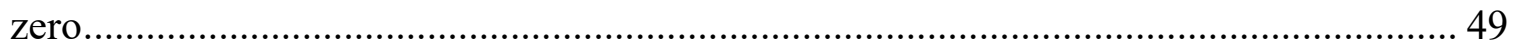

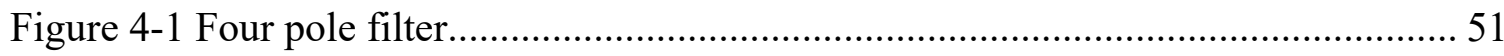

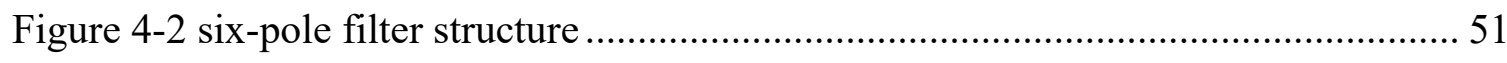

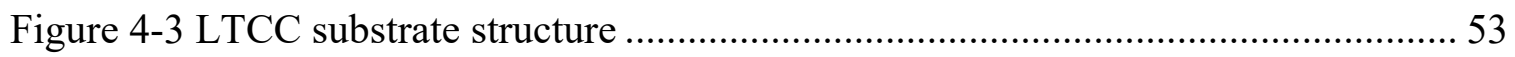

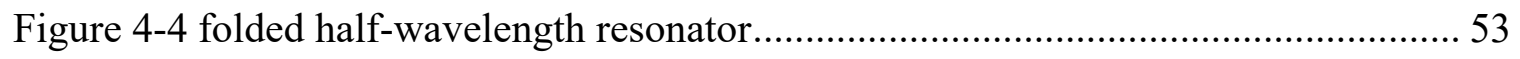

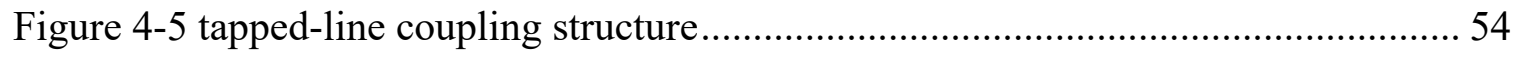

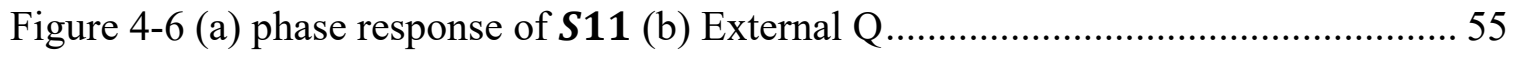

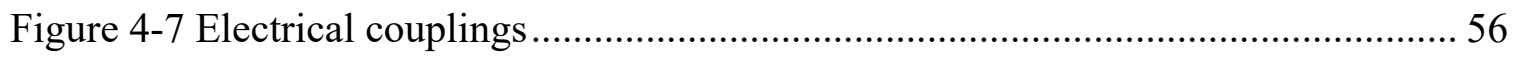

Figure 4-8 (a) frequency response; (b) Coupling Coefficient...................................... 57 viii 
Figure 4-9 Magnetic Coupling..... 58

Figure 4-10 (a) Frequency Response of Magnetic Coupling ; (b) Coupling Coefficient

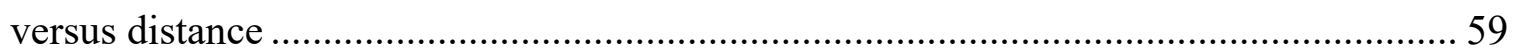

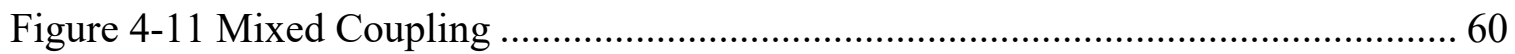

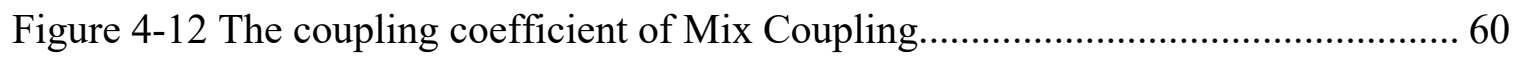

Figure 4-13 Four pole filter............................................................................... 61

Figure 4-14 Frequency response of Four Pole Filter ............................................. 61

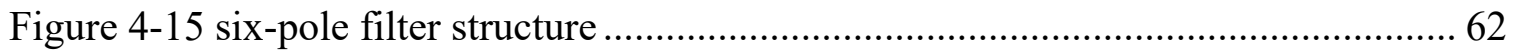

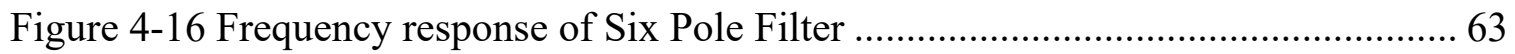

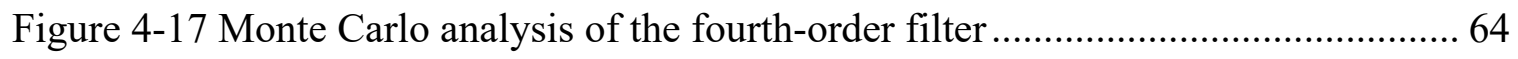

Figure 4-18 100 trials of a fourth-order filter Monte Carlo analysis .............................. 65

Figure 4-19 Monte Carlo Analysis for the sixth order filter ..................................... 65

Figure 4-20 100 trials of sixth-order filter Monte Carlo analysis ................................. 66

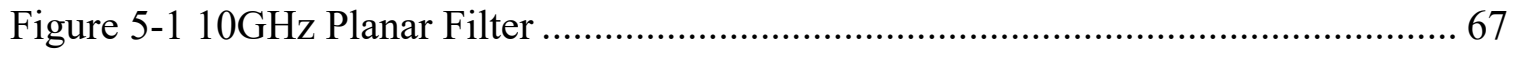

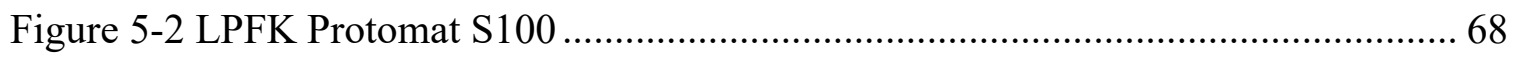

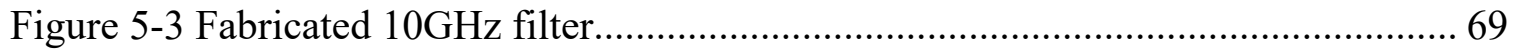

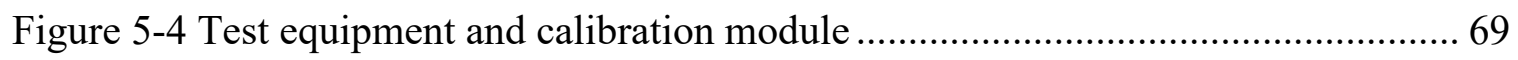

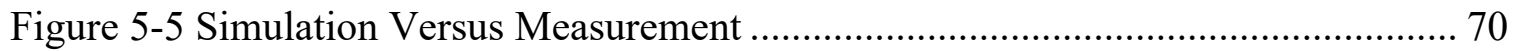

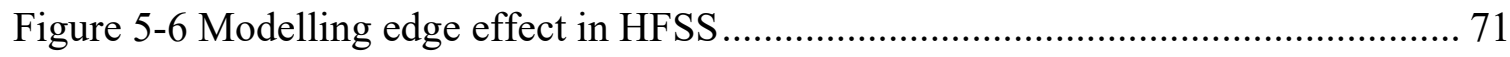

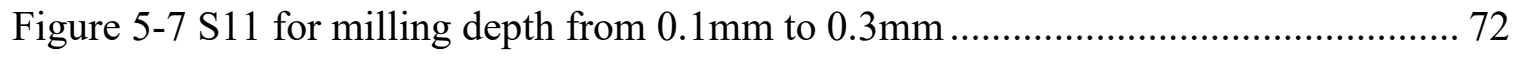

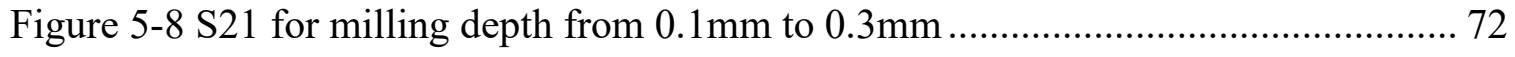

Figure 5-9 Measuring the milling depth of the fabricated filter ................................ 73

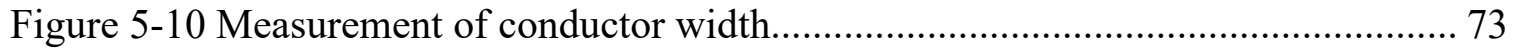
ix 
Figure 5-11 0.243mm milling depth simulation result compare with the measurement result

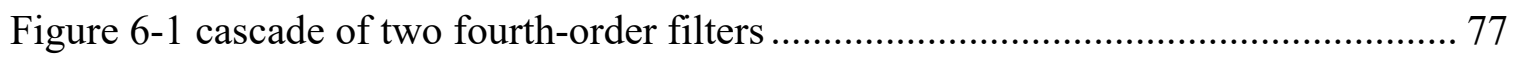

Figure 6-2 Frequency response of the filter with two pair of transmission zero ............. 77 


\section{List of Abbreviations and Symbols}

$5 \mathrm{G}$

AA

ADS

$\mathrm{F}_{\mathrm{n}}$

FBW

$g_{n}$

$\mathrm{k}_{\mathrm{E}}$

$\mathrm{k}_{\mathrm{M}}$

$\mathrm{L}_{\mathrm{A}}$

$\mathrm{L}_{\mathrm{R}}$

LNA

LTCC

PA

Q

RF

SIW

UDC

$\mathrm{Z}_{\text {in }}$

$\mathrm{Z}_{\mathrm{o}}$

$\omega_{0}$

$\Omega_{\mathrm{a}}$
The fifth generation of wireless communication

Active Antenna

Advanced Design Software

Characteristic Function of filter prototype

Fractional bandwidth

Reactive component of a lowpass prototype

Electrical Coupling Coefficient

Magnetic Coupling Coefficient

Insertion Loss of two-port filter

Return Loss of two-port filter

Low Noise Amplifier

Low-Temperature Co-fired Ceramic

Power Amplifier

Quality Factor of Resonator

Radio Frequency

Substrate Integrated Waveguide

Up and Down Converter

Input impedance

Characteristic Impedance

Center frequency of Resonator

Transmission zero location 


\section{Chapter 1: Introduction}

\subsection{Motivation and Overview}

The fifth-generation $(5 \mathrm{G})$ wireless standard has already become a reality. Based on recent VNI report and forecast, in just a decade, the amount of IP data handled by the wireless network will have increased by well over a factor of 100, from under 3 exabytes in 2010 to over 190 exabytes by 2018 , on pace to exceed 500 exabytes by 2020 [1]. In addition to the sheer volume of data, the number of devices and the data rates will continue to grow exponentially. The number of devices could reach the tens or even hundreds of billions by the time $5 \mathrm{G}$ comes to fruition, due to many new applications beyond personal communications[2]. Such a rapid increase in mobile data growth and the use of smartphones are creating unprecedented challenges for wireless service providers to overcome a global bandwidth shortage.

\subsubsection{Millimeter-Wave}

The global bandwidth shortage facing wireless carriers has motivated the exploration of the underutilized millimeter wave frequency spectrum[3] for future broadband cellular communication networks. Figure [1-1] shows the location of the RF and millimeter-wave frequency bands in the electromagnetic spectrum and their typical applications. The millimeter-wave frequency range is between $30 \mathrm{GHz}$ to $300 \mathrm{GHz}$, where the wavelength is from $1-10 \mathrm{~mm}$. 


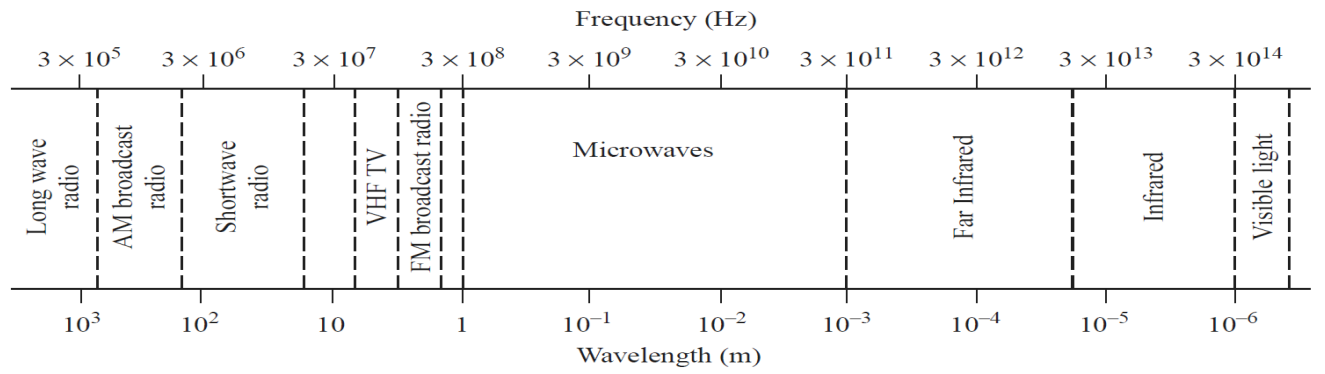

Typical Frequencies

AM broadcast band Short wave radio band FM broadeast band VHF TV (2-4) VHF TV (5-6) UHF TV $(7-13)$ UHF TV (14-83) US cellular telephone

European GSM cellular GPS

Microwave ovens US DBS

US ISM bands

US UWB radio

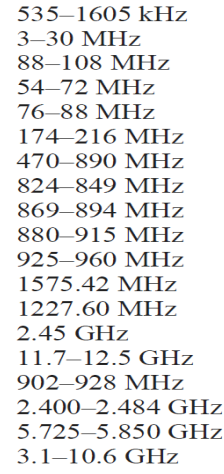

Approximate Band Designations

Medium frequency $\quad 300 \mathrm{kHz}-3 \mathrm{MHz}$ High frequency $(\mathrm{HF}) \quad 3 \mathrm{MHz}-30 \mathrm{MHz}$ Very high frequency (VHF) $30 \mathrm{MHz}-300 \mathrm{MHz}$ Ultra high frequency (UHF) $300 \mathrm{MHz}-3 \mathrm{GHz}$ L band

$\mathrm{S}$ band

$\mathrm{C}$ band

$\mathrm{X}$ band

X band

Ku band

$\mathrm{K}$ band

Ka band

$\mathrm{U}$ band

$\mathrm{V}$ band

E band

W ban

1-2 GHz

2- $4 \mathrm{GH} z$

4-8 GHz

8-12 GHz

$12-18 \mathrm{GHz}$

$18-26 \mathrm{GHz}$

$26-40 \mathrm{GHz}$

$26-40 \mathrm{GHz}$
$40-60 \mathrm{GHz}$

$50-75 \mathrm{GHz}$

60-90 GHz

$75-110 \mathrm{GHz}$

F band

Figure 1-1 The electromagnetic spectrum [4]

In the past, Millimeter-wave bands were not a good option for mobile communications because of its poor transmission qualities, including strong path loss, atmospheric and rain absorption, low diffraction around obstacles, and penetration through objects. Figure [1-2] and Figure [1-3] show the rain attenuation and atmospheric absorption characteristics of mm-wave propagation. 


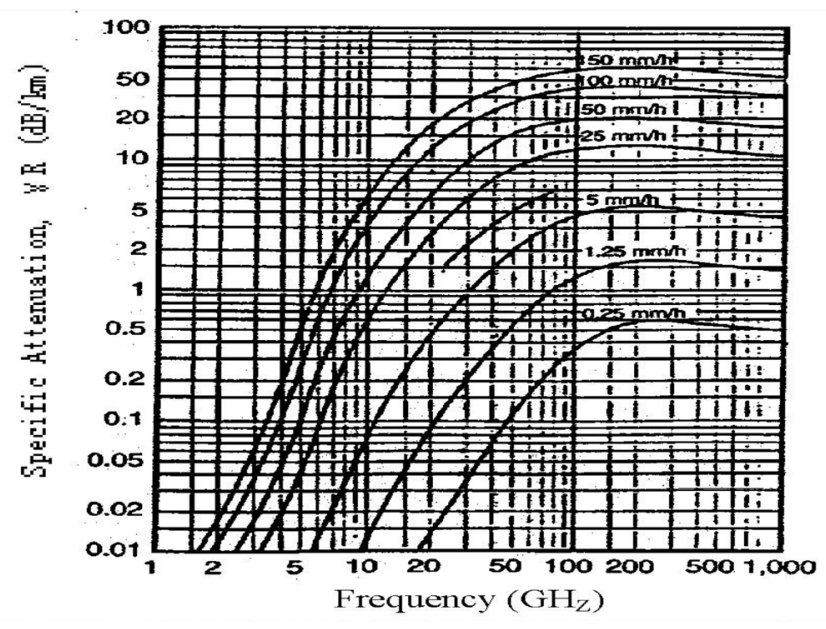

Figure 1-2 Rain attenuation in $\mathrm{dB} / \mathrm{km}$ across frequency at various rainfall rates[5]

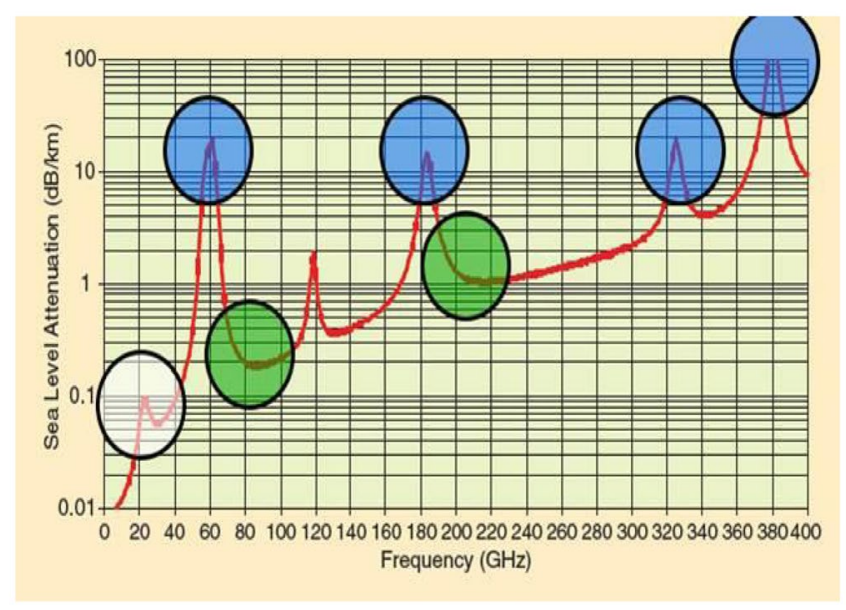

Figure 1-3 atmospheric absorptions across $\mathbf{m m}$-wave frequencies in $\mathbf{d B} / \mathbf{k m}[6]$

Due to the development and progress of the production technology level, and the birth of new wireless technologies, it is now possible to utilize millimeter-wave frequency band to transmit and receive signal since many disadvantages mentioned above now have been overcome. In [7], new transceiver designs at $60 \mathrm{GHz}$ are listed, which indicates that millimeter-wave signal transmission is feasible. 


\subsection{Thesis Contribution}

Communicating through millimeter-wave frequencies requires a new transceiver architecture, which includes all circuit components design such as LNA, mixers, filters, and PA. In this thesis, two different order bandpass filters operating at $71-76 \mathrm{GHz}$ are designed and implemented in an LTCC module, one is forth order, and one is sixth order. The size of the fourth-order filter is $0.6 \mathrm{~mm} \times 0.6 \mathrm{~mm}$, and the size of the six-order filter is $1.2 \mathrm{~mm} \times 0.6 \mathrm{~mm}$. Both filters provide more than $25 \mathrm{~dB}$ attenuation at 0.1 offset frequency normalized to the cutoff frequency at 1 . The in-band insertion loss is around $5 \mathrm{~dB}$, mainly due to the high conductor loss at $70 \mathrm{GHz}$.

However, the fabricated filters cannot be shipped back to Ottawa for measurement. As a substitution, another filter fabricated with Rogers $4360 \mathrm{G} 2$ materials operating at $10 \mathrm{GHz}$ using the same technology is fabricated and measured for design validation.

\subsection{Organization}

Chapter 2 presents all the necessary background knowledge. A novel transceiver architecture transmitting and receiving the EM signal through E-band frequency is first described on system level. The transceiver structure divides into two parts, the up and down converter (UDC) and the active antenna (AA). Detail structures of each part are described. The design equations for microstrip and stripline structure design are given for transmission line resonator design purpose. 
Chapter 3 covers the background of the bandpass filter design. Starting from a general transfer function description, through lowpass prototype synthesis, frequency transformation, and circuit network equalization, a bandpass filter structure can be constructed and implemented through coupling theory. Coupling theory is a very useful filter design method, especially for narrow bandwidth bandpass filter design. It converts all design parameters into two parameters: the external quality factor and coupling coefficients. These two parameters are directly related to the physical structure of the filter and can be simulated through an EM simulator.

Chapter 4 is the detailed procedure for filter design with the assistance of commercial EM simulator. The design includes how to extract the external quality factor by changing the tapped line position and how to extract the electric and magnetic coupling coefficient by changing the gap distance between two resonators. Full-EM simulation software is necessary for this parameter extraction.

In chapter 5, a planar filter operating at $10 \mathrm{GHz}$ is designed and fabricated for measurement and validation. Since the processing technology is relatively old, the accuracy of the produced filter cannot be guaranteed. There is a signification deviation between the test result and the simulation result. We analyzed the causes of these deviations and made a conclusion. 


\section{Chapter 2: Background knowledge}

In order to meet the $5 \mathrm{G}$ standard and the data transmitting rate requirement, millimeterwave communication is now a mainstream research direction. A new type of transceiver designed by Huawei Technology can transmit a signal at the E-band $(71-76 \mathrm{GHz})$ frequency and use array antenna technology to enhance signal strength and reduce signal interference.

\subsection{E-band System Architecture}

Figure [2-1] is an E-band transceiver board including an LTCC module containing E-band transceiver chips with a phased array antenna, an onboard discrete IF link for signal up and down-conversion and an FPGA for control. It is a superheterodyne transceiver structure which includes two stages of frequency translation. The first IF is centered at around $11 \mathrm{GHz}$. The baseband signal, connected to the board through SMA connector or fiber, after upconversion, filtering and gain control, will be transmitted to the E-band chips for a second upconversion to $70 \mathrm{GHz}$ for radiation. The FPGA controls all other chips on the board, including the phase shifters on the E-band chips for phased array beam steering and power gain control on the IF link. There are two onboard PLLs, one feeding a fixed LO signal to the IF link and one feeding a $20 \mathrm{GHz}$ tunable LO signal to the E-band chips. The E-band module is the most critical component of the entire RF system, and a more detailed introduction will be provided in the next section. 


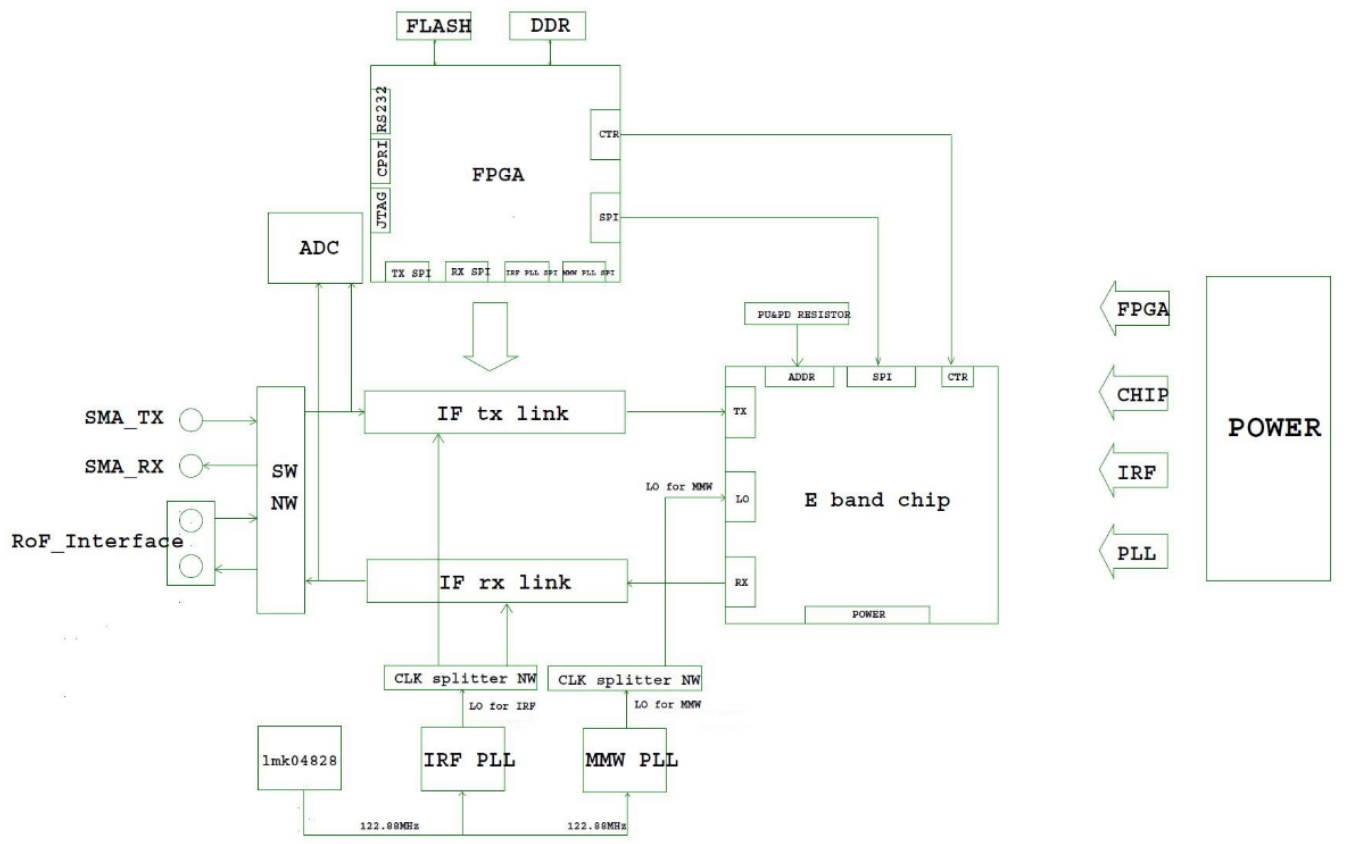

Figure 2-1 System block representation

\subsubsection{E-band module}

The E-band module is a nineteen-layer LTCC module with a 256-element phased array antenna and 32 channels of transmitting and receiving paths. Due to a multilayer approach, the LTCC module offers the possibility to integrate all the digital control, RF signal, and power lines into one module. The RF parts of this module contain two important components. One is the up and down converter chip (UDC) for the purpose of up and downconverting the signal between $70 \mathrm{GHz}$ and $11 \mathrm{GHz}$. Another one is the active antenna chip (AA) for the purpose of power control and phase adjustment of each path.

The physical structure of the UDC is shown in Figure [2-2]. Its system block representation is in Figure [2-3]. The incoming $20 \mathrm{GHz}$ Lo signal will be tripled and the $60 \mathrm{GHz}$ third 
harmonic is picked up for mixing purpose. The upconverting and mixing process is highly non-linear, and several unwanted harmonic signals might be generated. In the later section, some measurements will be presented, and there is a strong third and fourth harmonic of the LO signal leaking into the RF channel. The purpose of this thesis is to design a filter for suppressing those harmonic signals. The IF signal will be mixed with the third harmonic from the tripler and the outcome will be filtered and amplified, then sent to the antenna through a power divider for radiation.

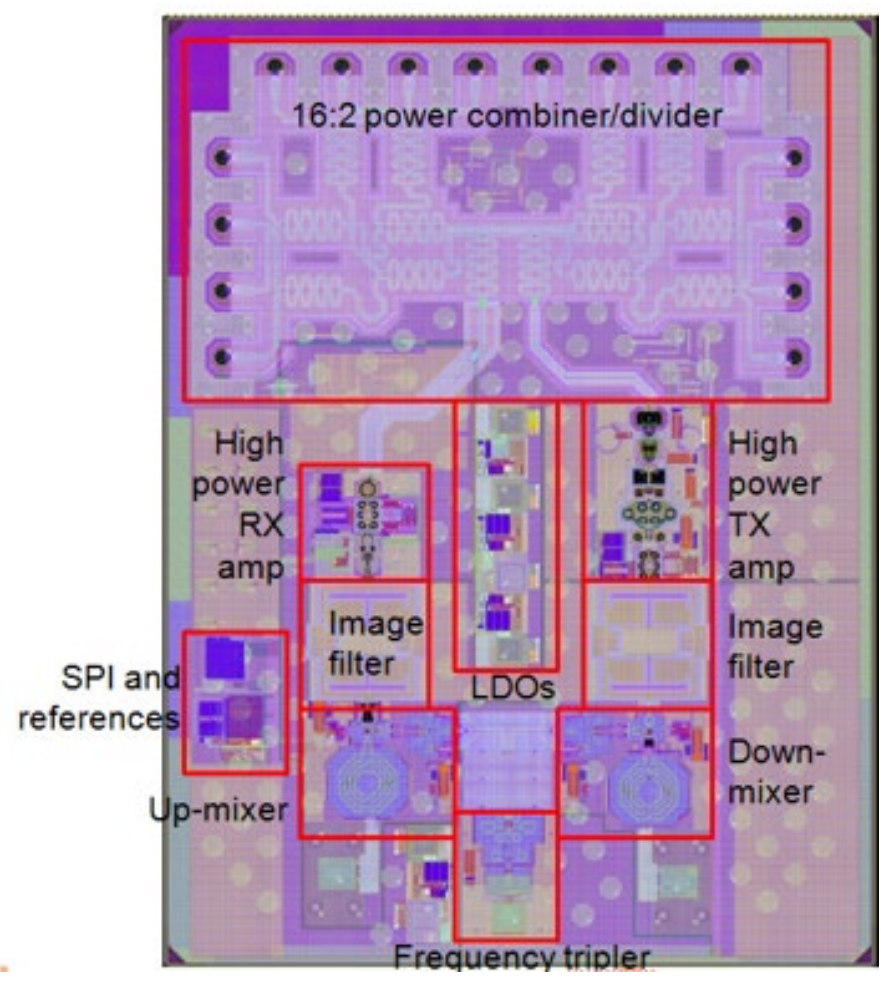

Figure 2-2 the physical structure of UDC 

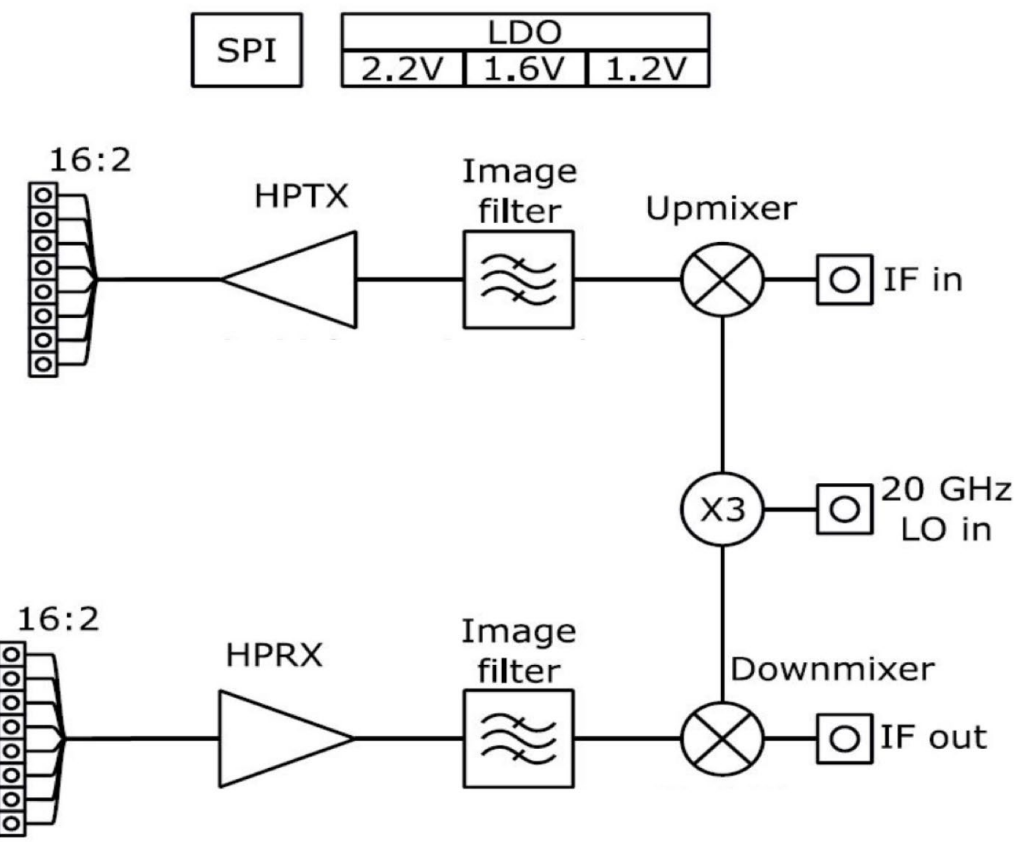

Figure 2-3 system block of UDC

Figure [2-4] is the physical structure of the active antenna part, and Figure [2-5] is its system block representation. It contains eight paths of transmitting channel and eight paths of receiving channel. Each channel has an amplifier and a phase shifter for power and phase correction. Each channel drives an 8-element subarray[8][9] of the phased array. The beam is steered by adjusting the relative phase of subarrays to create a gradient across the array. Control values for each beam are stored in a table and transmitted to the AA chips from the FPGA through a serial programming interface (SPI). 


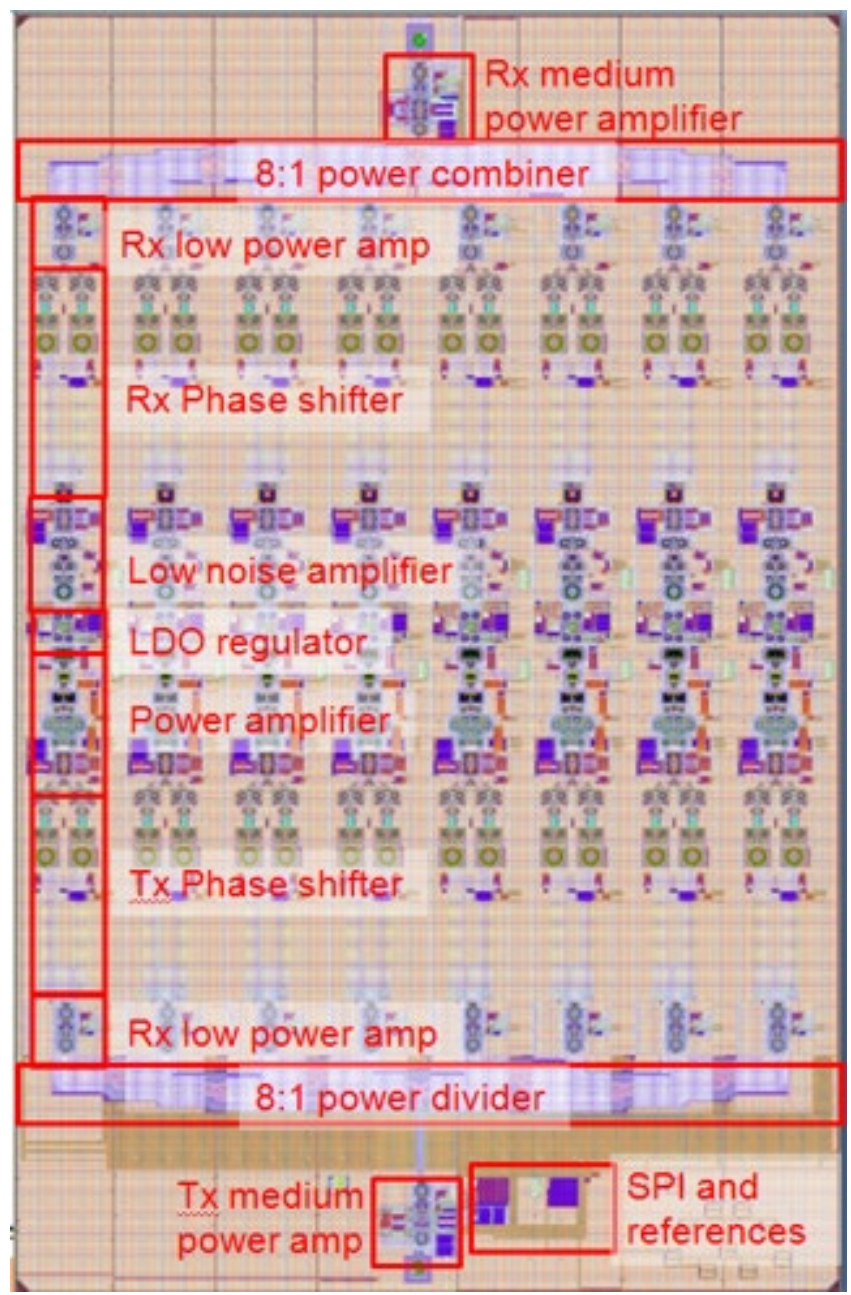

Figure 2-4 the physical structure of Active Antenna chip

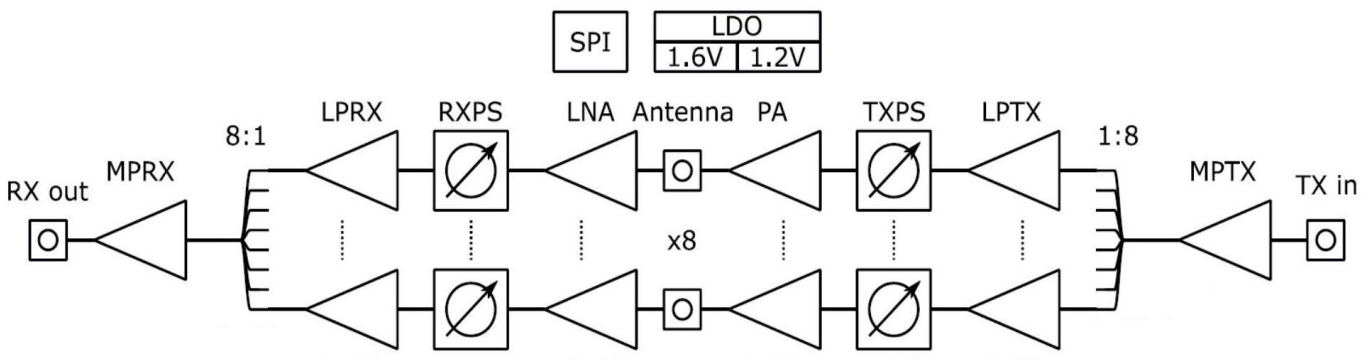

Figure 2-5 System block of active antenna chip 


\subsection{Spurious signals effect}

During the process of extracting the third harmonic of the LO signal, several unwanted spurs will also be generated, and some of them will leak into the RF path. In addition, the mixing process will also create some unwanted intermodulation signals between harmonics of IF and LO signal. It is necessary to determine the power levels of these unwanted spurious signals and keep them below a certain power level. A spur depression filter is required to do the job. In this architecture, three different IF frequencies were applied with various LO signals and measure the RF output. The transmitted IF power is $-15 \mathrm{dBm}$, and the received RF power is $-13 \mathrm{dBm}$. The spur signal power results are summarized in Table 2-1 to 2-3. Each Table has three Figures to illustrate how these spurs will change with different IF and LO frequency.

Table 2-1 Low $1^{\text {st }}$ IF at $9 \mathrm{GHz} ; 2^{\text {nd }} \mathrm{LO}$ at $21,21.5,22 \mathrm{GHz}$

\begin{tabular}{|c|c|c|c|c|c|c|c|c|c|}
\hline & $\mathrm{IF} / \mathrm{GHz}$ & $\mathrm{LO} / \mathrm{GHz}$ & $\mathrm{RF} / \mathrm{GHz}$ & $\begin{array}{c}4 \mathrm{LO}- \\
\mathrm{IF}\end{array}$ & $3 L O$ & $4 L O$ & $\begin{array}{c}2 \mathrm{LO}+ \\
2 \mathrm{IF}\end{array}$ & $\begin{array}{c}3 \mathrm{LO}+ \\
2 \mathrm{IF}\end{array}$ & $\begin{array}{c}5 \mathrm{LO}- \\
2 \mathrm{IF}\end{array}$ \\
\hline Freq & 9 & 21 & 72 & 75 & 63 & 84 & 60 & 81 & 87 \\
\hline Power $(\mathrm{dBc})$ & & & & -34 & -29 & -30 & null & -45 & null \\
\hline Freq & 9 & 21.5 & 73.5 & 77 & 64.5 & 86 & 61 & 82.5 & 89.5 \\
\hline Power $(\mathrm{dBc})$ & & & & -36 & -26.3 & null & null & -41 & null \\
\hline Freq & 9 & 22 & 75 & 79 & 66 & 88 & 62 & 84 & 92 \\
\hline Power $(\mathrm{dBc})$ & & & & -29 & -23 & null & -51.8 & -44 & null \\
\hline
\end{tabular}

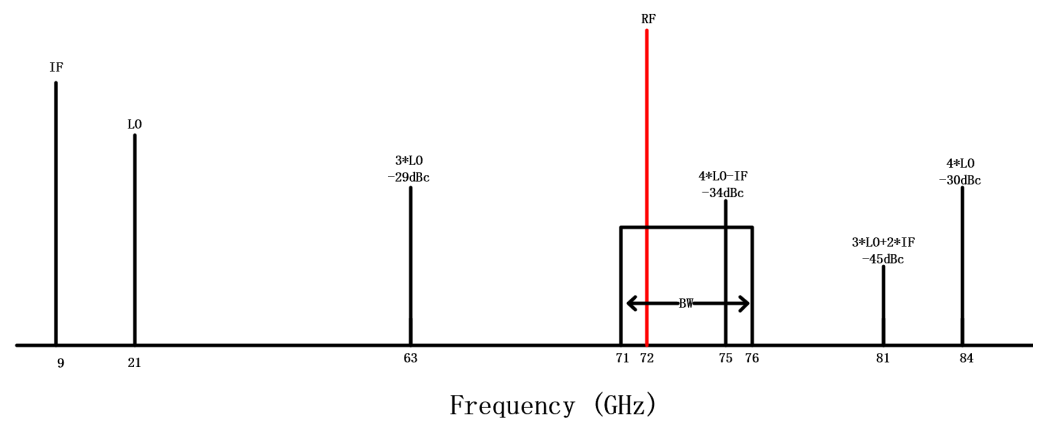

Figure 2-6 IF at $9 \mathrm{GHz} \& \mathrm{LO}$ at $21 \mathrm{GHz}$ 


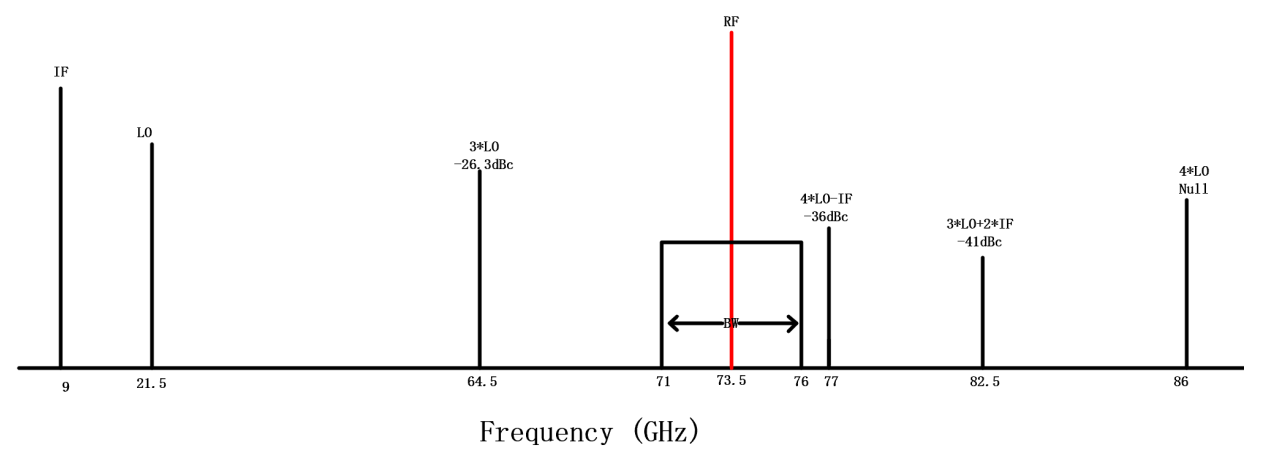

Figure 2-7 IF at 9GHz \& $\mathrm{LO}$ at $21.5 \mathrm{GHz}$

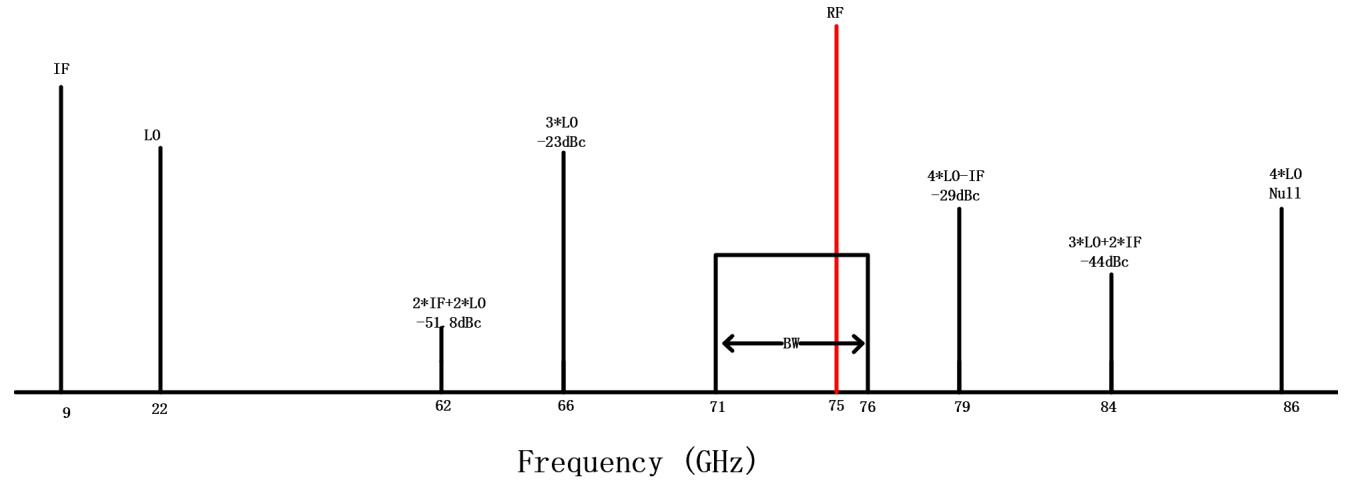

Figure 2-8 IF at $9 \mathrm{GHz} \& \mathrm{LO}$ at $22 \mathrm{GHz}$

Table 2-2 Midband $1^{\text {st }}$ IF at $11 \mathrm{GHz} ; 2^{\text {nd }} \mathrm{LO}$ at $20.33,20.83,21.33 \mathrm{GHz}$

\begin{tabular}{|l|c|c|c|c|c|c|c|c|c|}
\hline & $\mathrm{IF} / \mathrm{GHz}$ & $\mathrm{LO} / \mathrm{GHz}$ & $\mathrm{RF} / \mathrm{GHz}$ & $\begin{array}{c}4 \mathrm{LO}- \\
\mathrm{IF}\end{array}$ & $3 L O$ & $4 L O$ & $\begin{array}{c}2 \mathrm{LO}+ \\
2 \mathrm{~F}\end{array}$ & $\begin{array}{c}3 \mathrm{~L} 0^{+} \\
2 \mathrm{IF}\end{array}$ & $\begin{array}{c}5 \mathrm{LO}- \\
2 \mathrm{IF}\end{array}$ \\
\hline Freq & 11 & 20.33 & 71.99 & 70.32 & 60.99 & 81.32 & 62.66 & 82.99 & 79.65 \\
\hline Power (dBc) & & & & -39 & -36 & -27 & -41 & -46 & -50 \\
\hline Freq & 11 & 20.83 & 73.49 & 72.32 & 62.49 & 83.32 & 63.66 & 84.49 & 82.15 \\
\hline Power (dBc) & & & & -35 & -26 & -31 & nu11 & -45 & nu11 \\
\hline Freq & 11 & 21.33 & 74.99 & 74.32 & 63.99 & 85.32 & 64.66 & 85.99 & 84.65 \\
\hline Power (dBc) & & & & -40 & -28 & -32 & -41 & -46 & nul1 \\
\hline
\end{tabular}




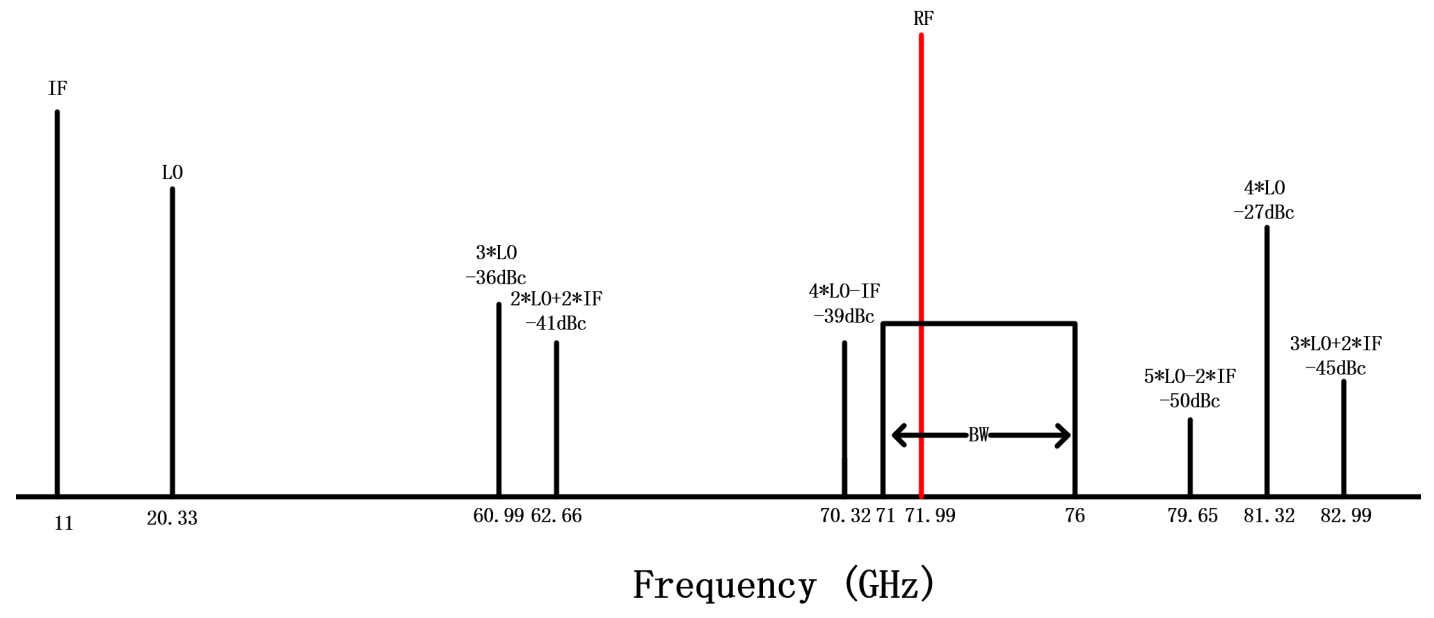

Figure 2-9 IF at $11 \mathrm{GHz} \& \mathrm{LO}$ at $20.33 \mathrm{GHz}$

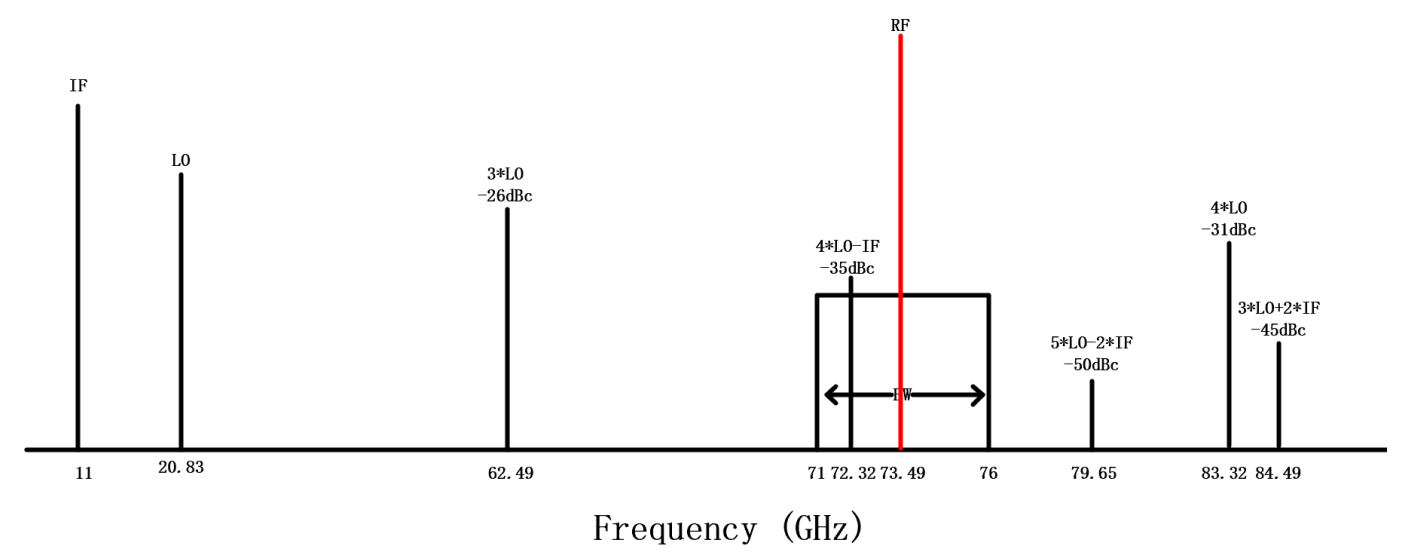

Figure 2-10 IF at $11 \mathrm{GHz} \& \mathrm{LO}$ at $20.83 \mathrm{GHz}$

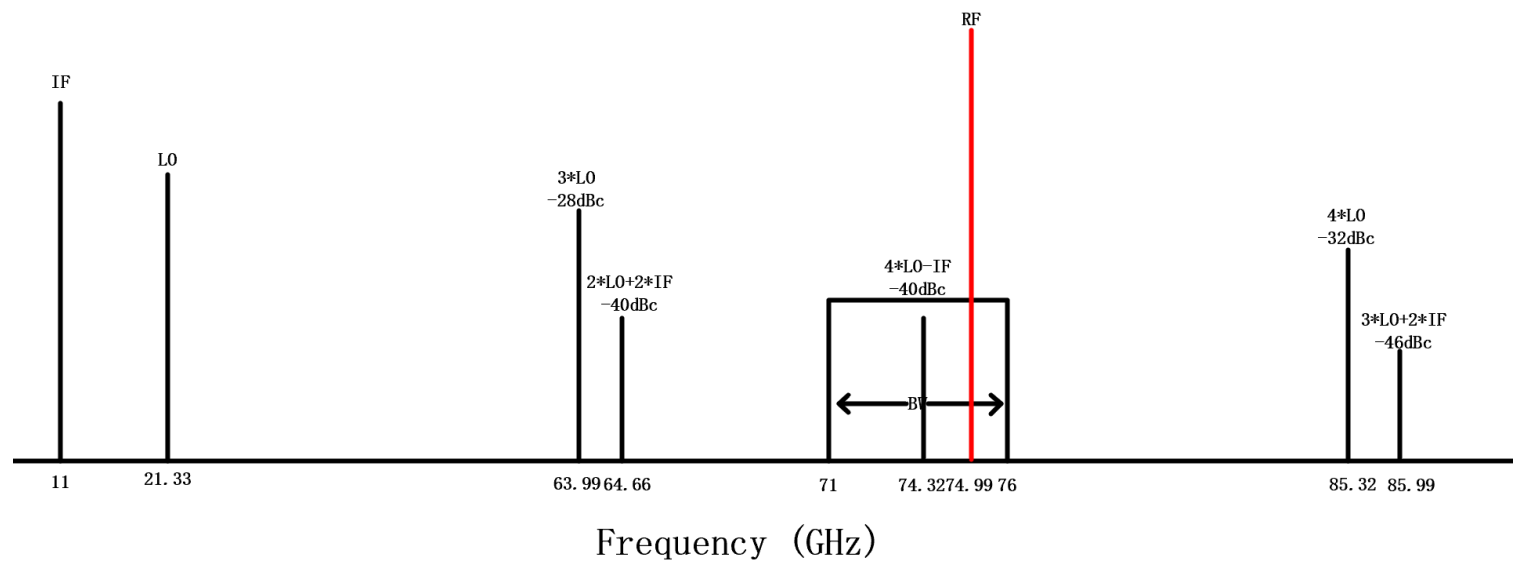

Figure 2-11 IF at $11 \mathrm{GHz} \& \mathrm{LO}$ at $21.33 \mathrm{GHz}$ 
Table 2-3 High $1^{\text {st }}$ IF at $13 \mathrm{GHz} ; 2^{\text {nd }} \mathrm{LO}$ at $19.67,20.16,20.67 \mathrm{GHz}$

\begin{tabular}{|c|c|c|c|c|c|c|c|c|c|}
\hline & $\mathrm{IF} / \mathrm{GHz}$ & $\mathrm{LO} / \mathrm{GHz}$ & $\mathrm{RF} / \mathrm{GHz}$ & $\begin{array}{c}4 \mathrm{~L}^{-} \\
\mathrm{IF}\end{array}$ & $3 L O$ & $4 L O$ & $\begin{array}{c}2 \mathrm{LO}+ \\
2 \mathrm{IF}\end{array}$ & $\begin{array}{c}3 \mathrm{LO}+ \\
2 \mathrm{IF}\end{array}$ & $\begin{array}{c}5 \mathrm{~L} 0^{-} \\
2 \mathrm{IF}\end{array}$ \\
\hline Freq & 13 & 19.67 & 72.01 & 65.68 & 59.01 & 78.68 & 65.34 & 85.01 & 72.35 \\
\hline Power $(\mathrm{dBc})$ & & & & -44 & nu11 & -29 & -44 & -50 & -52 \\
\hline Freq & 13 & 20.16 & 73.48 & 67.64 & 60.48 & 80.64 & 66.32 & 86.48 & 74.8 \\
\hline Power $(\mathrm{dBc})$ & & & & -45 & nu11 & -27 & -43 & nul1 & -52 \\
\hline Freq & 13 & 20.67 & 75.01 & 69.68 & 62.01 & 82.68 & 67.34 & 88.01 & 77.35 \\
\hline Power $(\mathrm{dBc})$ & & & & -36 & -32 & -31 & -45 & nul1 & -53 \\
\hline
\end{tabular}

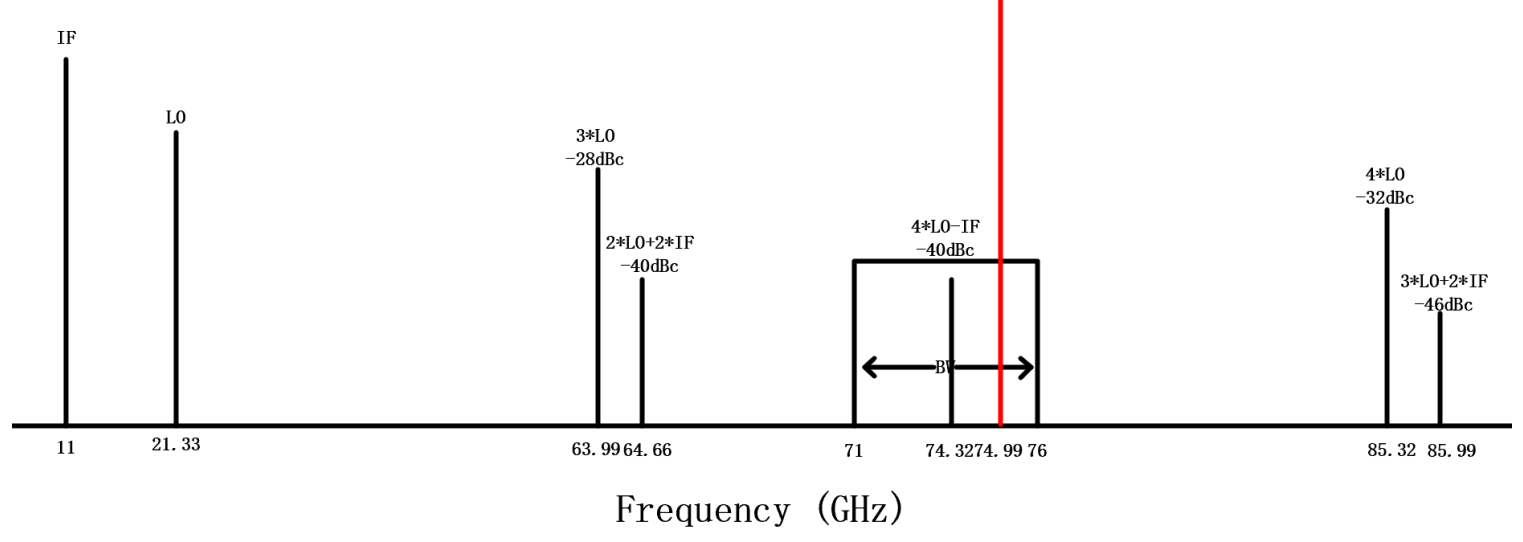

Figure 2-12 IF at $13 \mathrm{GHz} \& \mathrm{LO}$ at $19.67 \mathrm{GHz}$

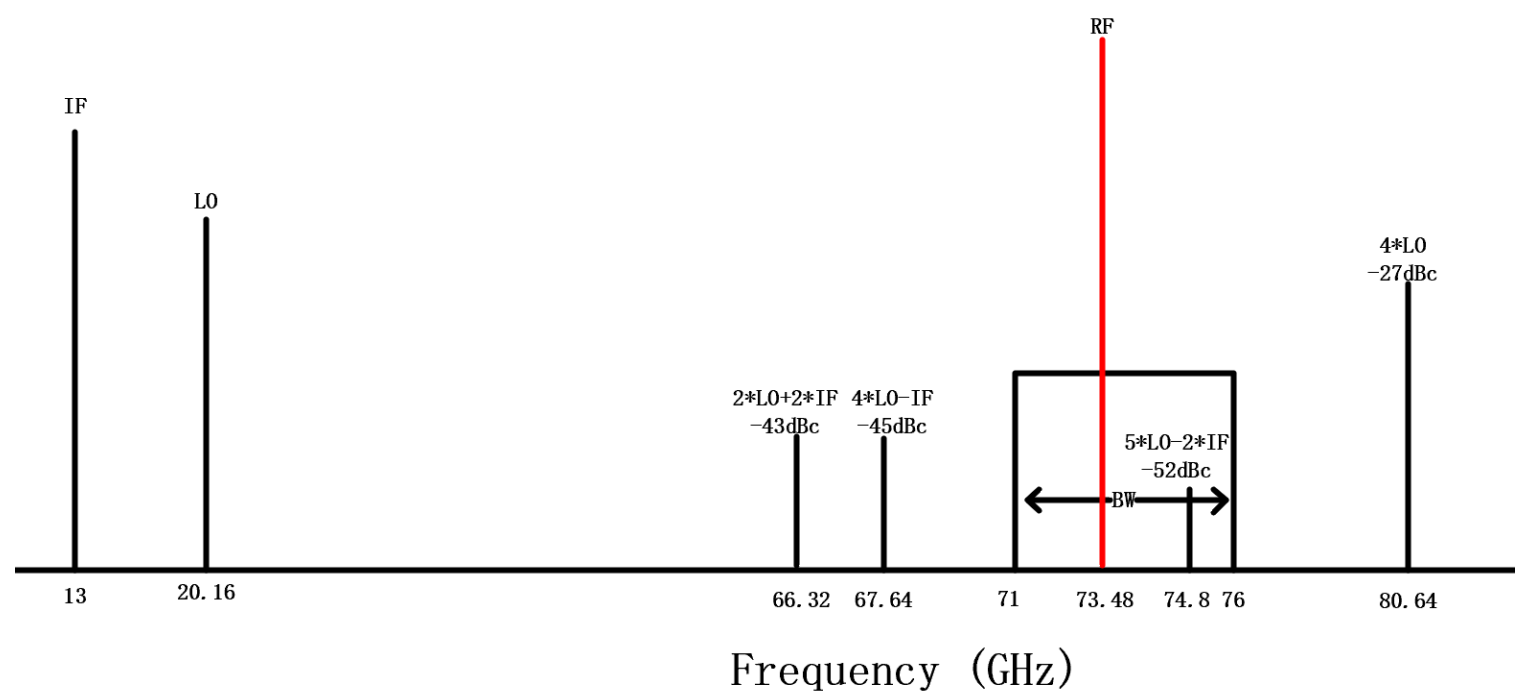

Figure 2-13 IF at $13 \mathrm{GHz} \& \mathrm{LO}$ at $20.16 \mathrm{GHz}$ 


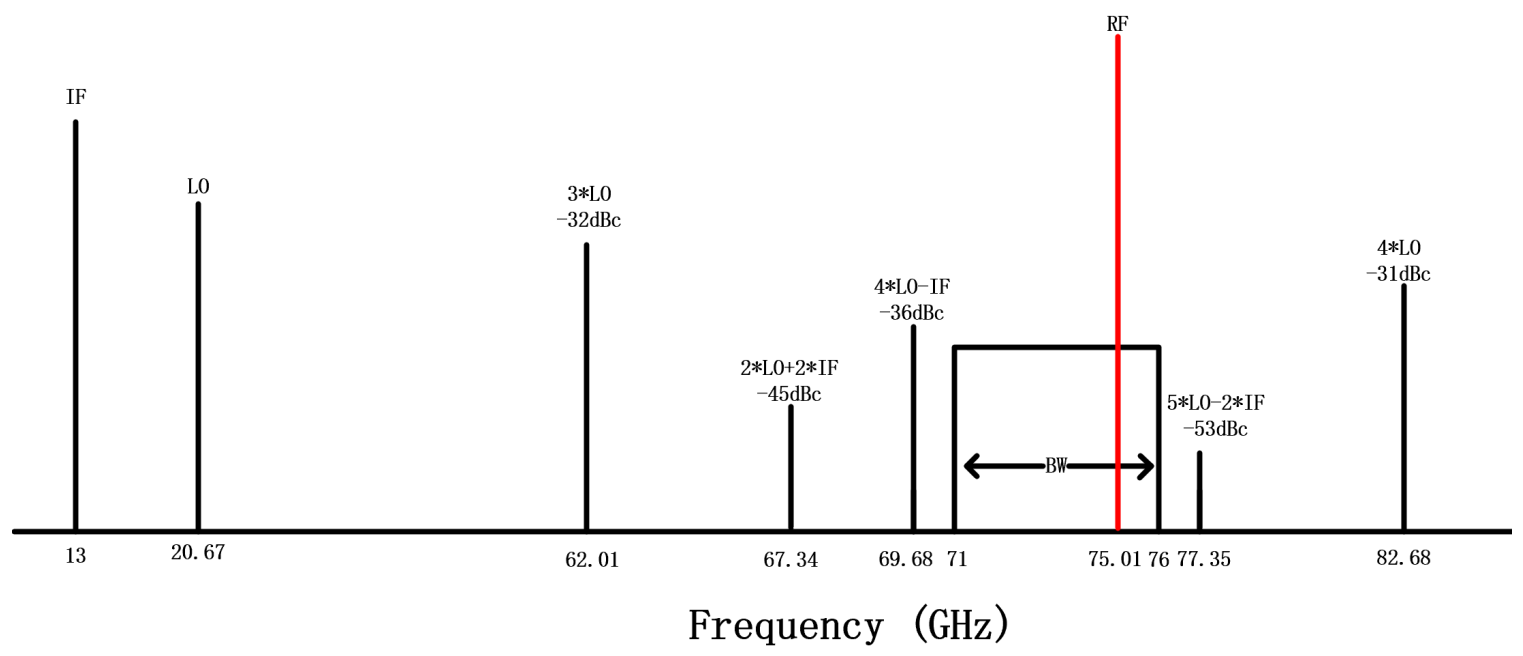

Figure 2-14 IF at $13 \mathrm{GHz} \& \mathrm{LO}$ at $20.67 \mathrm{GHz}$

Based on the measurement result, the summarization of all spur signal behavior is shown in Table [2-4] below:

\begin{tabular}{|c|c|c|c|}
\hline & Frequency Range $(\mathrm{GHz})$ & Max Power Level & Can be eliminated \\
\hline $4 * \mathrm{LO}-\mathrm{IF}$ & $65.68-79$ & $-34 \mathrm{dBc}$ & No \\
\hline $3 * \mathrm{LO}$ & $59.01-66$ & $-23 \mathrm{dBc}$ & Yes \\
\hline $4 * \mathrm{LO}$ & $78.68-88$ & $-27 \mathrm{dBc}$ & Yes \\
\hline $2 * \mathrm{LO}+2 * \mathrm{IF}$ & $60-67.34$ & $-41 \mathrm{dBc}$ & Yes \\
\hline $3 * \mathrm{LO}+2 * \mathrm{IF}$ & $81-88$ & $-41 \mathrm{dBc}$ & Yes \\
\hline $5 * \mathrm{LO}-2 * \mathrm{IF}$ & $72.35-92$ & $-50 \mathrm{dBc}$ & No \\
\hline
\end{tabular}

Table 2-4 Spur Signal Analysis

In conclusion, the $4 * \mathrm{LO}-\mathrm{IF}$ and the $5 * \mathrm{LO}-2 * \mathrm{IF}$ spur cannot be eliminated since they are often in the transmission bandwidth. The $2 * \mathrm{LO}+2 * \mathrm{IF}$ and the $3 * \mathrm{LO}+2 * \mathrm{IF}$ spurs can be easily eliminated since their power level is low, and they are far out of the band. The $3 * \mathrm{LO}$ and $4 * \mathrm{LO}$ have a very high-power level, and they are close to the bandwidth. Therefore, a sharp selectivity filter is required to attenuate the $3 *$ Lo and $4 *$ Lo harmonic. 26 
Based on these measurement results, we want to attenuate the spur signal power level down to below $-50 \mathrm{dBc}$. Based on this number, a system spec of the filter is given in Table [2-5] below:

\begin{tabular}{|l|l|l|}
\hline Center frequency & 73.45 & $\mathrm{GHz}$ \\
\hline Passband & $71-76$ & $\mathrm{GHz}$ \\
\hline FBW & 6.8 & $\%$ \\
\hline In-band Return Loss & $<-15$ & $\mathrm{~dB}$ \\
\hline Insertion Loss & $<5$ & $\mathrm{~dB}$ \\
\hline Out-of-band attenuation & -25 & $\mathrm{~dB} @ 68 \mathrm{GHz} \& 80 \mathrm{GHz}$ \\
\hline
\end{tabular}

Table 2-5 Filter specification

A planar filter designed in the LTCC module at this frequency will suffer from a very high conductor loss and $5 \mathrm{~dB}$ is a normal value. However, if the filter is designed by a substrate integrated waveguide (SIW) structure, the insertion loss can be improved down to $2 \mathrm{~dB}$ or $3 \mathrm{~dB}$ since conductor losses will be reduced.

\subsection{Microwave Component Design}

The design of the planar filter requires some background knowledge of microwave circuit design. In this section, we present a quick view of some structures and some key design equations.

Transmission line structure is one of the most commonly used microwave components due to its simplicity. Figure [2-6] shows a small piece of transmission line model defined at an infinitesimal physical length $\Delta z$. Each lumped element value is also defined on this small piece. Its circuit model can be defined by the famous telegrapher equations: 


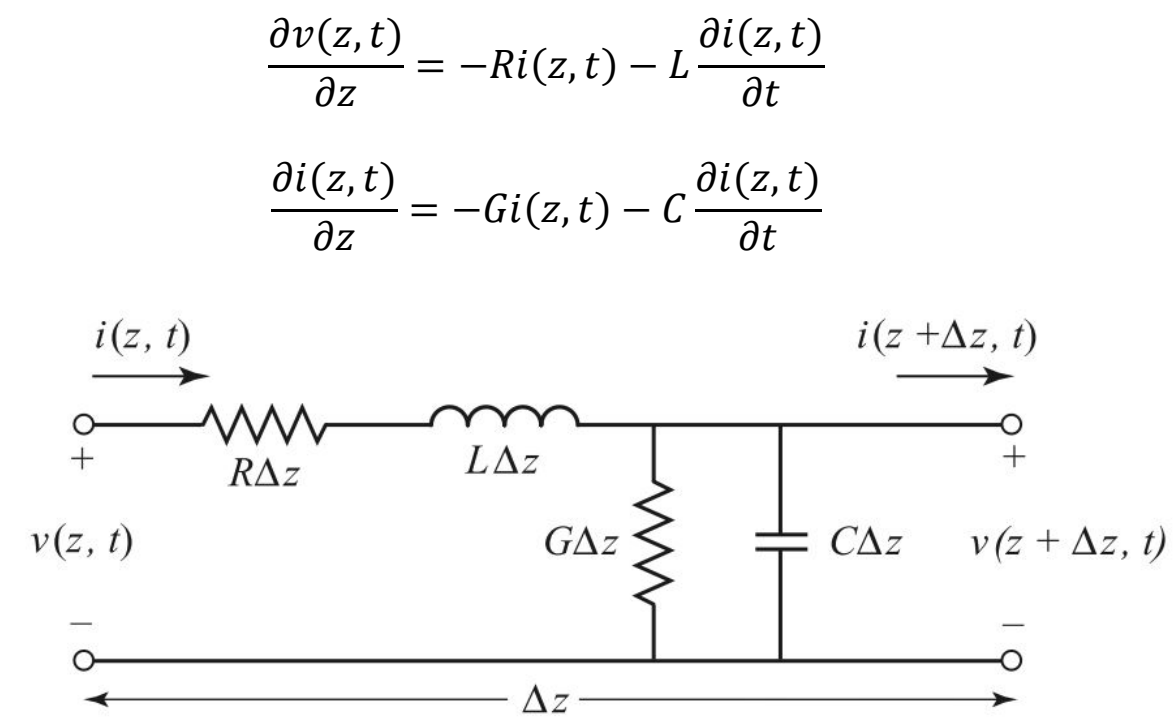

Figure 2-15 Lumped element Transmission Line model

The important design parameter characteristic impedance $Z_{o}$ is defined as:

$$
Z_{o}=\frac{R+j \omega L}{\gamma}=\sqrt{\frac{R+j \omega L}{G+j \omega C}}
$$

Equation (3) is the general definition of characteristic impedance. Figure [2-16] and Figure [2-17] are the most common two planar transmission line structure. The mathematical method for deriving the characteristic impedance is beyond the range.

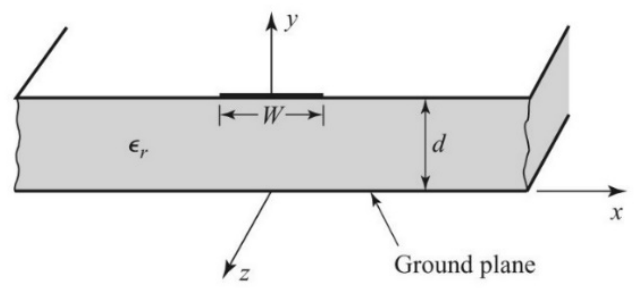

(a)

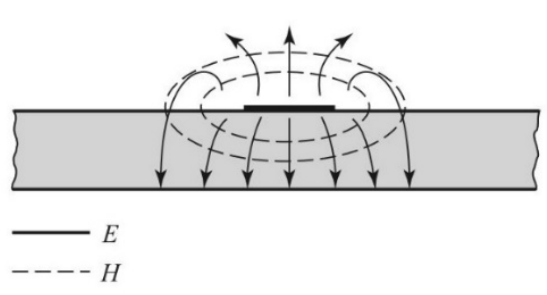

(b)

Figure 2-16 geometry of a microstrip line (b) sketch of the field lines 


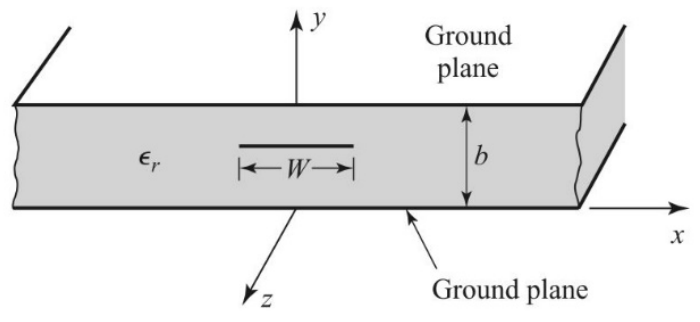

(a)

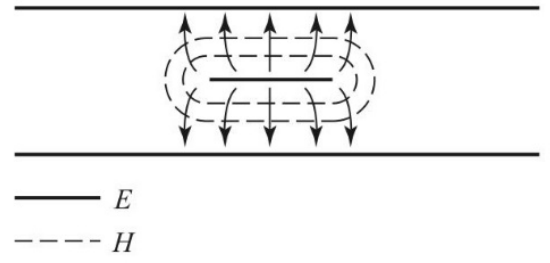

(b)

Figure 2-17 (a) geometry of stripline (b) sketch of the field lines

For a microstrip structure, the relation between characteristic impedance and its width $W$ is[4]:

$$
Z_{0}= \begin{cases}\frac{60}{\sqrt{\epsilon_{e}} \ln \left(\frac{8 d}{W}+\frac{W}{4 d}\right)} & \text { for } \frac{W}{d} \leq 1 \\ \frac{120 \pi}{\sqrt{\epsilon_{e}}\left[\frac{W}{d}+1.393+0.667 \ln \left(\frac{W}{d}+1.444\right)\right]} & \text { for } \frac{W}{d}>1\end{cases}
$$

Where

$$
\epsilon_{e}=\frac{\epsilon_{r}+1}{2}+\frac{\epsilon_{r}-1}{2} \frac{1}{\sqrt{1+12 d / W}}
$$

For most of the time, the characteristic impedance is given, and we want to know the width.

The synthesize equation for design purpose is:

$$
\frac{W}{d}= \begin{cases}\frac{8 e^{A}}{e^{2 A}-2} & \text { for } \frac{W}{d}>2 \\ \frac{2}{\pi}\left[B-1-\ln (2 B-1)+\frac{\epsilon_{r}-1}{2 \epsilon_{r}}\left\{\ln (B-1)+0.39-\frac{0.61}{\epsilon_{r}}\right\}\right] & \text { for } \frac{W}{d}<2\end{cases}
$$

Where:

$$
A=\frac{Z_{0}}{60} \sqrt{\frac{\epsilon_{r}+1}{2}}+\frac{\epsilon_{r}-1}{\epsilon_{r}+1}\left(0.23+\frac{0.11}{\epsilon_{r}}\right)
$$




$$
B=\frac{377 \pi}{2 Z_{0} \sqrt{\epsilon_{r}}}
$$

For a stripline structure in Figure [2-8], the relation between characteristic impedance and conductor width is[4]:

$$
Z_{0}=\frac{30 \pi}{\sqrt{\mu_{r}}} \frac{b}{W_{e}+0.441 b}
$$

Where

$$
\frac{W_{e}}{b}=\frac{W}{b}- \begin{cases}0 & \text { for } \frac{W}{b}>0.35 \\ \left(0.35-\frac{W}{b}\right)^{2} & \text { for } \frac{W}{b}<0.35\end{cases}
$$

The synthesize equation for design purpose is:

$$
\frac{W}{b}= \begin{cases}x & \text { for } \sqrt{\epsilon_{r}} Z_{0}<120 \Omega \\ 0.85-\sqrt{0.6-x} & \text { for } \sqrt{\epsilon_{r}} Z_{0}>120 \Omega\end{cases}
$$

Where

$$
x=\frac{30 \pi}{\sqrt{\epsilon_{r}} Z_{0}}-0.441
$$

\subsection{Resonator design}

A resonator is a basic element for a bandpass filter structure. In planar microwave resonator design, an open or short ended transmission line model can be considered as a series or parallel lumped resonator within a certain frequency range. First, a lumped series resonator model is shown in Figure [2-18]. By network analysis, its input impedance is:

$$
Z_{\text {in }}=R+j \omega L-j \frac{1}{\omega C}
$$

Two important parameters, the center frequency $\omega_{0}$ and quality factor $Q$ is defined as: 


$$
\begin{gathered}
\omega_{0}=\frac{1}{\sqrt{L C}} \\
Q=\frac{\omega_{0} L}{R}=\frac{1}{\omega_{0} R C}
\end{gathered}
$$

Applying mathematic manipulation, and assuming that $\Delta \omega$ is within a small percentage, the input impedance can be rewritten as:

$$
Z_{i n}=R+j \frac{2 R Q \Delta \omega}{\omega_{0}}
$$

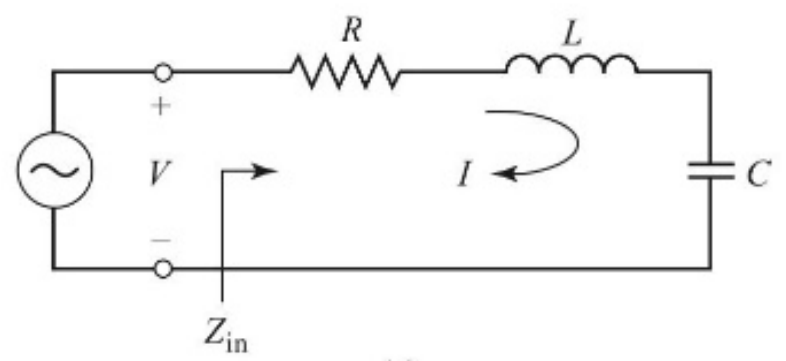

Figure 2-18 Lumped Series Resonator Model

Similarly, for a parallel lumped resonator model in Figure [2-19], its input impedance is given as:

$$
Z_{\text {in }}=\left(\frac{1}{R}+\frac{1}{j \omega L}+j \omega C\right)^{-1}
$$

Equations for center frequency and quality factor:

$$
\begin{aligned}
& \omega_{0}=\frac{1}{\sqrt{L C}} \\
& Q=\omega_{0} R C
\end{aligned}
$$

After simplification, its input impedance can be rewritten as:

$$
Z_{\text {in }} \cong \frac{R}{1+2 j \Delta \omega R C}=\frac{R}{1+2 j Q \Delta \omega / \omega_{0}}
$$




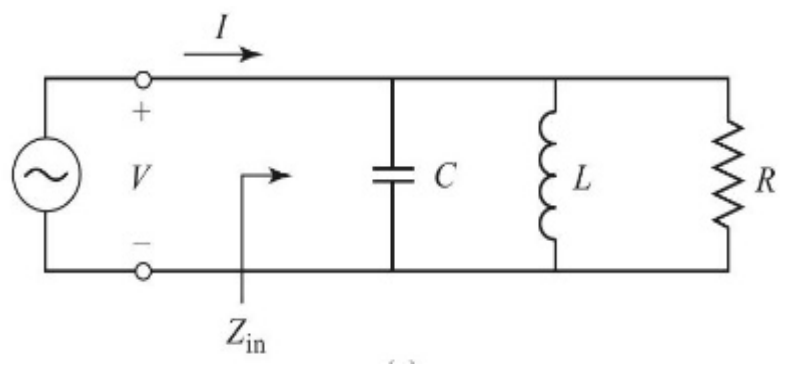

Figure 2-19 Lumped Parallel Resonator Model

Now we investigate the input impedance of a terminated transmission line. Figure [2-20] is a transmission line terminated with an open end, and its input impedance is given by the equation:

$$
\begin{aligned}
& Z_{\text {in }}=Z_{0} \operatorname{coth}(\alpha+j \beta) l=Z_{0} \frac{1+j \tan (\beta l) \cdot \tanh (\alpha l)}{\tanh (\alpha l)+j \tan (\beta l)} \\
& \text {-ーー } \\
& \begin{array}{ccc}
Z_{0}, \beta & V_{L} & Z_{L}=\infty \\
-l & \stackrel{1}{1} & \\
\hline & 0 & z
\end{array}
\end{aligned}
$$

Figure 2-20 Transmission Line Terminated at an open-end

For a narrow band linear assumption:

$$
\begin{gathered}
\tan (\beta l)=\tan \frac{\Delta \omega \pi}{\omega_{0}} \cong \frac{\Delta \omega \pi}{\omega_{0}} \\
\tanh (\alpha l) \cong \alpha l
\end{gathered}
$$

the input impedance in equation (2.2) can be rewritten as:

$$
Z_{\text {in }}=\frac{Z_{0}}{\alpha l+j\left(\frac{\Delta \omega \pi}{\omega}\right)}
$$


Comparing this formula with equation (16), we can see similarities. Therefore, this transmission line with an open-end structure can be modeled as a parallel resonator for a narrow bandwidth assumption where the resistance of the equivalent circuit is:

$$
R=\frac{Z_{0}}{\alpha l}
$$

The capacitance of the equivalent circuit is:

$$
C=\frac{\pi}{2 \omega_{0} Z_{0}}
$$

And the inductance of the equivalent circuit is:

$$
L=\frac{1}{\omega_{0}^{2} C}
$$

Then the quality factor can be rewritten as:

$$
Q=\omega_{0} R C=\frac{\pi}{2 \alpha l}=\frac{\beta}{2 \alpha}
$$

\subsection{Summary}

In this chapter, an E-band RF transceiver structure is present for a potential use in $5 \mathrm{G}$ architecture. Analysis of the RF transceiver up-mixing process shows that the third harmonic and fourth harmonic of Lo signal leakage is $-25 \mathrm{dBc}$. For spur signal attenuation purpose, system specifications for a bandpass filter is proposed. In summary, several key design equations for characterizing transmission line impedance and resonator structure are given. 


\section{Chapter 3: Filter design}

\subsection{General description}

A filter is a two-port network used to control the frequency response at a certain point in an RF or microwave system by providing transmission at frequencies within the passband of the filter and attenuation in the stopband of the filter. Typical frequency responses include low-pass, high-pass, bandpass, and band-reject characteristics. Applications can be found in virtually any type of RF or microwave communication, radar, or test and measurement system.

\subsubsection{Transfer Functions}

An amplitude-squared function for a lossless passive filter network is defined as:

$$
\left|S_{21}(j \Omega)\right|^{2}=\frac{1}{1+\varepsilon^{2} F_{n}^{2}(\Omega)}
$$

Where $\varepsilon$ is the in-band ripple constant. $F_{n}$ represents a filtering or characteristic function, and $\Omega$ represents a radian frequency variable of a lowpass prototype filter that has a cut-off frequency normalized at $\Omega_{\mathrm{c}}=1$

\subsubsection{Poles and Zeros}

For stability purposes, pole and zeros locations of a transfer function must be checked. For a linear time-invariant network, equation (23) can be defined as a rational transfer function:

$$
S_{21}(s)=\frac{N(s)}{D(s)}
$$


This rational transfer function is defined on the $s$ plane where $s=\sigma+j \Omega$. Roots of $N(s)$ are zeros of the filter and roots of $D(s)$ are poles of the filter. These poles are the natural frequencies of the filter whose response is described by $S_{21}(s)$. For the filter to be stable, these natural frequencies must lie in the left half of the p-plane, or on the imaginary axis. If this were not so, the oscillations would be of exponentially increasing magnitude with respect to time, a condition that is impossible in a passive network. The zeros of $N(s)$ are called finite-frequency transmission zeros of the filter.

\subsubsection{Filter Specifications}

For a given transfer function in Eq. (23), the insertion loss response of the filter can be computed by:

$$
L_{A}(\Omega)=10 \log \frac{1}{\left|S_{21}(j \Omega)\right|^{2}} d B
$$

For a lossless passive two-port network, since there is no power dissipation through the network, the return loss response of the filter can be computed by:

$$
L_{R}(\Omega)=10 \log \left[1-\left|S_{21}(j \Omega)\right|^{2}\right] d B
$$

For an ideal filter power response, the in-band insertion loss is $0 \mathrm{~dB}$, and out-of-band return loss is $0 \mathrm{~dB}$, this means that all in-band signal power is transmitted through the network and all out-of-band signal is reflected.

The phase Response based on Eq. (24) can be derived as:

$$
\emptyset_{21}(\Omega)=\operatorname{Arg}_{21}(j \Omega)
$$

The group delay response of this network can then be calculated by: 


$$
\tau_{d}(\Omega)=-\frac{d \emptyset_{21}(\Omega)}{d \Omega} s
$$

Phase delay is the time delay for a steady sinusoidal signal; it represents the time it takes for a carrier signal to travel through the network. The group delay response represents the true signal delay and needs to be considered since a nonlinear group delay means that an early transmitting signal might pass through the network after a later transmitting signal. A flat in-band phase delay is desired for an equal group delay of signals.

\subsection{Filter classes}

The most common three filter classes are Butterworth, Chebyshev, and Elliptic[11]. We first introduce each of their transfer function, discuss its advantage and disadvantage. In the end, another type of filter structure will be presented.

\subsubsection{Butterworth, Chebyshev, and Elliptic}

Butterworth filter is the simplest filter class. It is quite easy to be designed and implemented. But it also suffers from poor selectivity, and sometimes even with a higher-order, it can not meet the required specs. For a Butterworth filter, the characteristic function and the transfer function are defined as:

$$
\begin{gathered}
F_{n}=\Omega^{2 n} \\
\left|S_{21}(j \Omega)\right|^{2}=\frac{1}{1+\Omega^{2 n}}
\end{gathered}
$$

where $\mathrm{n}$ is the degree or the order of the filter, and the $3 \mathrm{~dB}$ cut-off frequency is normalized at $\Omega_{\mathrm{c}}=1$. This structure could provide a maximum in-band flat response. Figure [3-1] 
shows a typical maximally flat frequency response where $L_{A r}$ represents the insertion loss through the network.

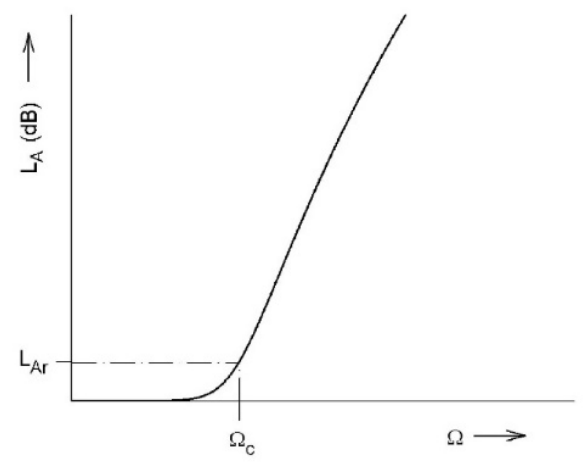

Figure 3-1 Butterworth lowpass prototype frequency response

For design purpose, a plot depicts the relation between the out-of-band attenuation and the filter order is given in Figure [3-2] below.

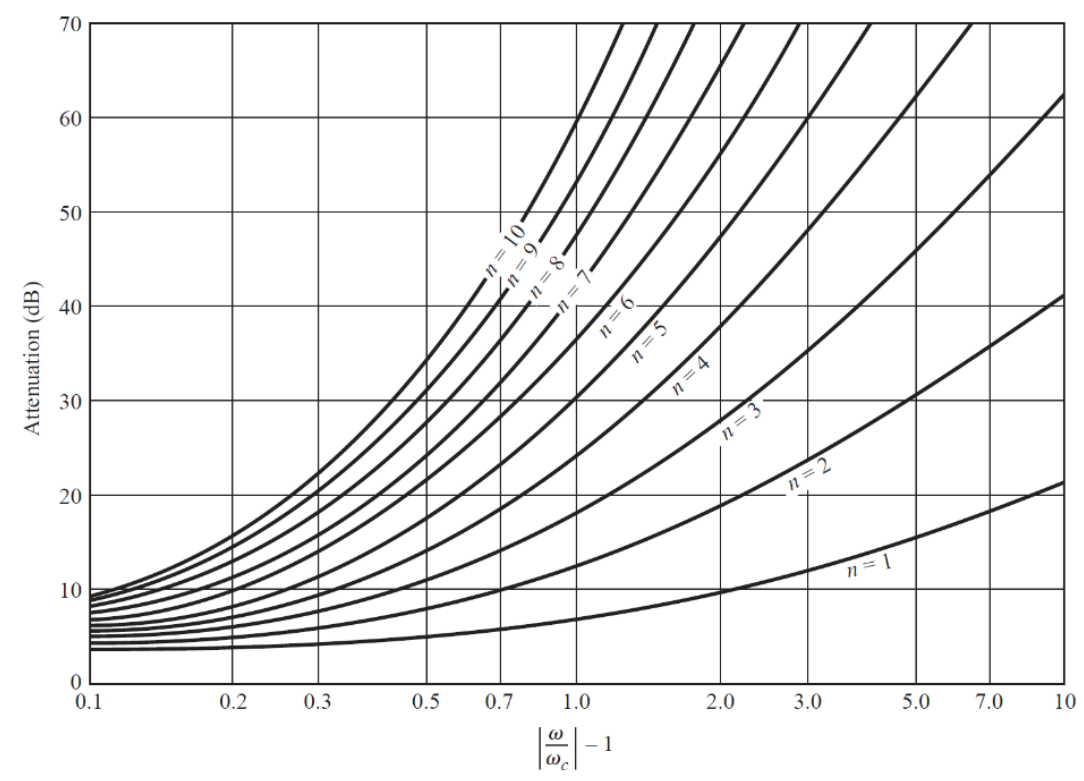

Figure 3-2 Attenuation versus normalized frequency for different order Butterworth filter[11]

To achieve $25 \mathrm{~dB}$ of attenuation at $80 \mathrm{GHz}$ by using a Butterworth filter, a frequency conversion has been down to convert $80 \mathrm{GHz}$ to an offset frequency. This is equivalent to an offset frequency on the higher side: 


$$
\left|\frac{\omega}{\omega_{c}}\right|-1=\frac{80 G H z}{76 \mathrm{~Hz}}-1=1.05-1=0.05
$$

From the figure, to achieve $25 \mathrm{~dB}$ attenuation at 0.05 requires a more than tenth order filter. A tenth order filter indicates ten resonators for a bandpass filter, and the insertion loss due to conductor loss and dissipation loss will be huge.

Figure [3-3] illustrates the frequency response of a Chebyshev lowpass prototype filter. Compared to the Butterworth filter, Chebyshev filter has a better frequency selectivity; its disadvantage is that the in-band frequency response is not flat. For a Chebyshev filter, the in-band ripple level is related to the in-band insertion loss as:

$$
\varepsilon=\sqrt{10^{\frac{L_{A r}}{10}}-1}
$$

Its characteristic function is defined as a Chebyshev function:

$$
F n= \begin{cases}\cos \left(n \cos ^{-1} \Omega\right) & |\Omega| \leq 1 \\ \cosh \left(n \cos ^{-1} \Omega\right) & |\Omega| \geq 1\end{cases}
$$

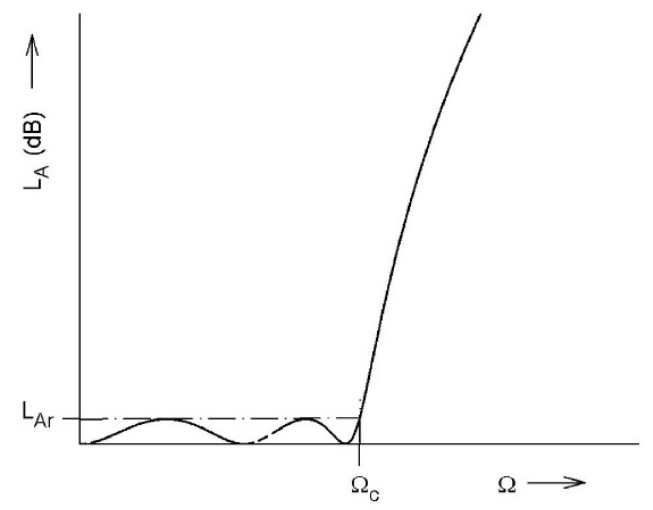

Figure 3-3 Chebyshev lowpass prototype frequency response

The relation between the out-of-band attenuation and filter order of a Butterworth filter is given in Figure [3-4] below. 


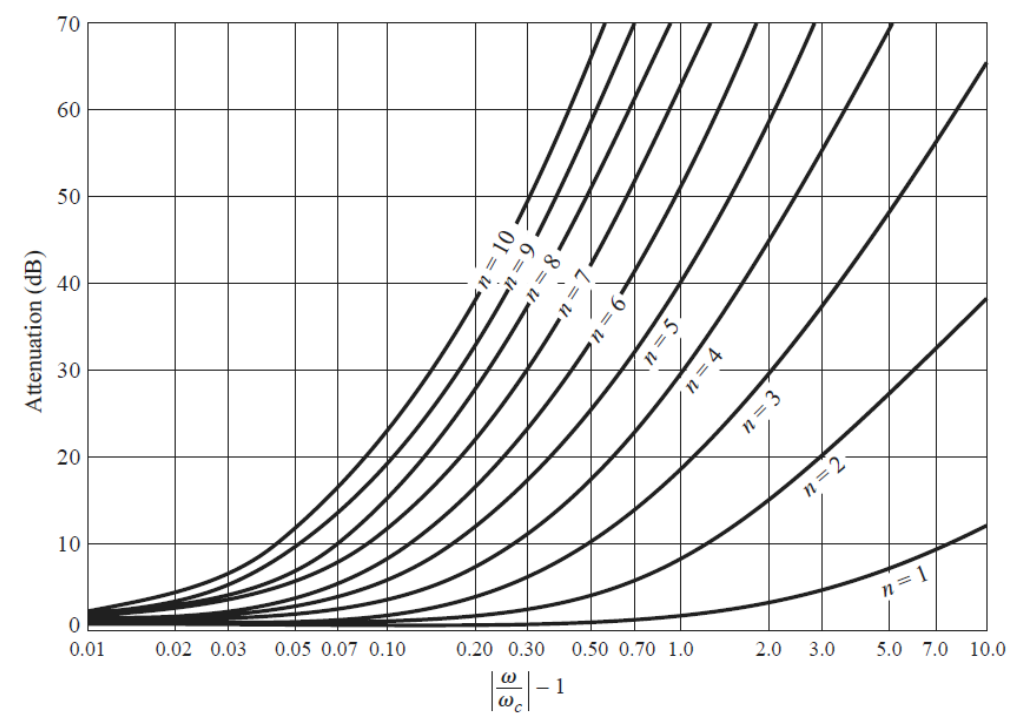

Figure 3-4 Attenuation versus normalized frequency for Chebyshev filter[11]

To achieve $25 \mathrm{~dB}$ attenuation at 0.05 normalized frequency offset, from the plot, the filter order is still more than ten. Still, such a higher-order filter is not a good option, especially in the E-band frequency, because the conductor loss increases as the frequency increases. Figure [3-5] depicts an elliptic filter frequency response. The elliptic filter has a complicated topology, but it could provide a very sharp frequency selectivity. For an Elliptic filter, the characteristic function $F n$ is a complicated elliptic function. It can generate theoretically infinite transmission zeros at out-off-band frequency. Therefore, the frequency selectivity will be improved. Its transfer function shows both an in-band ripple and an out-of-band equal-ripple behavior. 


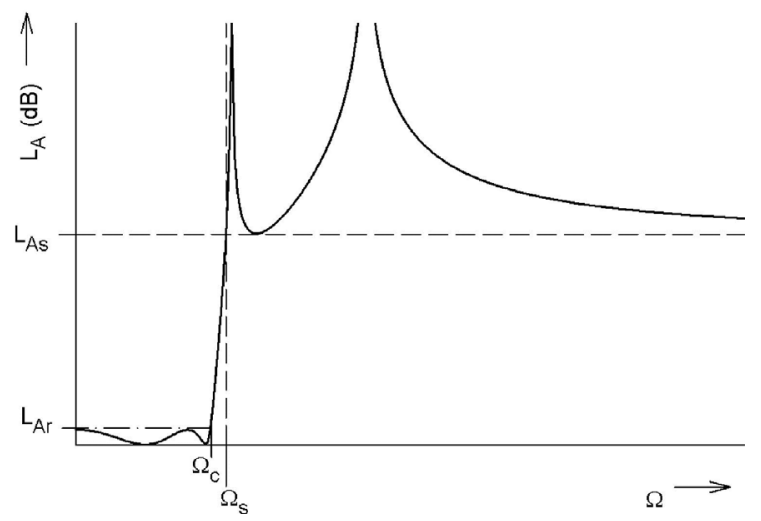

Figure 3-5 Elliptic lowpass prototype frequency response

A lower-order elliptic filter can be enough to meet the filter spec since it creates several near band transmission zeros which can largely improve its frequency selectivity. However, these transmission zeros also increase its design complexity.

\subsubsection{Selective Filters with a Single Pair of Transmission Zero}

In summary, Butterworth and Chebyshev filters are easy to implement, but they cannot provide enough selectivity to meet system specifications. The elliptic filter has a higher selectivity, but it suffers from design complexity.

Another type of filter, quasi-elliptic filter, with a characteristic function as[12]:

$$
F_{n}(\Omega)=\cosh \left\{(n-2) \cosh ^{-1}(\Omega)+\cosh ^{-1}\left(\frac{\Omega_{a} \Omega-1}{\Omega_{a}-\Omega}\right)+\cosh ^{-1}\left(\frac{\Omega_{a} \Omega+1}{\Omega_{a}+\Omega}\right)\right\}
$$

where $\Omega$ is the frequency variable that is normalized to the lowpass prototype filter. $\pm \Omega_{a}$ is the normalized frequency location of the introduced pair of transmission zero on purpose. By intentionally putting this pair of transmission zeros $\pm \Omega_{a}$ at certain finite out-of-band frequencies, this filter provides a much-improved skirt selectivity, making it a viable intermediate case between the Chebyshev and elliptic-function filters, yet with little practical difficulty for physical realization[13]. This filter is also called a quasi-elliptic 40 
filter since it has only one pair of transmission zeros on finite frequency compared with real elliptic filter. Some reference[14] also provides the design of this type of filter with transmission zeros at both real and imaginary frequencies so it can improve both skirt selectivity and flat in-band group delay.

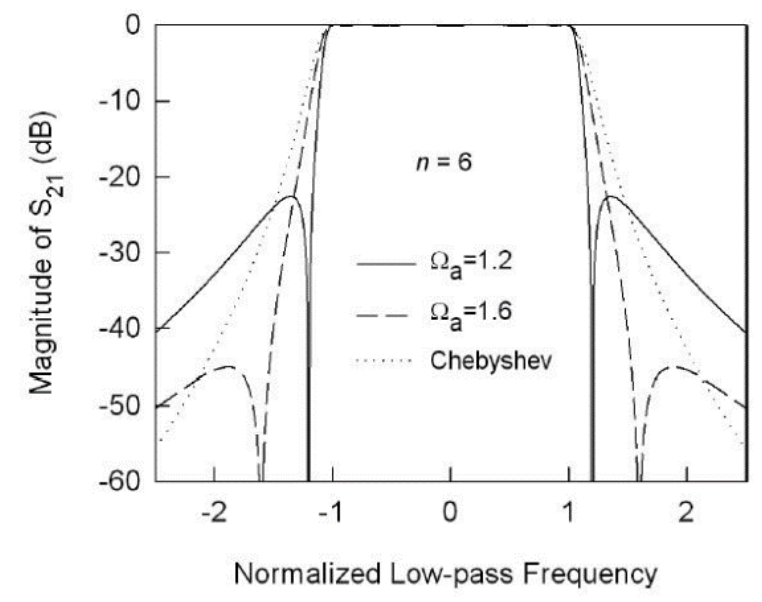

Figure 3-6 Compare with Chebyshev and the filter with a signal pair of attenuation pole at finite frequency.[15]

Figure [3-6] shows some typical frequency response of this type of filters for $n=6$ and $\mathrm{L}_{\mathrm{R}}=-20 \mathrm{~dB}$, with transmission zero at different offset frequency, as compared to that of the same order Chebyshev filter. As can be seen, the improvement in selectivity over the Chebyshev filter is evident. The closer the attenuation poles to the cut-off frequency, the sharper the filter skirt, and the higher the selectivity.

\subsection{Filter Synthesis}

\subsubsection{Lowpass prototype}

A lowpass prototype circuit is a circuit network synthesized from the desired transfer function. It provides the same frequency response as the transfer function mentioned above 41 
provides. Figure [3-7] represents a common lowpass prototype circuit which can be used to design a Butterworth or Chebyshev filter. $g_{1}$ to $g_{n}$ are reactive components which can be implemented by capacitors or inductors at low frequency or by transmission lines at RF frequency.
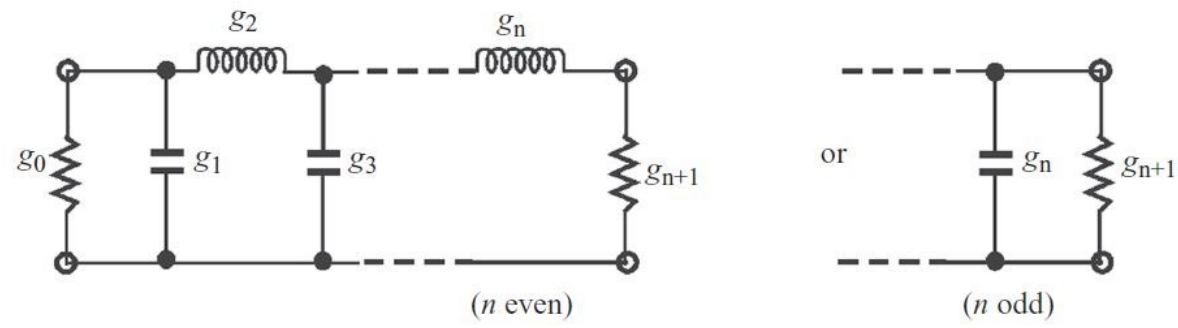

Figure 3-7 Lowpass prototype for Butterworth and Chebyshev filter

Normally, a table will be presented for filter designs. For different order filter, these reactive components' values also differ. Table [3-1] lists element values for a Butterworth prototype with filter order $\mathrm{n}$ from 1 to 9 . Table [3-2] lists element values for a Chebyshev filter with filter order $\mathrm{n}$ from 1 to 9 for an in-band ripple equal to $0.1 \mathrm{~dB}$.

\begin{tabular}{lcccccccccr}
\hline$n$ & $g_{1}$ & \multicolumn{1}{c}{$g_{2}$} & \multicolumn{1}{c}{$g_{3}$} & $g_{4}$ & $g_{5}$ & $g_{6}$ & $g_{7}$ & $g_{8}$ & $g_{9}$ & $g_{10}$ \\
\hline 1 & 2.0000 & 1.0 & & & & & & & & \\
2 & 1.4142 & 1.4142 & 1.0 & & & & & & & \\
3 & 1.0000 & 2.0000 & 1.0000 & 1.0 & & & & & & \\
4 & 0.7654 & 1.8478 & 1.8478 & 0.7654 & 1.0 & & & & & \\
5 & 0.6180 & 1.6180 & 2.0000 & 1.6180 & 0.6180 & 1.0 & & & & \\
6 & 0.5176 & 1.4142 & 1.9318 & 1.9318 & 1.4142 & 0.5176 & 1.0 & & & \\
7 & 0.4450 & 1.2470 & 1.8019 & 2.0000 & 1.8019 & 1.2470 & 0.4450 & 1.0 & & \\
8 & 0.3902 & 1.1111 & 1.6629 & 1.9616 & 1.9616 & 1.6629 & 1.1111 & 0.3902 & 1.0 & \\
9 & 0.3473 & 1.0000 & 1.5321 & 1.8794 & 2.0000 & 1.8794 & 1.5321 & 1.0000 & 0.3473 & 1.0 \\
\hline
\end{tabular}

Table 3-1 Element Values for Butterworth Lowpass Prototype Filters [4] 


\begin{tabular}{lllllllllll}
\multicolumn{1}{l}{ For passband ripple $L_{A r}=0.1 \mathrm{~dB}$} \\
\hline$n$ & $g_{1}$ & $g_{2}$ & $g_{3}$ & $g_{4}$ & $g_{5}$ & $g_{6}$ & $g_{7}$ & $g_{8}$ & $g_{9}$ & $g_{10}$ \\
\hline 1 & 0.3052 & 1.0 & & & & & & & & \\
2 & 0.8431 & 0.6220 & 1.3554 & & & & & & & \\
3 & 1.0316 & 1.1474 & 1.0316 & 1.0 & & & & & & \\
4 & 1.1088 & 1.3062 & 1.7704 & 0.8181 & 1.3554 & & & & & \\
5 & 1.1468 & 1.3712 & 1.9750 & 1.3712 & 1.1468 & 1.0 & & & & \\
6 & 1.1681 & 1.4040 & 2.0562 & 1.5171 & 1.9029 & 0.8618 & 1.3554 & & & \\
7 & 1.1812 & 1.4228 & 2.0967 & 1.5734 & 2.0967 & 1.4228 & 1.1812 & 1.0 & & \\
8 & 1.1898 & 1.4346 & 2.1199 & 1.6010 & 2.1700 & 1.5641 & 1.9445 & 0.8778 & 1.3554 & \\
9 & 1.1957 & 1.4426 & 2.1346 & 1.6167 & 2.2054 & 1.6167 & 2.1346 & 1.4426 & 1.1957 & 1.0 \\
\hline
\end{tabular}

Table 3-2 Element Values for Chebyshev Lowpass Prototype Filters [4]

The filter mentioned in section 3.2.2 with one pair of attenuation poles at a finite frequency can be realized using filters with cross-couplings between nonadjacent resonators[16]. These cross-couplings give several alternative paths which a signal may take between the input and output ports. Figure [3-8] is a lowpass prototype of such a filter with crosscouplings between nonadjacent resonators realized by $J_{m}$ and $J_{m-1}$. Traditionally, the cross-coupled resonator filters are realized using waveguide cavities or dielectric resonator loaded cavities because of their low loss. However, in order to reduce size, weight, and cost, there has been a growing interest in design filters in planar structures[17][18][19].

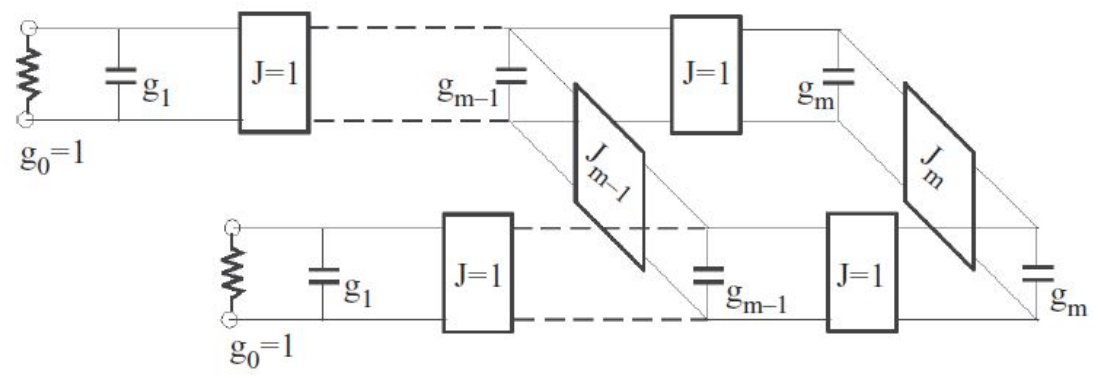

Figure 3-8 Lowpass prototype for quasi-elliptic filter[13]

To find the element values of the low-pass prototype in Figure [3-8], one may use an approximate synthesis method described in [13]. This method is simple, but it suffers from 
inaccuracy and can even fail for highly selective filters that require to move the attenuation poles closer to the cutoff frequencies of the passband. Or we can directly use some design data from [12]. Table [3-3] is the design data for the fourth-order filter, and Table [3-4] is the design data for the sixth order filter.

\begin{tabular}{ccccc}
\hline$\Omega_{a}$ & $g_{1}$ & $g_{2}$ & $J_{1}$ & $J_{2}$ \\
\hline 1.80 & 0.95974 & 1.42192 & -0.21083 & 1.11769 \\
1.85 & 0.95826 & 1.40972 & -0.19685 & 1.10048 \\
1.90 & 0.95691 & 1.39927 & -0.18429 & 1.08548 \\
1.95 & 0.95565 & 1.39025 & -0.17297 & 1.07232 \\
2.00 & 0.95449 & 1.38235 & -0.16271 & 1.06062 \\
2.05 & 0.95341 & 1.37543 & -0.15337 & 1.05022 \\
2.10 & 0.95242 & 1.36934 & -0.14487 & 1.04094 \\
2.15 & 0.95148 & 1.36391 & -0.13707 & 1.03256 \\
2.20 & 0.95063 & 1.35908 & -0.12992 & 1.02499 \\
2.25 & 0.94982 & 1.35473 & -0.12333 & 1.0181 \\
2.30 & 0.94908 & 1.35084 & -0.11726 & 1.01187 \\
2.35 & 0.94837 & 1.3473 & -0.1163 & 1.00613 \\
2.40 & 0.94772 & 1.34408 & -0.10642 & 1.00086 \\
\hline
\end{tabular}

Table 3-3 Element values for Four-Pole Prototype $\left(L_{R}=-20 d B\right)[12]$

\begin{tabular}{lcllcl}
\hline$\Omega_{a}$ & $g_{1}$ & \multicolumn{1}{c}{$g_{2}$} & \multicolumn{1}{c}{$g_{3}$} & \multicolumn{1}{c}{$J_{2}$} & \multicolumn{1}{c}{$J_{3}$} \\
\hline 1.20 & 1.01925 & 1.45186 & 2.47027 & -0.39224 & 1.95202 \\
1.25 & 1.01642 & 1.44777 & 2.30923 & -0.33665 & 1.76097 \\
1.30 & 1.01407 & 1.44419 & 2.21 & -0.29379 & 1.63737 \\
1.35 & 1.01213 & 1.44117 & 2.14383 & -0.25976 & 1.55094 \\
1.40 & 1.01051 & 1.43853 & 2.09713 & -0.23203 & 1.487 \\
1.45 & 1.00913 & 1.43627 & 2.0627 & -0.20901 & 1.43775 \\
1.50 & 1.00795 & 1.4343 & 2.03664 & -0.18962 & 1.39876 \\
1.55 & 1.00695 & 1.43262 & 2.01631 & -0.17308 & 1.36714 \\
1.60 & 1.00606 & 1.43112 & 2.00021 & -0.15883 & 1.34103 \\
\hline
\end{tabular}

Table 3-4 Element values for Six-Pole Prototype $\left(L_{R}=-20 d B\right)[12]$

\subsubsection{Frequency Transformation from Lowpass to Bandpass}

Frequency transformation allows us to transform a filter from its lowpass prototype at frequency domain $\Omega$ to the real frequency domain $\omega$ where a practical filter response is expressed.

44 
Through frequency transformation, a lowpass filter prototype with normalized frequency $\Omega$ can be transformed to the desired bandpass filter centered at $\omega_{0}$ by equation:

$$
\Omega=\frac{1}{F B W}\left(\frac{\omega}{\omega_{0}}-\frac{\omega_{0}}{\omega}\right)
$$

in which $\omega$ is the frequency variable of the bandpass filter, and $F B W$ is the fractional bandwidth of the bandpass filter. $F B W$ and $\omega_{0}$ can be defined by the equation:

$$
\begin{gathered}
F B W=\frac{\omega_{2}-\omega_{1}}{\omega_{0}} \\
\omega_{0}=\sqrt{\omega_{1} \omega_{2}}
\end{gathered}
$$

$\omega_{1}$ and $\omega_{2}$ are the lower and higher edge of the bandpass filter.

For the quasi-elliptic filter lowpass prototype, the transmission zero $\pm \Omega_{a}$ are mapped to two real frequency zeros by:

$$
\begin{gathered}
\omega_{a 1}=\omega_{0} \frac{-\Omega_{a} F B W+\sqrt{\left(\Omega_{a} F B W\right)^{2}+4}}{2} \\
\omega_{a 2}=\omega_{0} \frac{\Omega_{a} F B W+\sqrt{\left(\Omega_{a} F B W\right)^{2}+4}}{2}
\end{gathered}
$$

The inductive/capacitive element $\mathrm{g}$ in the lowpass prototype will transform to a series/parallel LC resonant circuit in the bandpass filter. The elements for the series LC resonator in the bandpass filter are:

$$
\begin{gathered}
L_{s}=\left(\frac{\Omega_{c}}{F B W \omega_{0}}\right) Z_{0} g \\
C_{s}=\left(\frac{F B W}{\omega_{0} \Omega_{c}}\right) \frac{1}{Z_{0} g}
\end{gathered}
$$

And the elements for the parallel LC resonator in the bandpass filter are: 


$$
\begin{gathered}
C_{p}=\left(\frac{\Omega_{c}}{F B W \omega_{0}}\right) \frac{g}{Z_{0}} \\
L_{p}=\left(\frac{F B W}{\omega_{0} \Omega_{c}}\right) \frac{Z_{0}}{g}
\end{gathered}
$$

The element transformation is shown in Figure [3-9]. Each reactive component is converted to a resonator through bandpass transformation. According to equation (18) in chapter 2 , a series resonator can be replaced by a half-wavelength short-end transmission line and a parallel resonator can be replaced by a half-wavelength open-end transmission line.

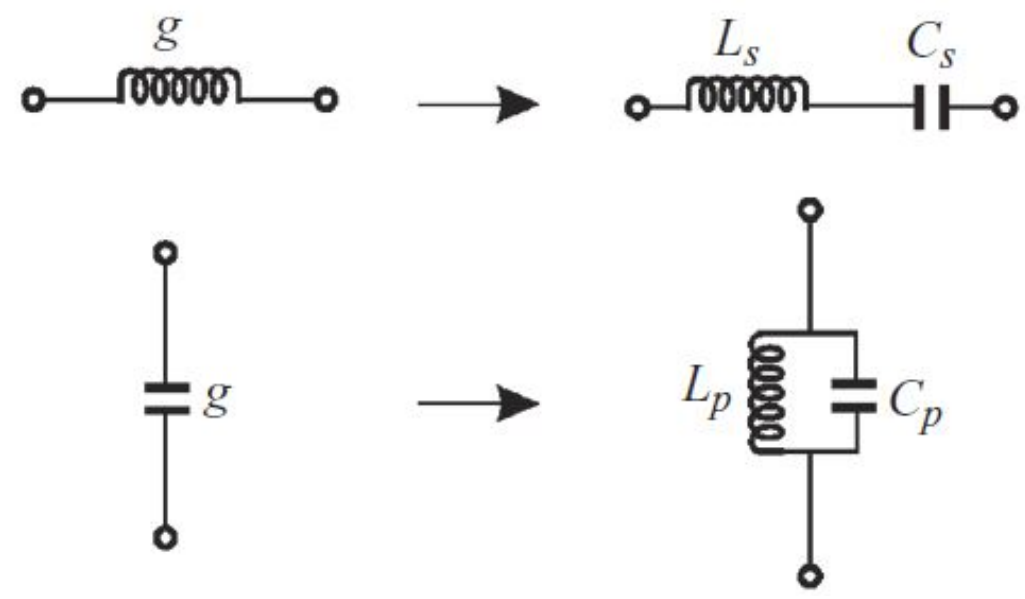

Figure 3-9 Lowpass prototype to bandpass transformation

\subsubsection{Coupling theory for filter design}

Coupled-resonator circuits are of importance for the design of $\mathrm{RF} / \mathrm{microwave}$ filters. It is a very simple technique for designing coupled-resonator filters, and it can be applied to any type of resonator despite its physical structure. It can be used to design waveguide filters, dielectric resonator filters, microstrip filters, and so on. This design method is based on coupling coefficients of each two nearby resonators and the external quality factors of 
the input and output resonators. It is extremely useful for the narrow-band bandpass filter design.

Figure [3-10] shows an equivalent circuit of a coupled-resonator circuit. It can represent most types of bandpass filters. The coupling coefficient between the $n$ and $m$ parallel resonator is represented by $M_{n m}$.

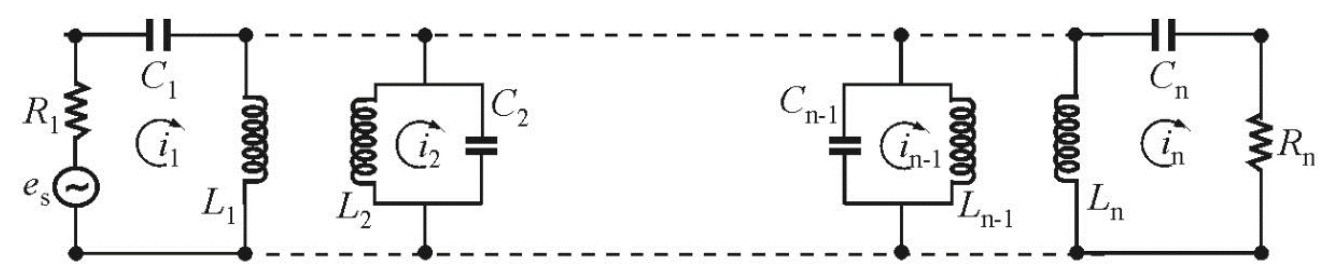

Figure 3-10 Equivalent circuits of n-coupled resonators circuit

The relation between its loop current and voltage is described by a general coupling matrix [A] [15].

$$
[A]=\left[\begin{array}{cccc}
\frac{1}{Q_{e i} \cdot F B W}+p & -\frac{j M_{12}}{F B W} & \cdots & -\frac{j M_{1 n}}{F B W} \\
-\frac{j M_{21}}{F B W} & p & \cdots & -\frac{j M_{2 n}}{F B W} \\
\vdots & \vdots & \vdots & \vdots \\
-\frac{j M_{n 1}}{F B W} & -\frac{j M_{n 1}}{F B W} & \cdots & \frac{1}{Q_{e n} \cdot F B W}+p
\end{array}\right]
$$

Where:

$$
\begin{gathered}
\mathrm{p}=\mathrm{j} \frac{1}{F B W}\left(\frac{\omega}{\omega_{0}}-\frac{\omega_{0}}{\omega}\right) \\
\frac{R}{\omega_{0} L}=\frac{1}{Q_{e i}} \\
M_{i j}=\frac{L_{i j}}{L}
\end{gathered}
$$


For any type of bandpass filter, this general coupling matrix can be derived, and it can fully describe the filter performance. Also, each parameter in the coupling matrix can be obtained from the filter's lowpass prototype parameters.

For Butterworth and Chebyshev lowpass prototypes, the relation between the coupling matrix and the lowpass prototype components are:

$$
\begin{gathered}
Q_{e i}=\frac{g_{0} g_{1}}{F B W} \\
Q_{e n}=\frac{g_{n} g_{n+1}}{F B W} \\
M_{i, i+1}=\frac{F B W}{\sqrt{g_{i} g_{i+1}}}
\end{gathered}
$$

$Q_{e i}$ and $Q_{e n}$ are called external quality factors. It represents the coupling strength between the circuit network and I/O ports. $M_{i, i+1}$ represents the coupling coefficient between every two resonators. For a quasi-elliptic filter, the relationship of the coupling matrix and a lowpass prototype is given by:

$$
\begin{gathered}
Q_{e 1}=Q_{e n}=\frac{g_{1}}{F B W} \\
M_{i, i+1}=M_{n-i, n-i+1}=\frac{F B W}{\sqrt{g_{i} g_{i+1}}} \text { for } i=1 \text { to } m-1, m=\frac{n}{2} \\
M_{m, m+1}=\frac{F B W * J_{m}}{g_{m}} \\
M_{m-1, m+2}=\frac{F B W * J_{m-1}}{g_{m-1}}
\end{gathered}
$$

A general coupling structure for the quasi-elliptic filter is depicted in Figure [3-11], where each node represents a resonator in Figure [3-10], the full lines indicate the main path 
couplings, and the broken line denotes the cross-coupling. It is essential that the phase of cross-coupling $M_{m-1, m+2}$ has a 180 -degree shift to the phase of $M_{m, m+1}$ to achieve a pair of attenuation poles at finite frequencies, where $m=N / 2$ with $N$ being the degree of the filter. This can be achieved by electric coupling on one line and magnetic coupling on another line. Both the electric coupling and magnetic coupling can be simulated by the fullwave electromagnetic (EM) simulation.

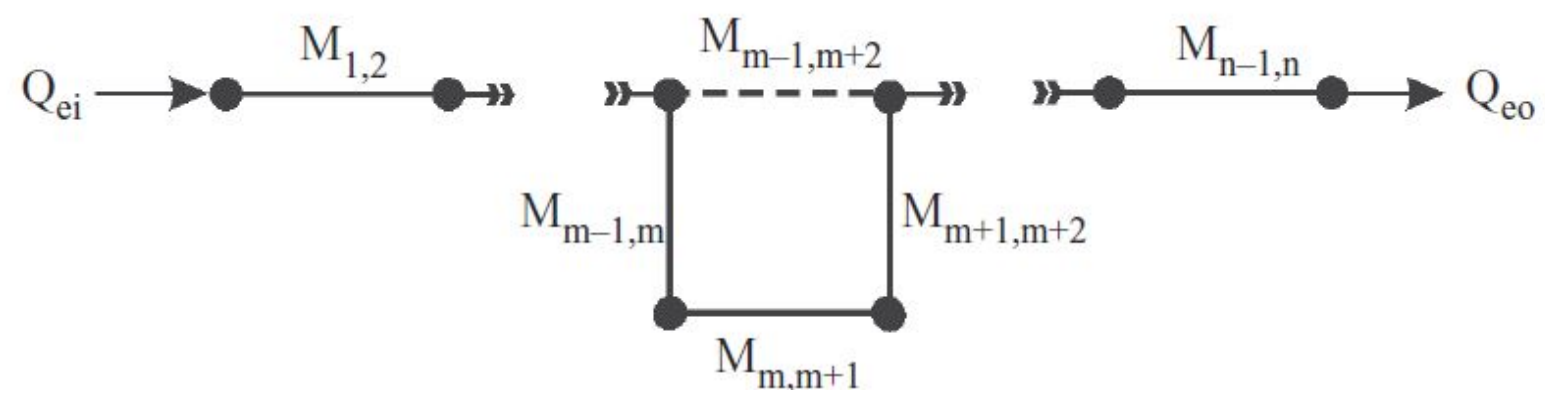

Figure 3-11 General Coupling structure for a bandpass filter with a pair of transmission zero The advantage of using coupling theory for narrow bandwidth bandpass filter design is that coupling coefficient between every two resonators and external quality factors between the port with the filter structure are directly related to each resonators' physical dimension and tapped line position. Those parameters can be easily derived from an EM simulation result.

\subsection{Summary}

Millimeter wave band 5G communication systems require high-performance narrow-band bandpass filters having low insertion loss and high selectivity together with linear phase or flat group delay in the passband. In this chapter, some generic filter types are present, and their advantages and disadvantages are analyzed. The coupling theory method for bandpass 
filter design is also presented. The next chapter will present filter designs that are constructed by this method. 


\section{Chapter 4: Filter Implementation}

To realize a quasi-elliptic filter in the LTCC module, according to [12], a fourth-order filter configuration and a six order filter configuration are presented in Figure [4-1] and [4-2]. In Figure [4-1], the cross-coupling between R1 and R4 is the electric coupling, and the coupling between R2 and R3 is the magnetic coupling. In Figure [4-2], the cross-coupling between R2 and R5 is the magnetic coupling, and the coupling between R3 and R4 is the electric coupling. The explanation of different coupling is discussed in section 4.3.

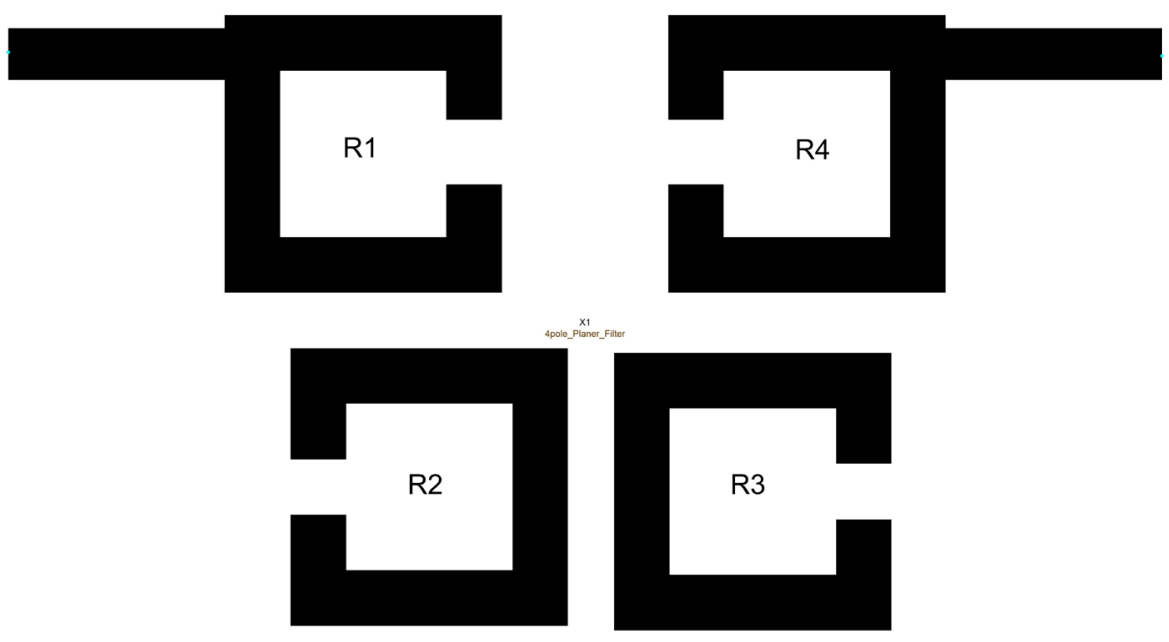

Figure 4-1 Four pole filter

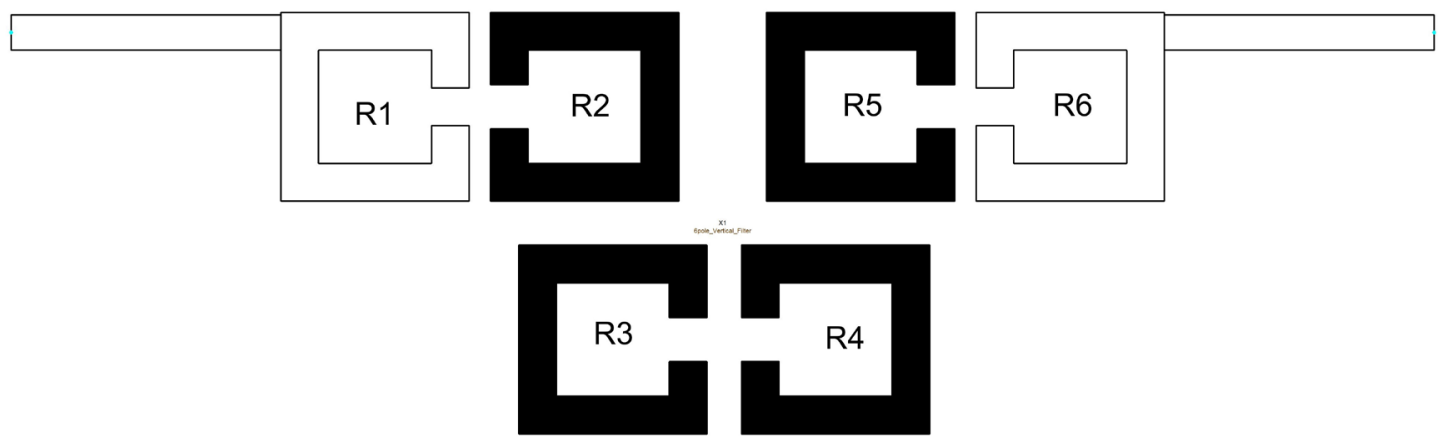

Figure 4-2 six-pole filter structure 
Design parameters for these two filters can be derived from system specs from Table [25]. Based on these specs, a quasi-elliptic lowpass prototype is first chosen from Table [33] and Table [3-4]. Here a four-pole lowpass prototype with transmission zero $\Omega_{a}=1.8$ and a six-pole lowpass prototype with transmission zero $\Omega_{a}=1.2$ are selected. Equation (50)-(53) convert these lowpass prototype parameters into external quality factors and coupling coefficients.

Design parameters for four pole filter:

$$
\begin{gathered}
M_{1,2}=M_{3,4}=0.06 \\
M_{2,3}=0.055 \\
M_{1,4=}-0.015 \\
Q_{e i}=Q_{e o}=13
\end{gathered}
$$

Design parameters for six pole filter:

$$
\begin{gathered}
M_{1,2}=M_{5,6}=0.058 \\
M_{2,3}=M_{4,5}=0.041 \\
M_{3,4}=0.047 \\
M_{2,5}=-.0 .007 \\
Q_{e i}=Q_{e o}=14
\end{gathered}
$$

\subsection{Resonator Design}

A half-wavelength long open-end transmission line is used for a parallel resonator design. The resonator is implemented by a stripline structure in a four-layer LTCC substrate shown in Figure [4-3], which has a relative dielectric constant of 5.8 and a total thickness of 200um. 
The conductor metal thickness is 10um. Figure [4-4] shows a folded half-wavelength square loop.

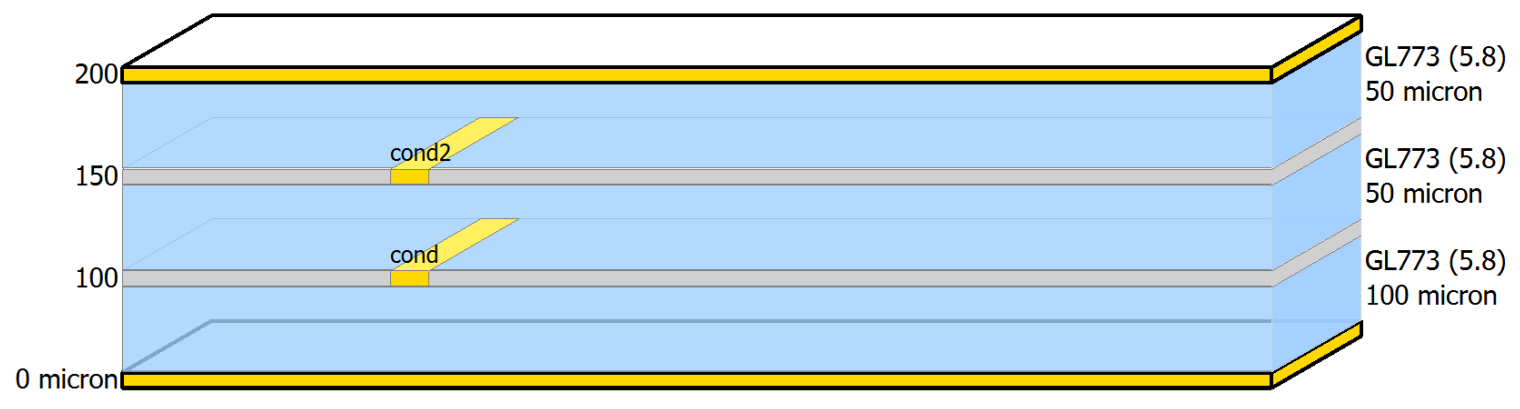

Figure 4-3 LTCC substrate structure

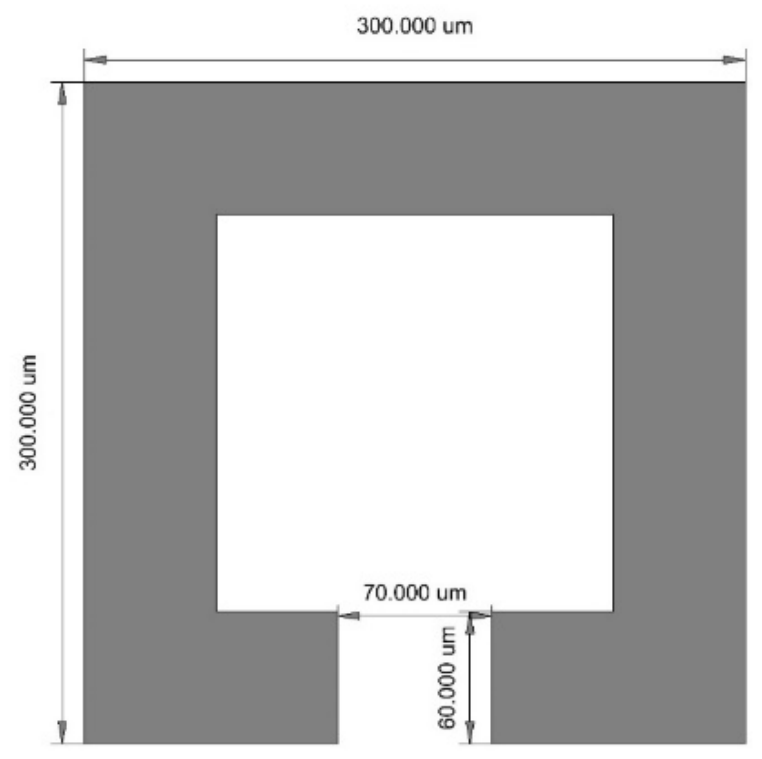

Figure 4-4 folded half-wavelength resonator

\subsection{Extract External Quality Factor}

To characterize the external quality factor in terms of the physical structure, a typical input/output tapped-line coupling structure is built up in Figure [4-5]. In this structure, a $50-\Omega$ feed line is directly tapped on to the I/O resonator, and the external quality factor is 
controlled by the tapping position T. As the distance gets shorter, the tapped line is closer to the virtual ground of the resonator.

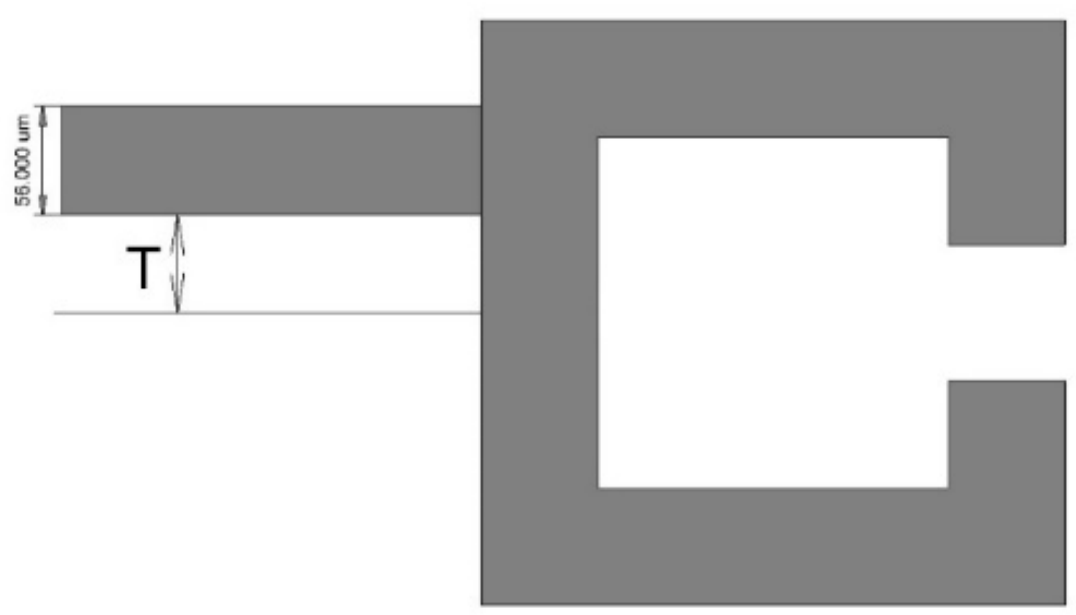

Figure 4-5 tapped-line coupling structure

By performing a network analysis and making a narrow band approximation, the relation between external quality factor and return loss $S_{11}$ can be derived[15]:

$$
S_{11}=\frac{1-j Q_{e} \cdot\left(2 \Delta \omega / \omega_{0}\right)}{1+j Q_{e} \cdot\left(2 \Delta \omega / \omega_{0}\right)}
$$

Assume that the resonator is lossless, the phase response of $S_{11}$ vary with frequency. Figure [4-6(a)] shows that the phase response of $S_{11}$ is a function of $\Delta \omega / \omega_{0}$. When the phase is $\pm 90^{\circ}$ the corresponding value of $\Delta \omega$ is found to be:

$$
2 Q_{e} \frac{\Delta \omega_{ \pm}}{\omega_{o}}=\mp 1
$$

And the external quality factor can be extracted from the relation:

$$
\mathrm{Q}_{\mathrm{e}}=\frac{\omega_{0}}{\Delta \omega_{ \pm 90^{\circ}}}
$$

By adjusting the tapped-line position and measuring each phase response, the relation between external quality factor and tapped line position is depicted in Figure [4-6(b)]. 54 

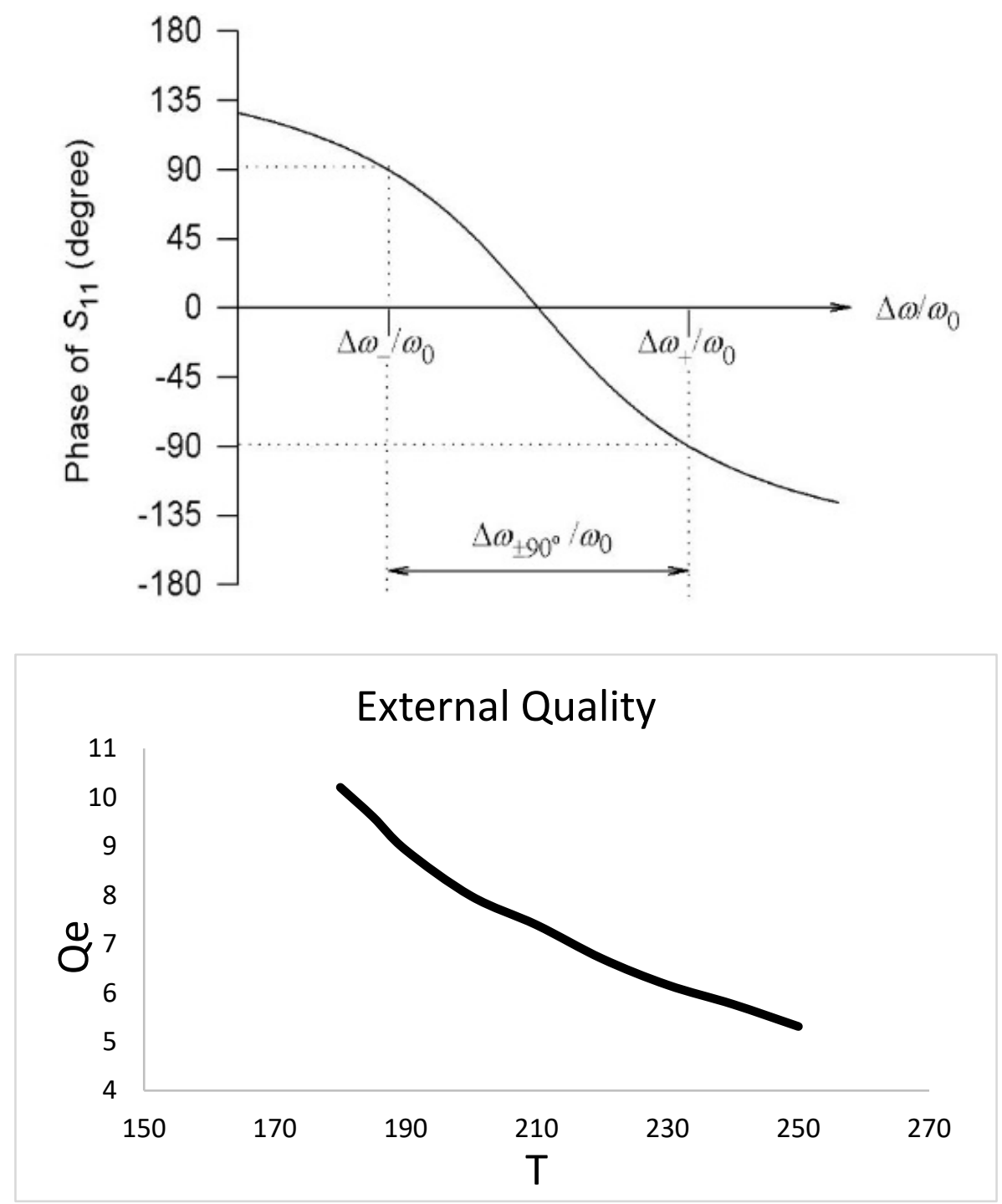

Figure 4-6 (a) phase response of $S_{11}$ (b) External Q

\subsection{Extraction of the Coupling Coefficient}

The next step is to derive coupling coefficients in terms of physical structures. There are three basic coupled structures based on different orientations of a pair of open-loop resonators. The coupling in those structures is achieved through proximity coupling. The nature and the extent of the fringe fields determine the nature and the strength of the 
coupling effect. Because the fringe field exhibits an exponentially decaying character outside the region, the electric fringe fields are stronger near the side with maximum electric field distribution, whereas the magnetic fringe field is stronger near the side having the maximum magnetic field distribution[15]. The coupling coefficient can be extracted from its frequency response through an EM simulation.

\subsubsection{Electrical Coupling}

The electric coupling can be obtained by placing the open sides of two coupled resonators closely, as in Figure [4-7]. The coupling can be purely planar, or it can be both planar and vertical. The vertical coupling can be physically achieved by putting one resonator on the cond2 layer and another one on the cond layer in Figure [4-3].

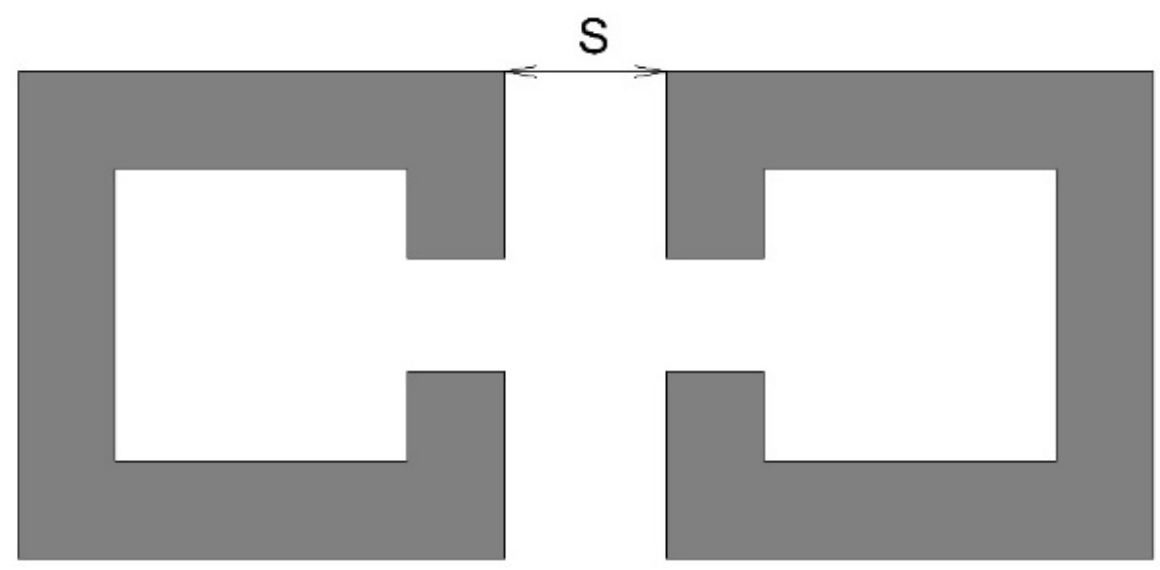

Figure 4-7 Electrical couplings

Figure[4-8(a)] shows the frequency responses of this coupled resonator structure. Here, due to the coupling effect, two natural resonant frequencies will appear. And it has been proved [20] that the two frequencies shown in Figure [4-8(a)] are the two natural resonant frequencies. The coupling coefficient can be extracted through the equation[20]: 


$$
k_{E}=\frac{f_{m}^{2}-f_{e}^{2}}{f_{m}^{2}+f_{e}^{2}}
$$

By varying the distance $S$ between two resonators in Figure [4-7], the relation between coupling coefficient and distance is plotted on Figure [4-8(b)]. Normally, the stronger the coupling, the wider the separation of the two resonant peaks and the deeper the trough in the middle.
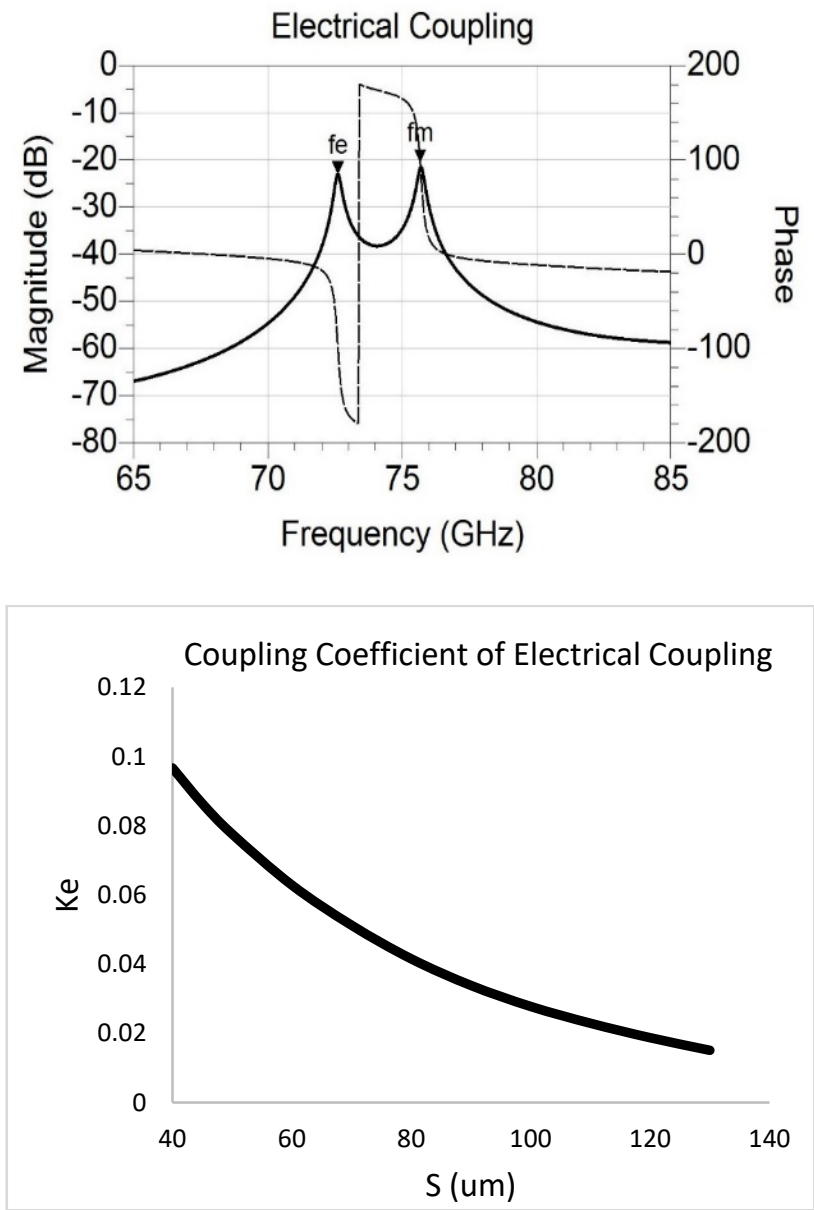

Figure 4-8 (a) frequency response; (b) Coupling Coefficient 


\subsubsection{Magnetic Coupling}

Like the electric coupling, the magnetic coupling can also be obtained by placing the maximum magnetic field sides of two coupled resonators closely, as shown in Figure [49]. The equation used to extract the coupling coefficient is:

$$
k_{M}=\frac{f_{e}^{2}-f_{m}^{2}}{f_{e}^{2}+f_{m}^{2}}
$$

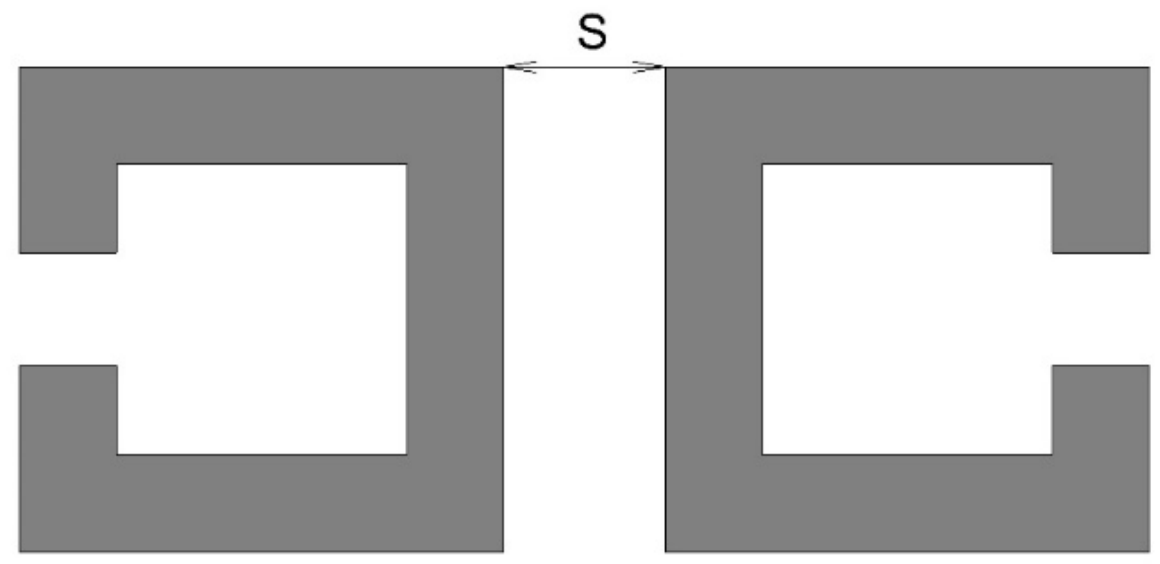

Figure 4-9 Magnetic Coupling

Figure[4-10(a)] is the frequency response of this coupled resonator structure, and the relation between coupling coefficient and distance is extracted in Figure [4-10(b)]. By comparing the phase responses of Figures [4-8(a)] and Figure [4-10(a)], it can be observed that these two structures phase response in Figure [4-8(a)] and Figure [4-10(a)] are indeed out of phase. 

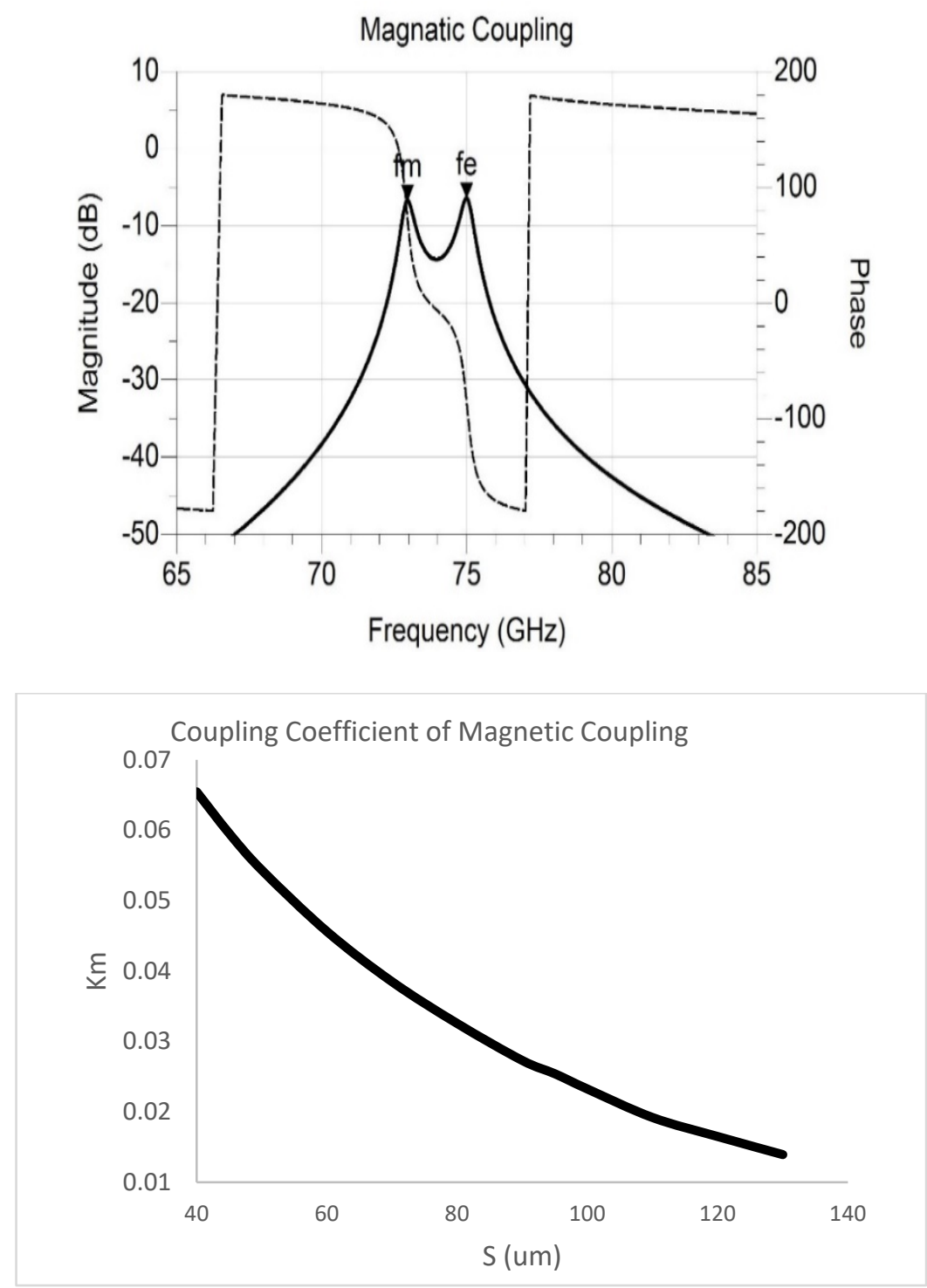

Figure 4-10 (a) Frequency Response of Magnetic Coupling ; (b) Coupling Coefficient versus distance

\subsubsection{Mixed Coupling}

Another type of coupling can be obtained by placing two resonator structures, as shown in Figure [4-11]. In this type of structure, the electric and magnetic fringe fields at the coupled sides may both have comparable distributions, so that both electric and magnetic couplings occur. In this case, the coupling may be referred to as a mixed coupling. 


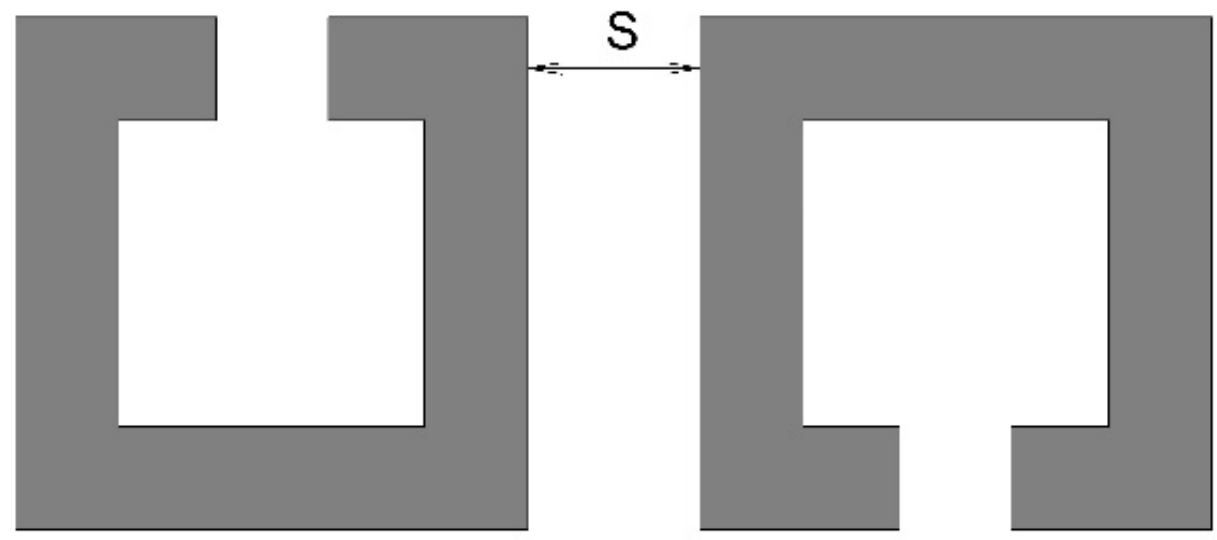

Figure 4-11 Mixed Coupling

The relation between coupling coefficient and distance is shown in Figure [4-12]

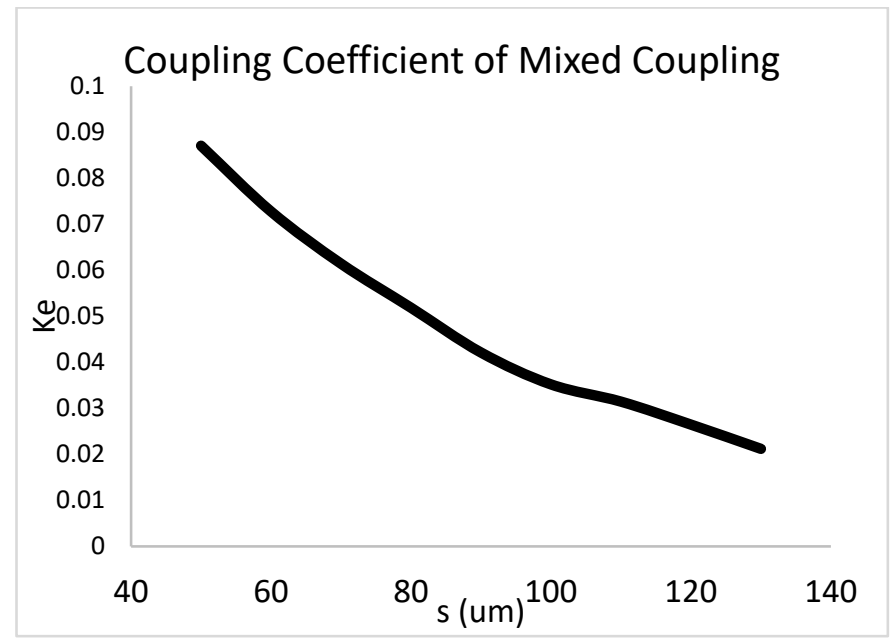

Figure 4-12 The coupling coefficient of Mix Coupling

\subsection{Filter design and simulation}

Figure [4-8(b)], Figure [4-10(b)] and Figure [4-12] provide the relation between physical dimensions of the filter with external quality factor and coupling coefficients. Based on equation (50)-(53), a fourth-order filter is implemented and simulated by ADS[21] in Figure [4-13] 


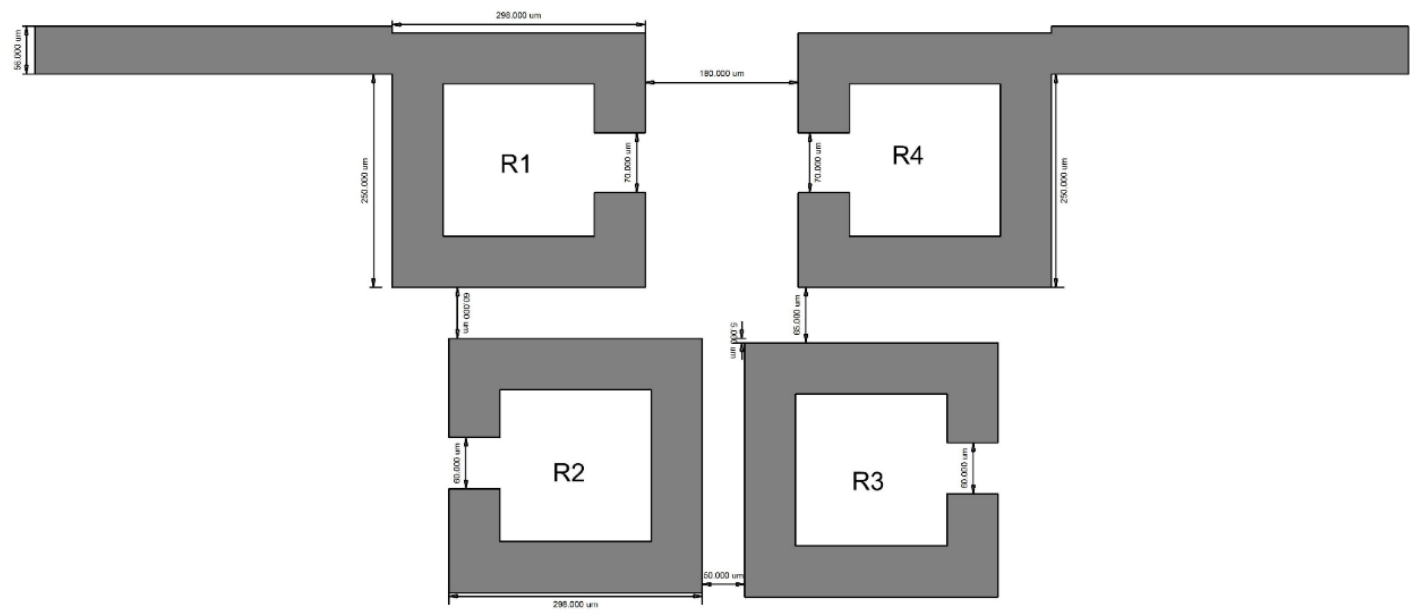

Figure 4-13 Four pole filter

Simulation results are shown in Figure [4-14]. The mid-band insertion loss is about 4.5dB, which is mainly due to the conductor loss of the copper. The two attenuation zeros near the cutoff frequencies of the passband can clearly be identified, one at $66.9 \mathrm{GHz}$ and one at $79.2 \mathrm{GHz}$. The size of the filter is about $780 \mathrm{um}$ by $660 \mathrm{um}$.

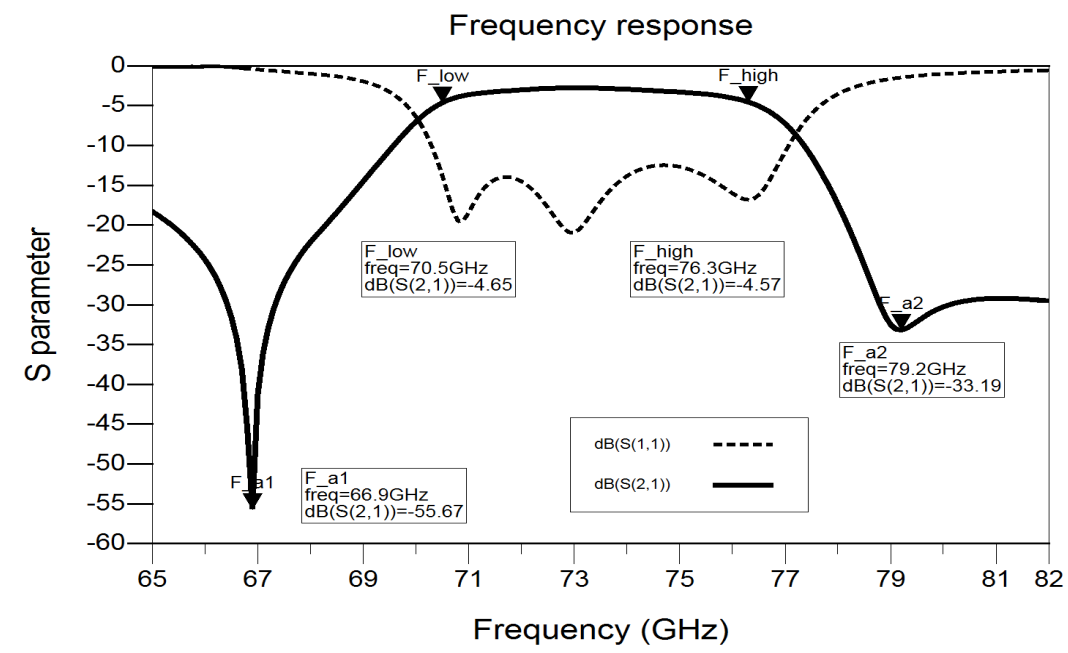

Figure 4-14 Frequency response of Four Pole Filter 
Figure [4-15] is the six-pole filter structure. Its structure is slightly different compared to the four-pole filter. It uses two conductor layers. Resonator R1 and R6 are on upper Layer Cond2, and R2, R3, R4, R5 are on the lower Cond layer. The reason for this arrangement is that the coupling coefficient M12 and M56 requires a quite small physical distance between R1 with R2 and R5 with R6. Due to the fabrication limitation, this distance cannot be satisfied on the same layer. By putting R1 and R6 on the upper conductor layer, this restriction is removed, and the desired coupling coefficient can be obtained.

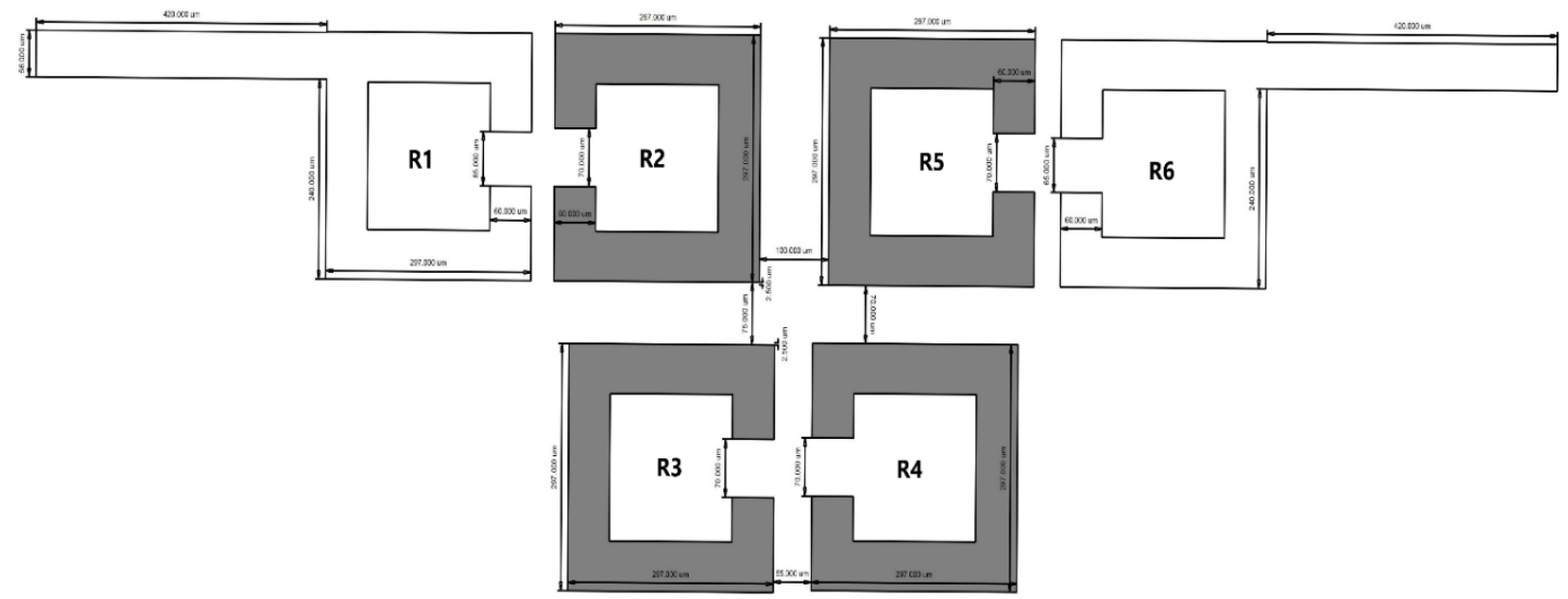

Figure 4-15 six-pole filter structure

Figure [4-16] is the simulation result of this six-pole filter. Compared with Figure [4-14], the $-5 \mathrm{~dB}$ bandwidth ranges from $71.5 \mathrm{GHz}$ to $75.88 \mathrm{GHz}$. This bandwidth is smaller than the four-pole filter bandwidth, but the selectivity is greatly improved. In-band return loss is tuned to be lower than $-10 \mathrm{~dB}$. Two attenuation zeros are located at $68.4 \mathrm{GHz}$ and at 79.8GHz separately. 


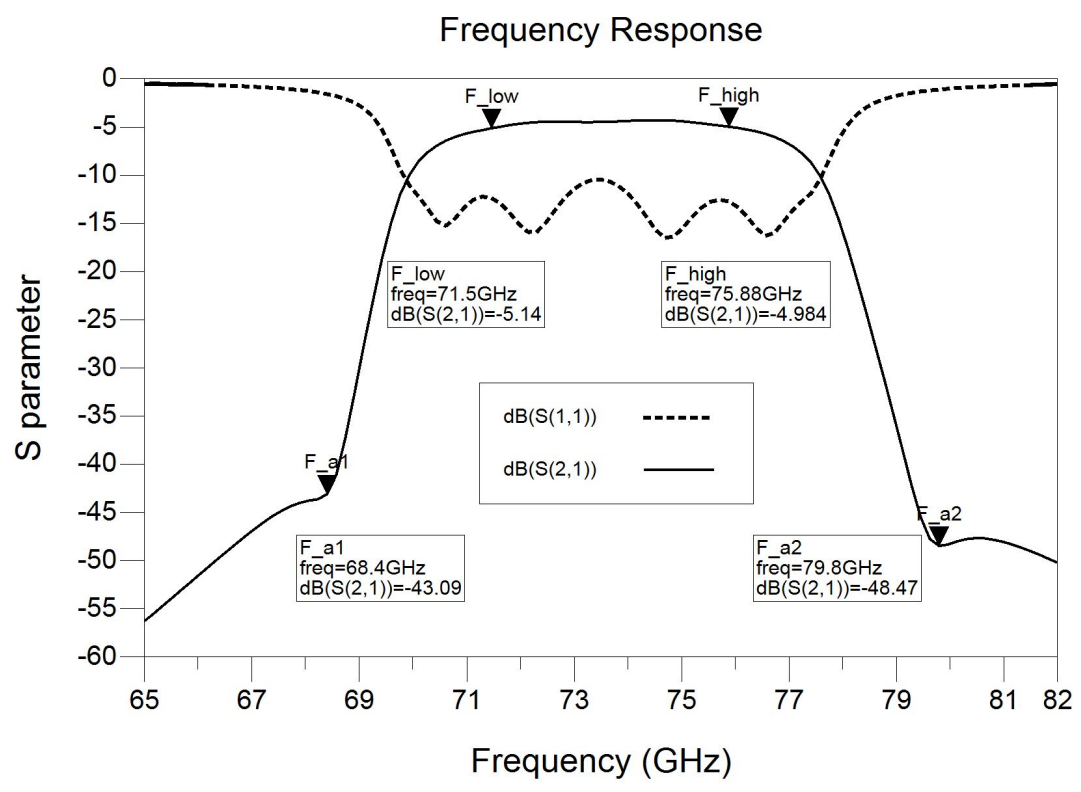

Figure 4-16 Frequency response of Six Pole Filter

Table [4-1] is a comparison of both filter performance with system spec. It indicates that both filters can satisfy the system specifications listed in Chapter 2. The six-pole filter has a better frequency selectivity but suffers from a higher insertion loss. Some further tuning is required to improve their impedance matching.

\begin{tabular}{|l|l|l|l|l|}
\hline & Bandwidth & Insertion loss & Return loss & Attenuation@68\&80GHz \\
\hline System spec & $71-76 \mathrm{GHz}$ & $<5 \mathrm{~dB}$ & $-15 \mathrm{~dB}$ & $>25 \mathrm{~dB}$ \\
\hline Four pole filter & $70.5-76.3 \mathrm{GHz}$ & $4.5 \mathrm{~dB}$ & $<-15 \mathrm{~dB}$ & $>25 \mathrm{~dB}$ \\
\hline Six pole filter & $71.5-75.88 \mathrm{GHz}$ & $5 \mathrm{~dB}$ & $<-10 \mathrm{~dB}$ & $>45 \mathrm{~dB}$ \\
\hline
\end{tabular}

Table 4-1 Comparison of system spec with two filter performance

\subsection{Monte Carlo Analysis}

Monte Carlo Analysis is a method for testing the sensitivity of the filter. For fabrication purpose, the filter must be insensitive to the design variation of design parameters such as metal width, resonator length, and the distance between resonators. Figure [4-17] is a A 
Monte Carlo test environment was set up in ADS. The open gap of each loop resonator is set to be $\mathrm{g} 1, \mathrm{~g} 2$, g3, g4, the gap distance between every two resonators are set to be s12, s14, s23, s34. The tapped line positions are $\mathrm{T} 1$ and $\mathrm{T} 4$. All parameters are all set to be Gaussian random variables with a 5\% standard deviation shown in Figure [4-18]

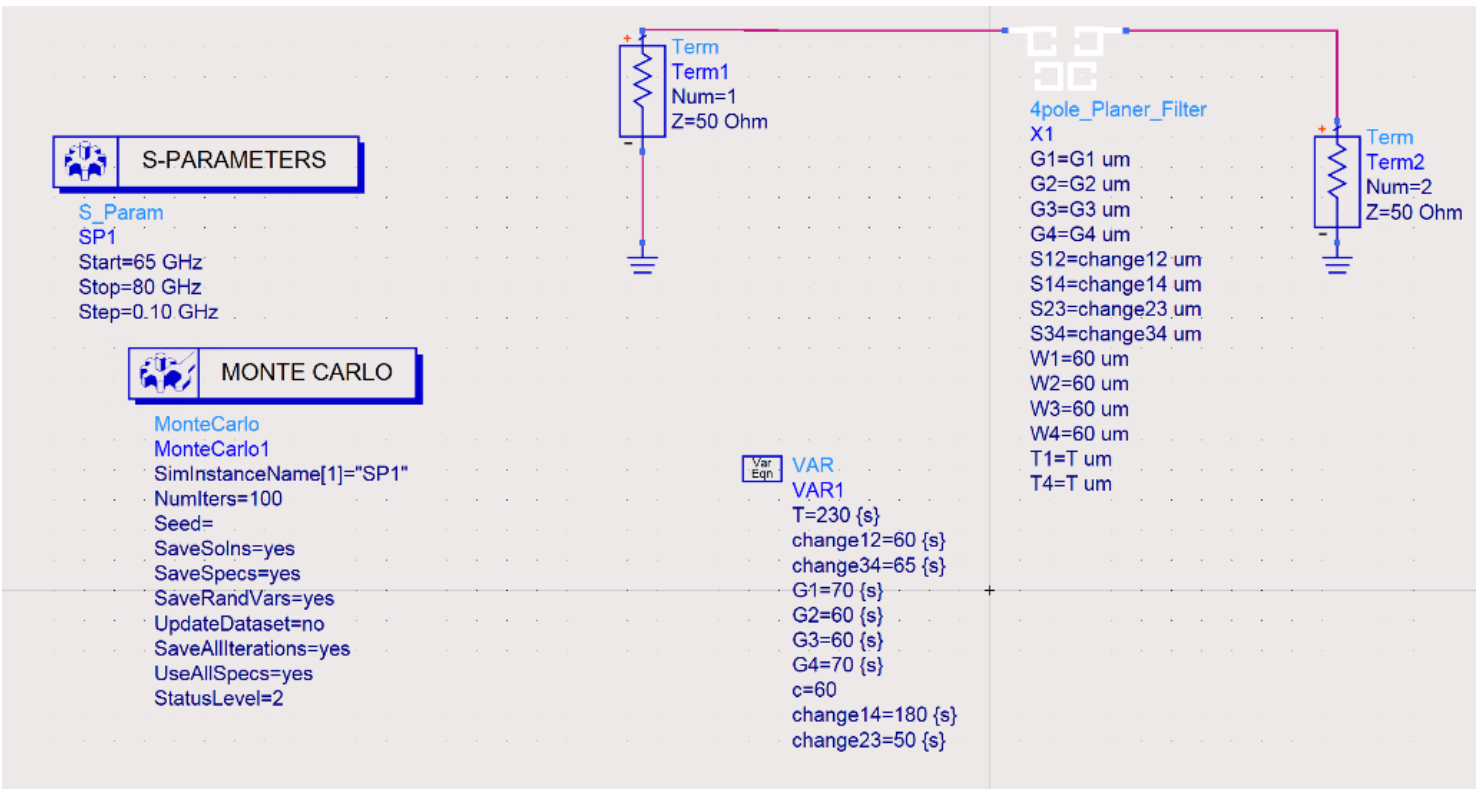

Figure 4-17 Monte Carlo analysis of the fourth-order filter

Figure [4-18] shows the results of 1000 trials of Monte Carlo analysis. It shows that this filter's frequency response is relatively insensitive to the variation of these parameters. The blue line is $-5 \mathrm{~dB}$ insertion loss. For most cases, it can still maintain the desired insertion loss. 


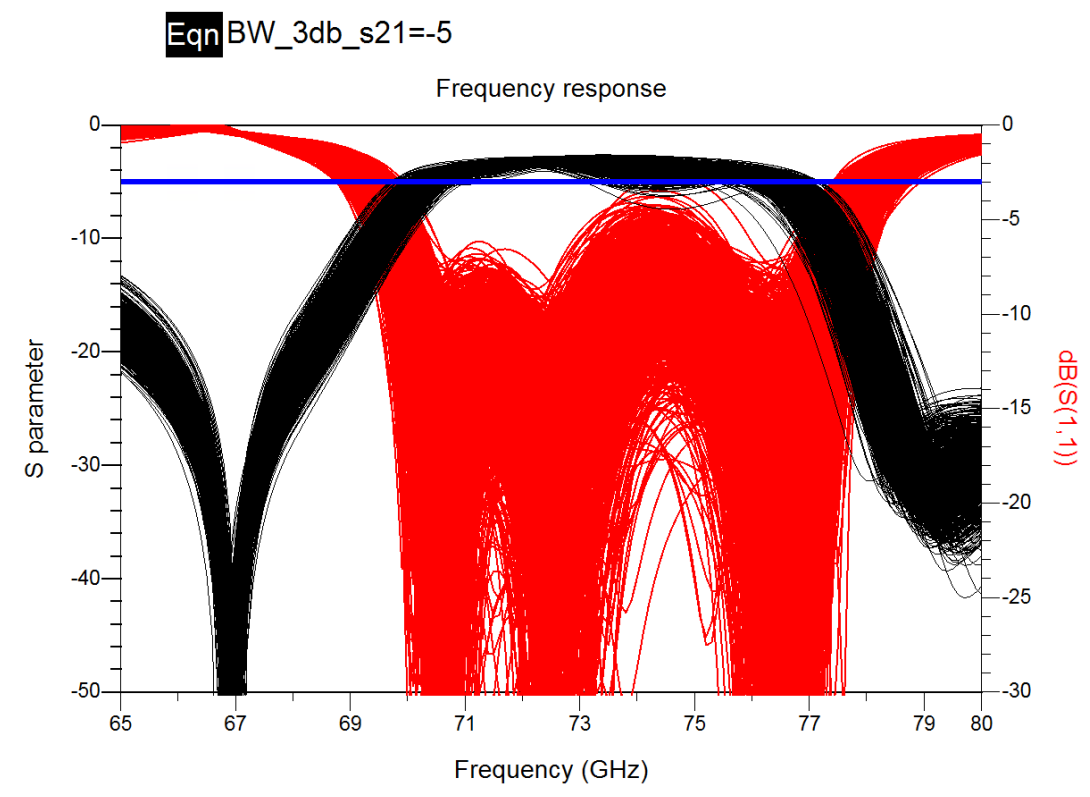

Figure 4-18 1000 trials of a fourth-order filter Monte Carlo analysis

Figure [4-19] is a Monte Carlo test environment for a six-pole filter set up in ADS. Since it has two additional resonators, two additional open gaps G5 and G6, and two additional distance S12, S56 are added into the analysis.

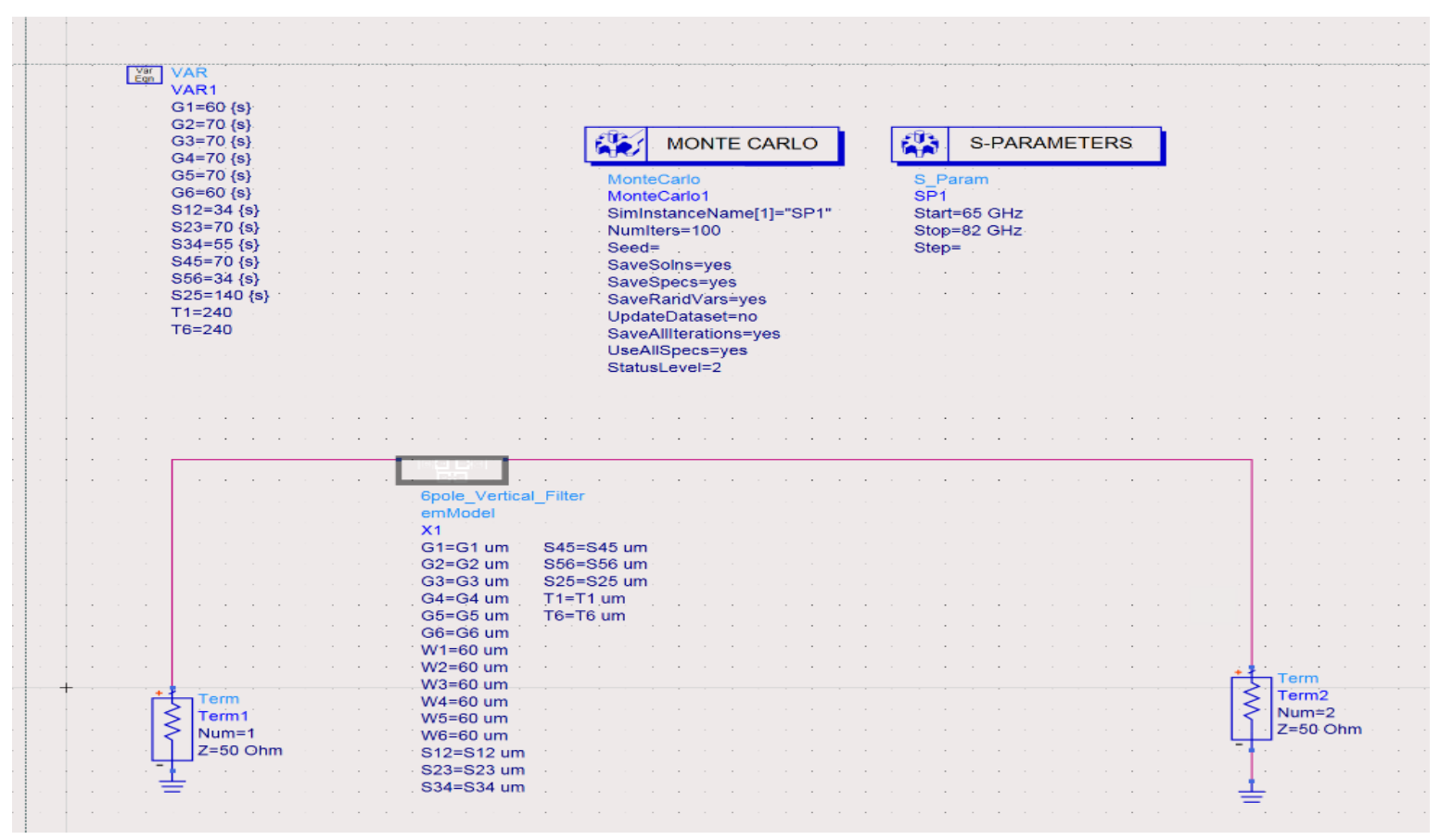

Figure 4-19 Monte Carlo Analysis for the sixth order filter 
Figure [4-20] is the results of 1000 trials of Monte Carlo analysis. The outcome shows that the sixth order filter's frequency response is also quite stable against parameter variations.

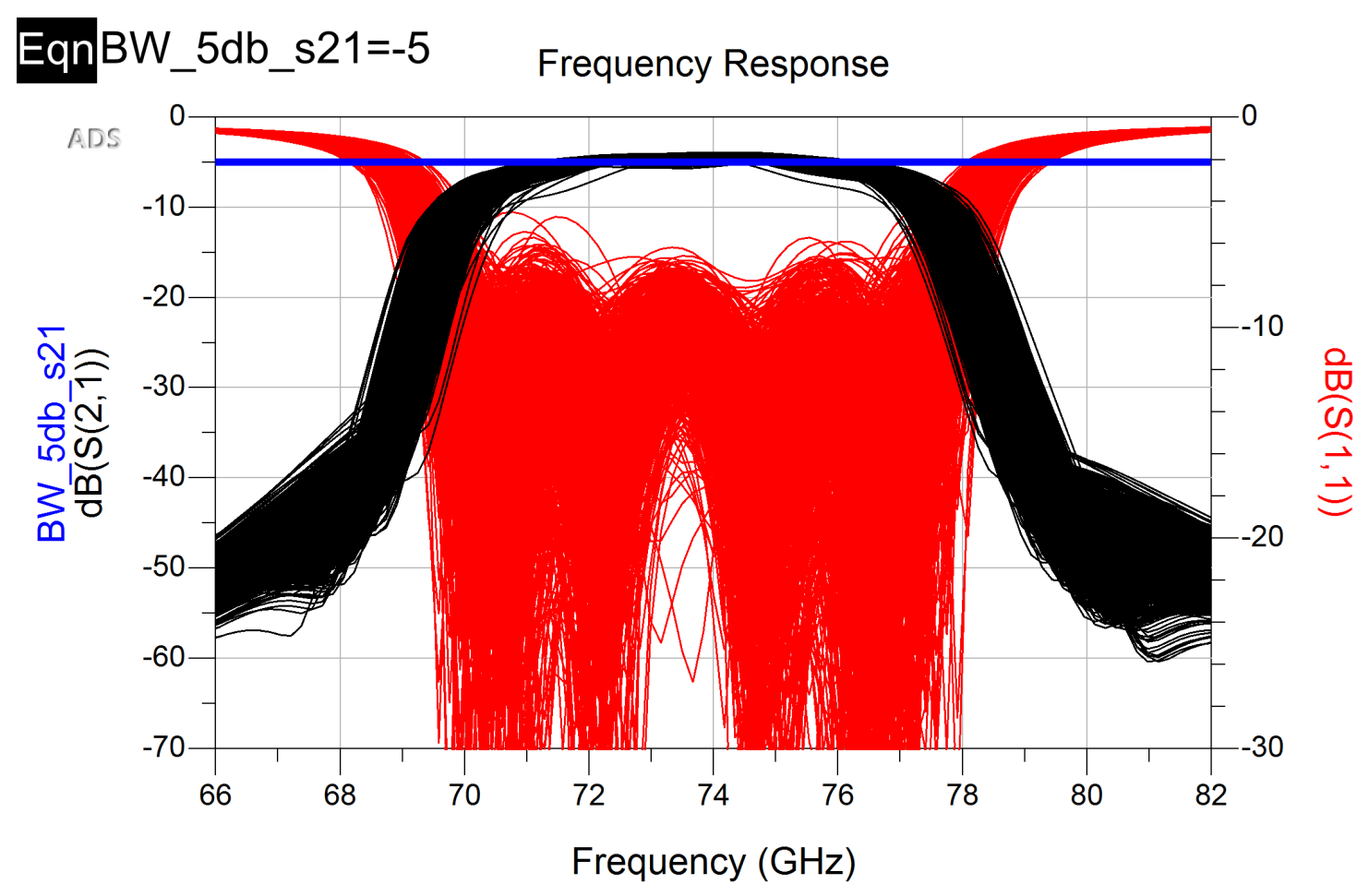

Figure 4-20 1000 trials of sixth-order filter Monte Carlo analysis

\subsection{Summary}

This chapter describes the details of narrow bandwidth millimeter-wave filter design in an

LTCC module. The simulation results indicate that normal planar filters will suffer from a high conductor loss, which makes them not an optimum option for filter implementation. However, the disadvantage of high conductor loss of the planar filters using conventional conducting thin films can be overcome by replacing them with high-temperature superconducting (HTS) thin films. These can have a very low conductor loss[22], [23]. 


\section{Chapter 5: Measurement}

\subsection{Fabrication and measurement}

Since the E-band filter could not be measured, as a substitution, another bandpass filter operating at $10 \mathrm{GHz}$ was fabricated for measurement and validation purpose. The filter is realized using the same configuration in Figure [4-1] and fabricated using a microstrip structure on a Roger4360G2 substrate which has a relative dielectric constant of 6.15 and a thickness of $0.508 \mathrm{~mm}$. The filter dimensions are determined based on the same full-wave EM simulation method in Chapter 4. Due to the change of dielectric constant and frequency, the size of each resonator and the coupling coefficient between every two resonators need to be readjusted. Design equations for the microstrip line are also given in Chapter 2. Figure [5-1] depicts the physical structure of this fourth-order filter. The size of the filter is about "7mm x 7mm".

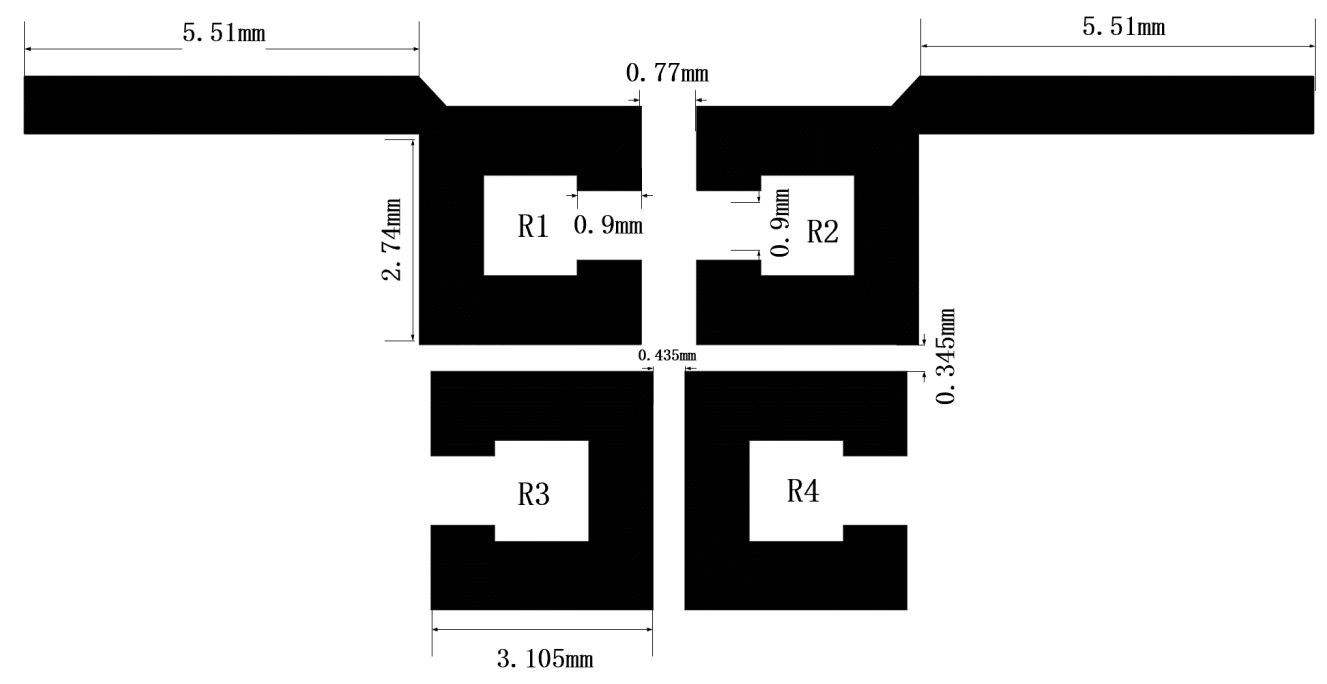

Figure 5-1 10GHz Planar Filter 
This filter is fabricated by an in-house circuit board plotter LPKF Protomat S100 [24]. Figure [5-3] is a picture of the machine. It consists basically of a base plate, an X/Y motion system, and a machining head. This machining head can be moved along both axes with the aid of the $\mathrm{X} / \mathrm{Y}$ motion system. The machining head can also be lowered. A channel can be milled with a milling cutter in a piece of base material fastened to the base plate by moving the lower machining head. However, the depth of the milling cutter must be manually adjusted. And this introduced some inaccuracy that was critical to the design.

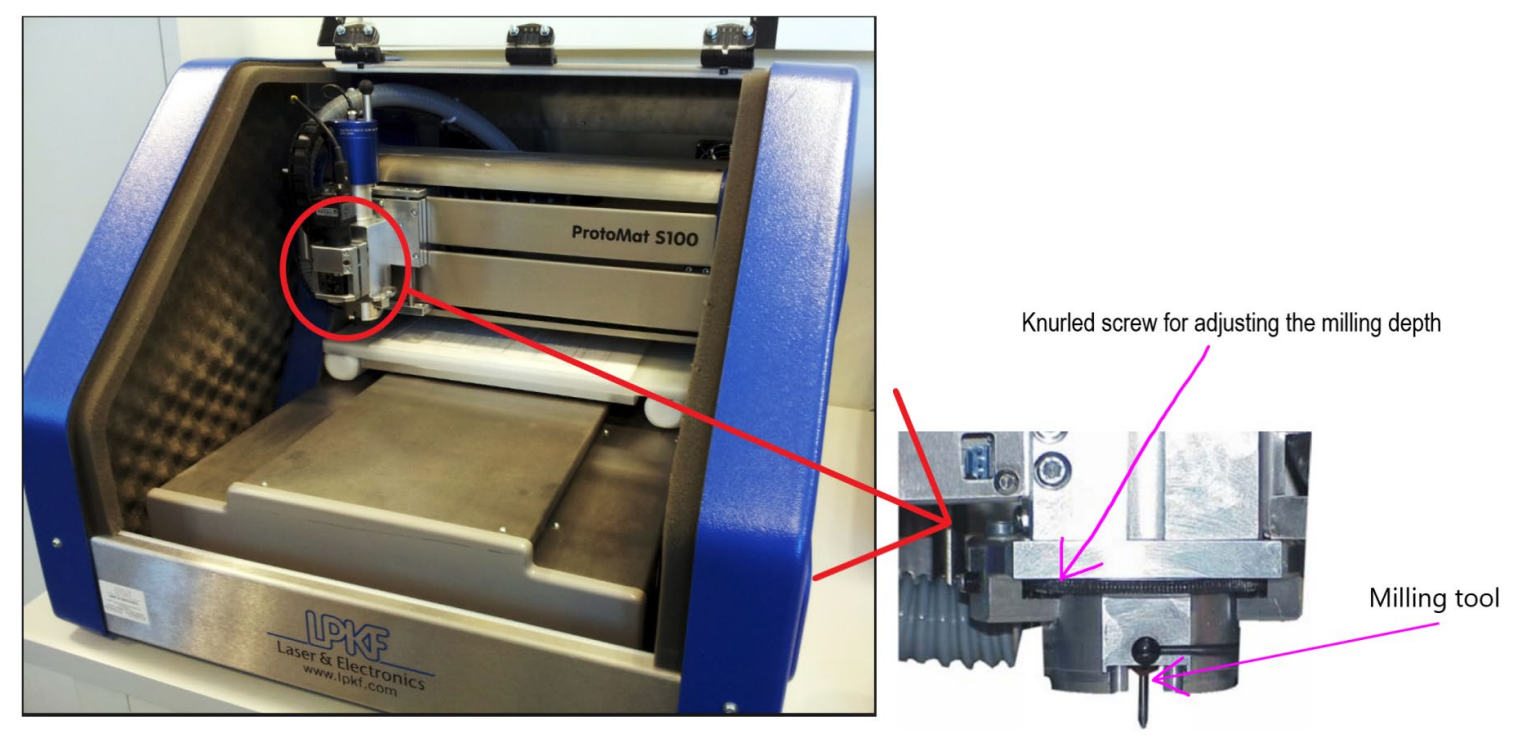

Figure 5-2 LPFK Protomat S100[24]

The machine is controlled and operated completely by the BoardMaster software, so the design file must be first implemented into the software for preparation. Before the machine starts to mill the top layer, the milling tool must be adjusted to a certain height manually. Figure [5-4] is a photograph of the fabricated filter. The starting material is metal-substratemetal. In order to make the filter, all the top layer metal must be removed except for the resonators and the feeds. However, the milling tool must be set deep enough to remove all 
the metal and so it will remove some substrate as well. This additional removed substrate material is not initially modeled.

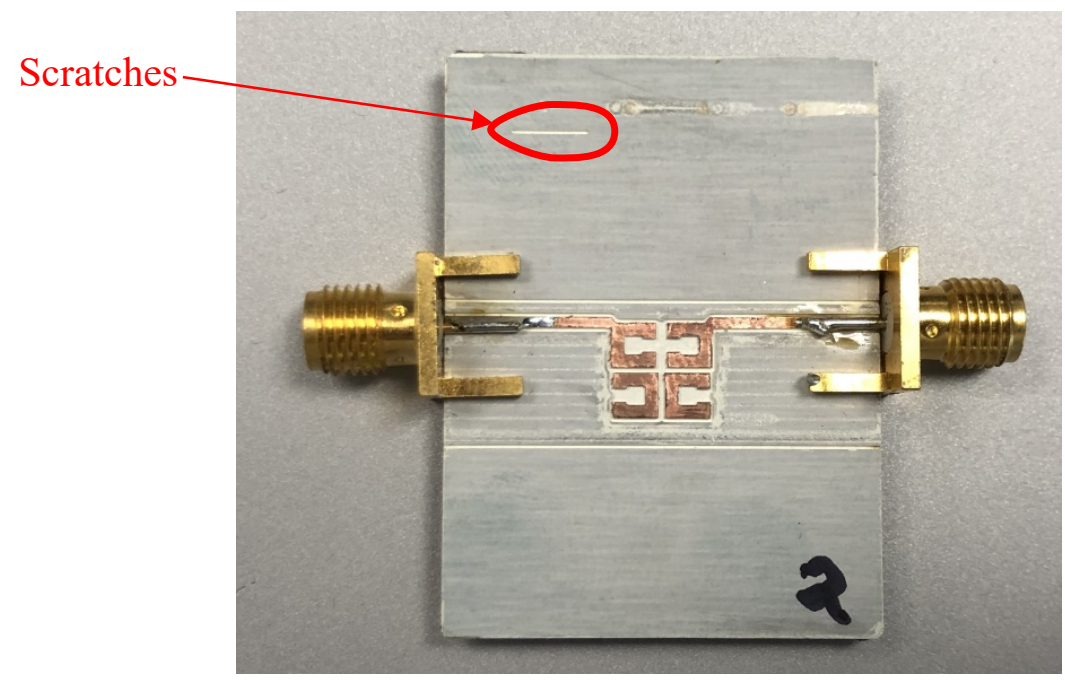

Figure 5-3 Fabricated 10GHz filter

The fabricated filter is measured on an N5225A VNA network analyzer with an electronic calibration module used to calibrate losses and phase delays caused by the effects of the connectors, cables, and transitions.
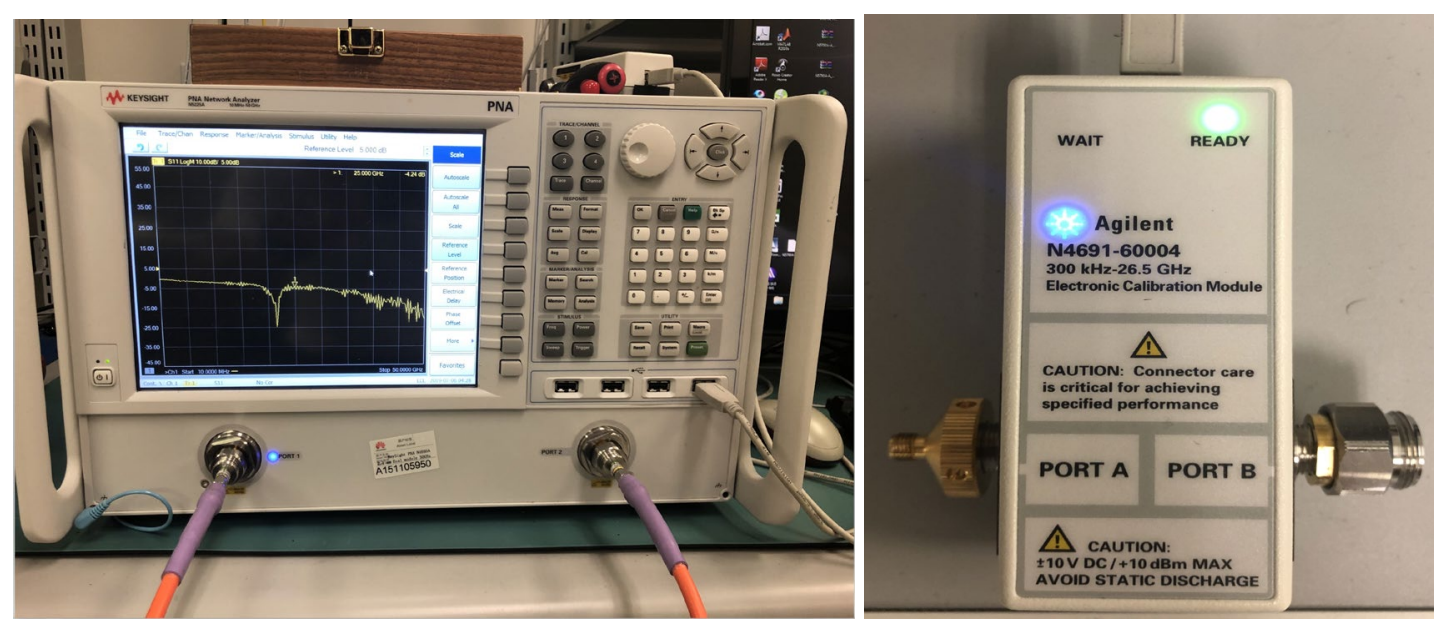

Figure 5-4 Test equipment and calibration module

The measurement result compared with the simulation result is shown in Figure [5-5]. It shows that the fabricated filter center frequency is shifted to $11.19 \mathrm{GHz}$. The mid-band 
insertion loss is about $-6 \mathrm{~dB}$ with a $2 \mathrm{~dB}$ ripple level. The mid-band return loss is worse than $-5 \mathrm{~dB}$, which indicates that the fabricated filter is totally mismatched. The two attenuation poles near the cut-off frequencies of the passband can still be identified, but they also shift to a higher frequency.

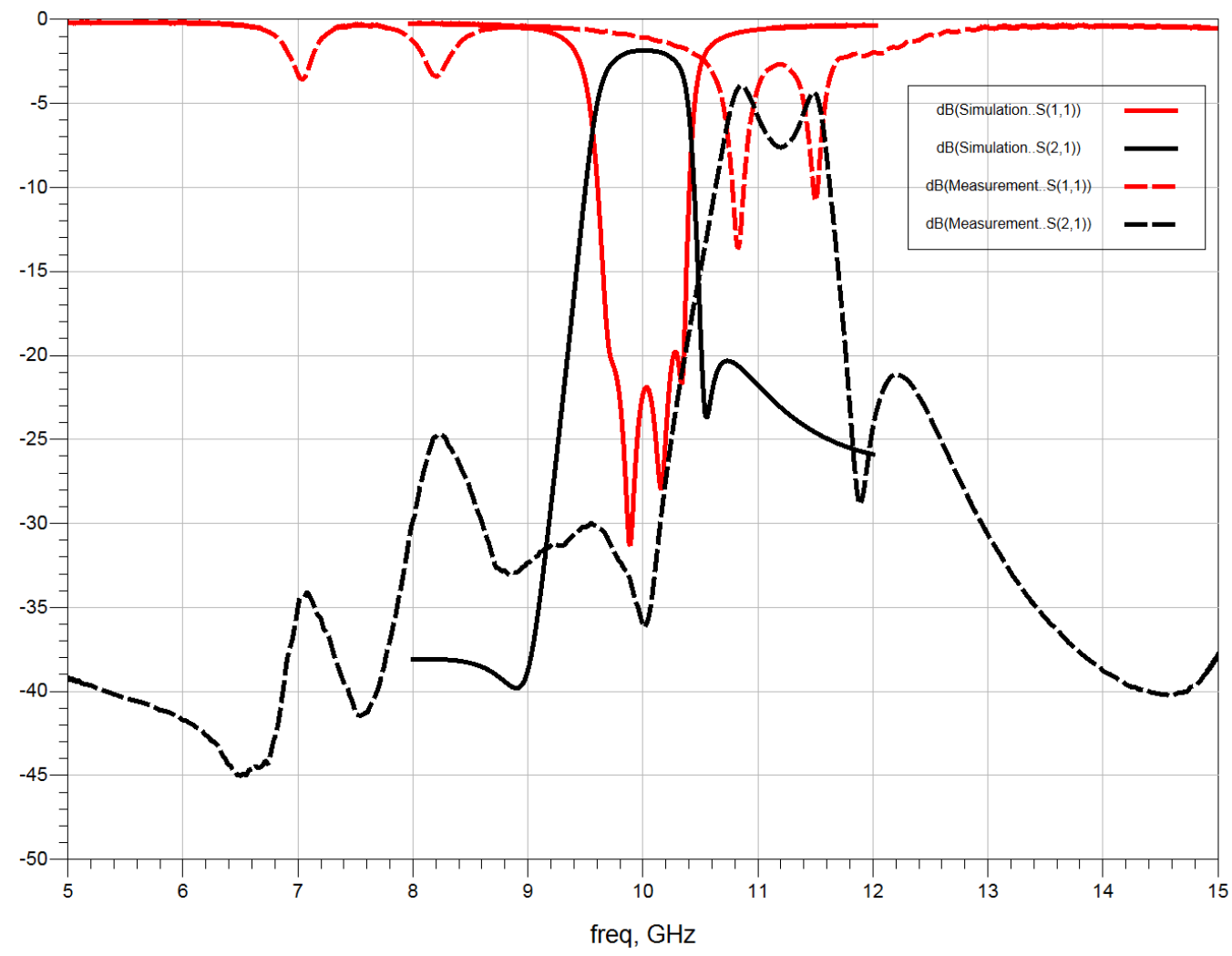

Figure 5-5 Simulation Versus Measurement

\subsection{Analysis of performance}

\begin{tabular}{|c|c|c|c|c|c|c|c|}
\hline & $\mathrm{F}_{\mathrm{c}}$ & $\mathrm{F}_{\mathrm{l}}$ & $\mathrm{F}_{\mathrm{h}}$ & $\mathrm{BW}$ & $\mathrm{F}_{\mathrm{z} 1}$ & $\mathrm{~F}_{\mathrm{z} 2}$ & $\mathrm{~S}_{21}$ \\
\hline Simulation & $10 \mathrm{GHz}$ & $9.65 \mathrm{GHz}$ & $10.38 \mathrm{GHz}$ & $7.3 \%$ & $8.74 \mathrm{GHz}$ & $10.65 \mathrm{GHz}$ & $<-3 \mathrm{~dB}$ \\
\hline Measurement & $11.19 \mathrm{GHz}$ & $10.84 \mathrm{GHz}$ & $11.49 \mathrm{GHz}$ & $5.8 \%$ & $10 \mathrm{GHz}$ & $11.9 \mathrm{GHz}$ & $<-7 \mathrm{~dB}$ \\
\hline
\end{tabular}

Table 5-1 Simulation Versus Measurement 
Table [5-1] summarizes the measurement result and the simulation result. Actual test results and simulation results show substantial discrepancies. The possible explanation is due to the milling depth of the fabrication machine.

As mentioned above in Figure [5-2], the milling depth of the machine must be adjusted manually, and this will change the resonator's frequency. In Figure [5-6], an HFSS model is created trying to model the effect of this milling depth. A cavity around the filter structure is created to simulate the relation between different milling depth and the filter's frequency response. The simulation results are shown in Figure [5-7] and Figure [5-8].

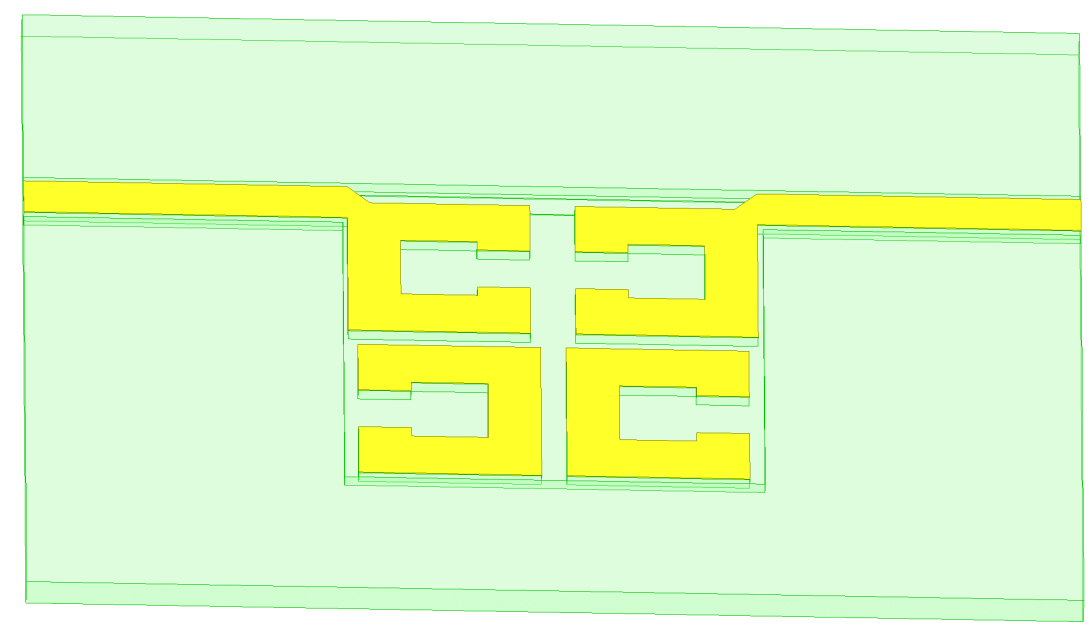

Figure 5-6 Modelling edge effect in HFSS 


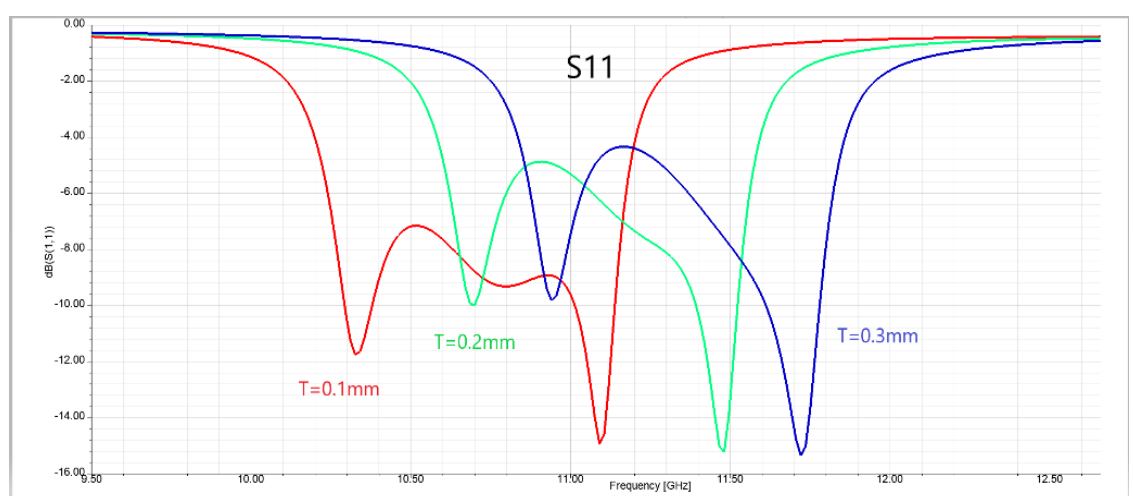

Figure 5-7 S11 for milling depth from $0.1 \mathrm{~mm}$ to $0.3 \mathrm{~mm}$

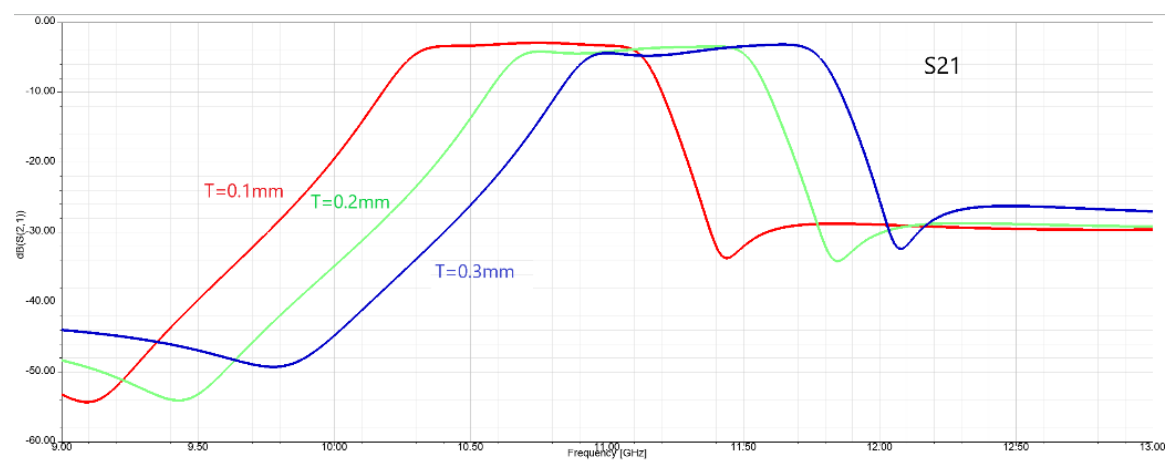

Figure 5-8 S21 for milling depth from $0.1 \mathrm{~mm}$ to $0.3 \mathrm{~mm}$

From the figure shown above, as the milling depth becomes deeper, the return loss gets worse, and the center frequency moves toward a higher frequency.

For a more accurate analysis, the actual milling depth of the fabricated filter is measured by a 3D optical profilometer. Figure [5-9] is the measurement result. The milling depth is the distance between the substrate and the cutting edge, which is $0.243 \mathrm{~mm}$ in the figure. The tapped line width is also measured and is shown in Figure [5-10]. The actual width is $0.743 \mathrm{~mm}$, which is close to $0.75 \mathrm{~mm}$ in the original simulation. 


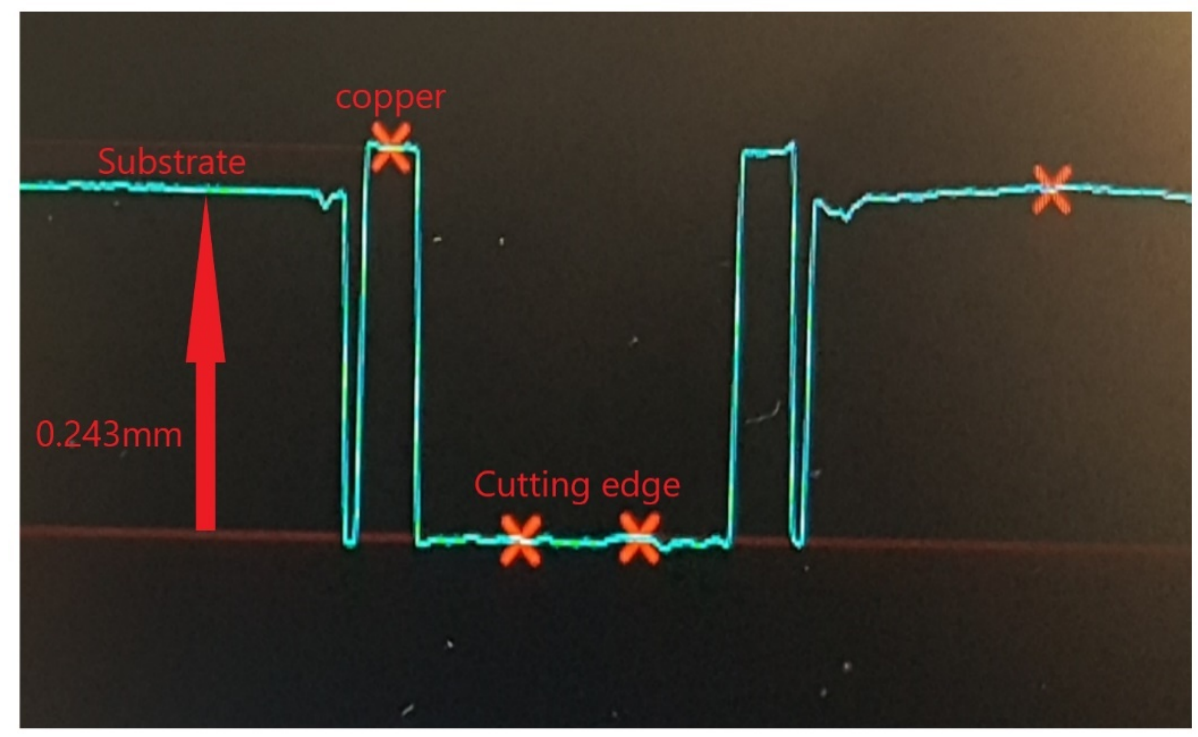

Figure 5-9 Measuring the milling depth of the fabricated filter

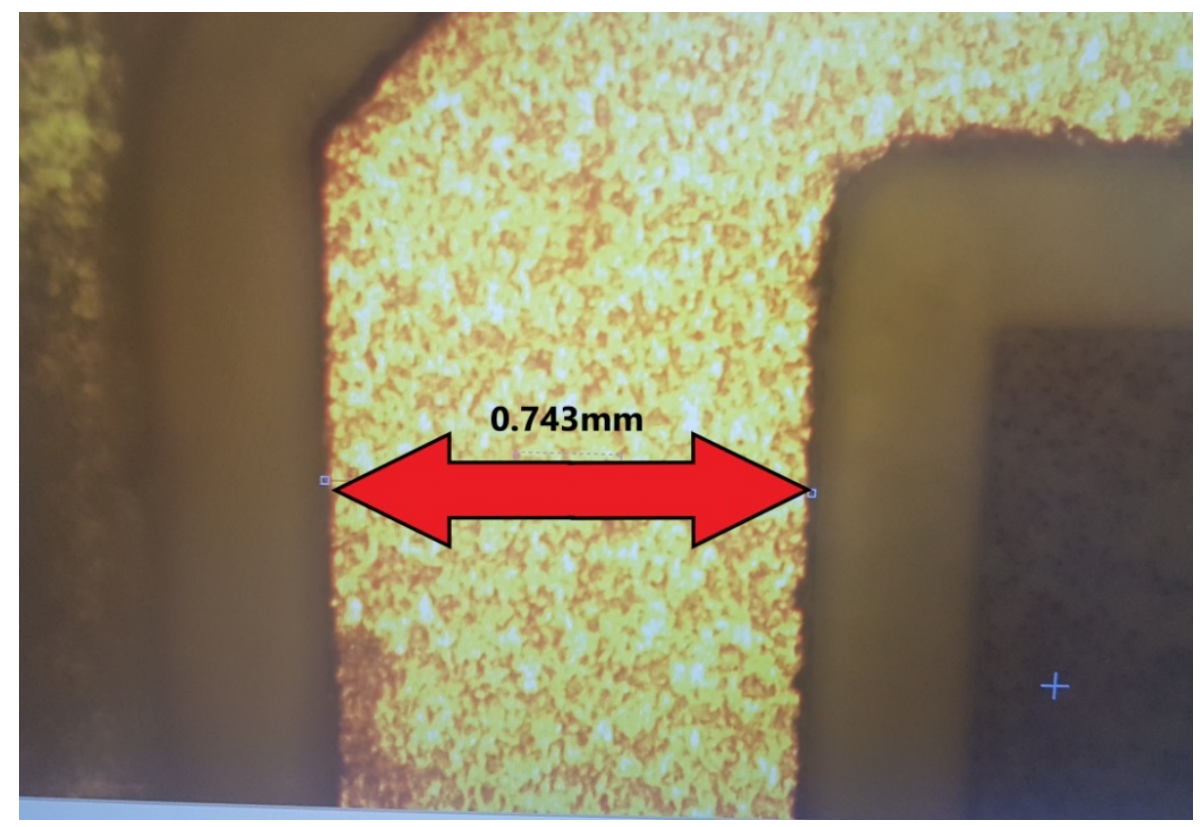

Figure 5-10 Measurement of conductor width

Adding a $0.243 \mathrm{~mm}$ milling depth into the simulation, the comparison of the simulation result and the real measurement result is in Figure [5-11]. The results show consistency. 


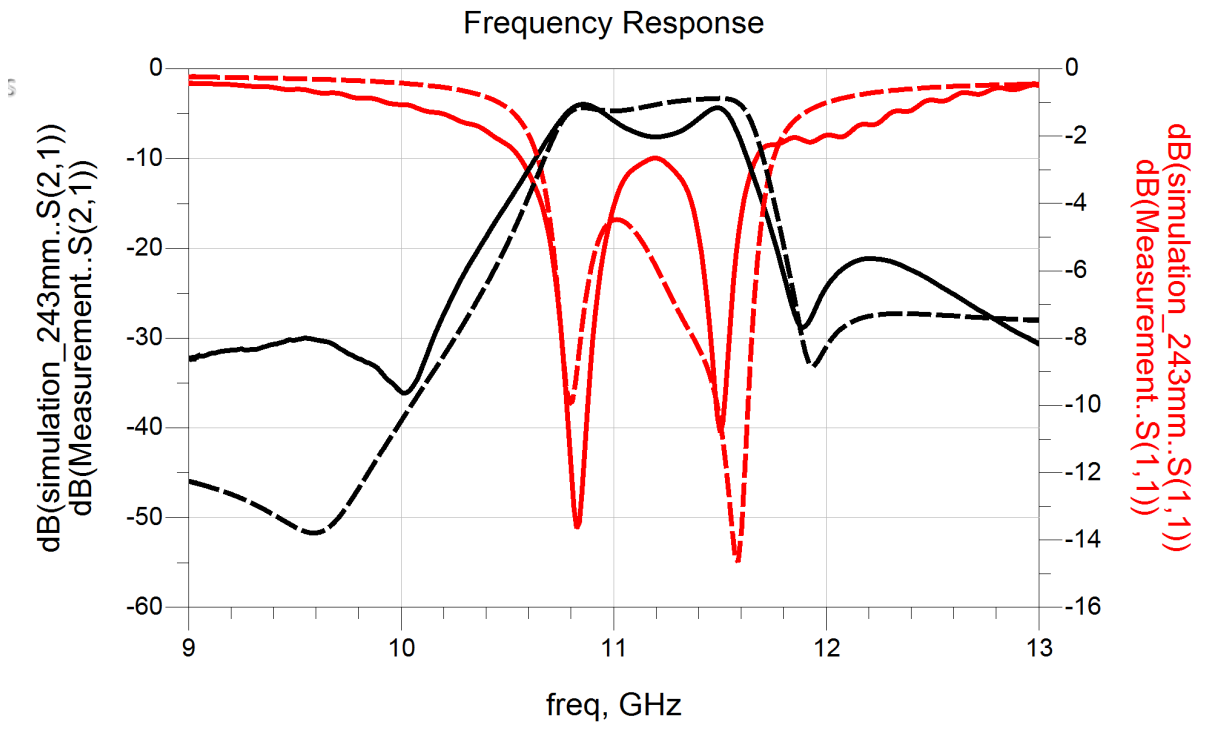

Figure 5-11 Filter with $0.243 \mathrm{~mm}$ milling depth simulation result compare with the measurement result

This proves our theory that the milling depth is the main reason for the frequency shift and impedance mismatch observed during measurement. If the filter would have been implemented in a standard PCB fabrication process, we have reason to believe the measurement result will show consistency with the original simulation result. 


\section{Chapter 6: Conclusion}

\subsection{Summary}

In this thesis, two planar bandpass filters operating at $70 \mathrm{GHz}$ are designed. They both exhibit a single pair of attenuation poles at finite frequencies. The filter's fractional bandwidth is $7.8 \%$. This type of filter can greatly improve frequency selectivity while at the same time, maintaining a lower filter order. The out-of-band attenuation at 0.05 offset frequency is more than $25 \mathrm{~dB}$. The implementation of planar open-loop resonators not only allows the cross-coupling to be realized simpler but also makes the filters compact. Also, this design technique is, of course, not limited to the application of microstrip or stripline filters and, hence, it can be applied to design a filter using other structures. The full-wave EM simulations have been carried out to determine the filter dimensions.

A second filter, operating at $10 \mathrm{GHz}$, was fabricated with Roger4360 material. The measurement result has some deviation with the simulation result since during the fabrication process, part of the original substrate is trimmed, causing shifts at each resonator's center frequency. The final simulation result includes this effect and shows a strong agreement with the measured results.

\subsection{Thesis Contribution}

The primary contributions of this thesis to the Millimeter-wave filter design can be summarized as follows: 
- Presented a novel vertical coupling structure of the six-order filter indicates that multilayer technology can be applied to overcome the fabrication limit on the planar filter. By putting two resonators on two different layers in an LTCC module, the required distance to get the coupling coefficient can be achieved with no horizontal limitation.

- The design and fabrication of a $10 \mathrm{GHz}$ filter which uses Rogers material have been presented. The measurement result is not matched with the simulation result since the thickness of the substrate has been trimmed during the fabrication process. Simulation result with the trimmed substrate is in strong agreement with the measurement result.

- Experimental validation indicates that the planar filter in an LTCC module can be achieved at E-band frequency. The insertion loss is normally around 4-5dB, which is mainly due to conductor loss. The thickness of the substrate will influence filter performance and need to be treated carefully.

\subsection{Future work}

There are several areas left for future improvement. First, the conductor loss of the planar filter can be reduced by the substitution of substrate integrated waveguide (SIW).[25] The conductor loss would increase as the frequency goes higher. At E-band frequency, the dissipation loss through a conductor is much higher compared with dielectric loss. By substituting conductor to SIW, there will be no surface current, and this would largely decrease the insertion loss. 
Another improvement is to design a cascade of the filter in Figure [4-1]. Each fourth-order filter can be considered as a basic unit. By cascading each of them one by one, several outof-band transmission zeros could be achieved to further improve the filter selectivity.

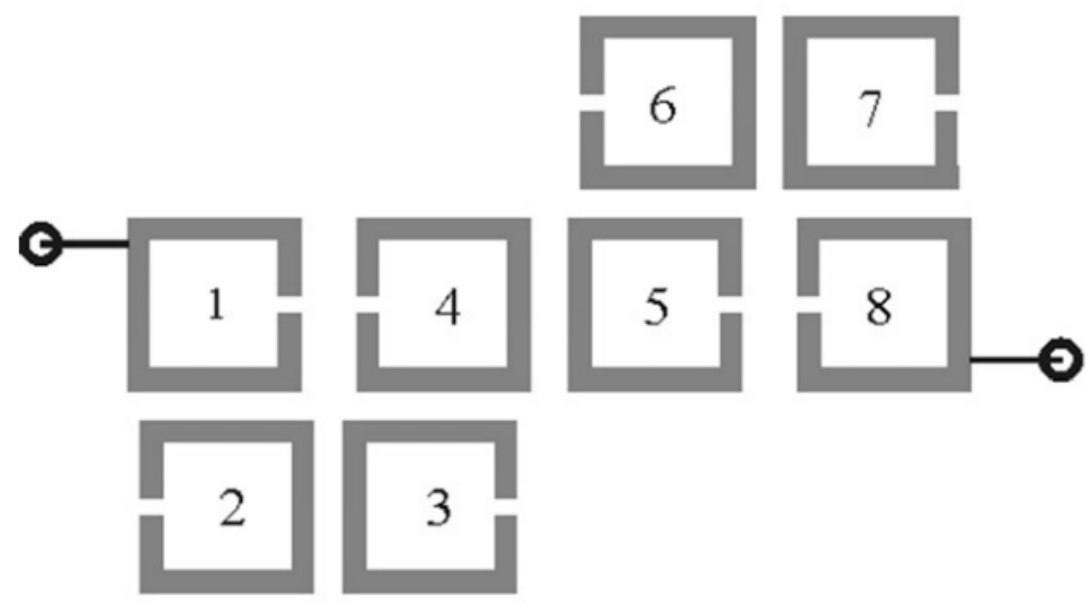

Figure 6-1 cascade of two fourth-order filters

Figure [6-1] is a cascade structure of two four-order filters. Two pair of transmission zeros could be achieved. Figure [6-2] is the frequency response of this filter. Two pair of transmission zeros next to the passband could further improve the filter selectivity. The disadvantage of this structure is that the conductor loss would increase due to the higher filter order.

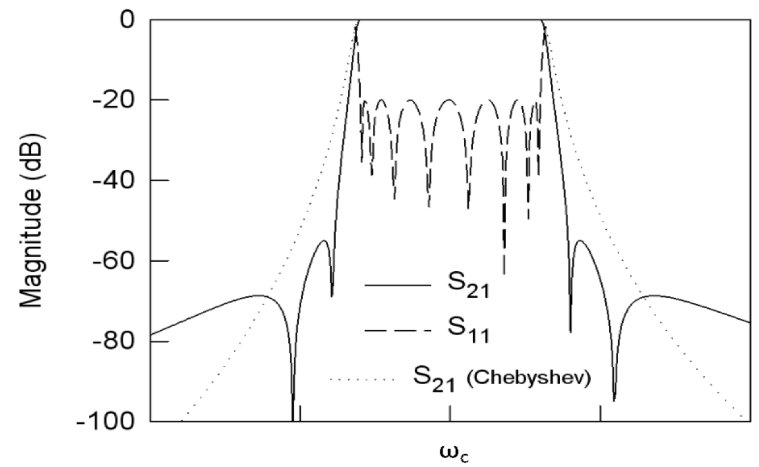

Figure 6-2 Frequency response of the filter with two pair of transmission zero 


\section{Reference}

[1] "Cisco visual networking index (VNI) global mobile data traffic," white Pap. Cisco.com, 2014.

[2] J. G. Andrews et al., "What will 5G be?" IEEE J. Sel. Areas Commun., vol. 32, no. 6, pp. 1065-1082, 2014.

[3] W. Roh et al., "Millimeter-Wave Beamforming As An Enabling Technology For 5g Cellular Communications: Theoretical feasibility and prototype results," IEEE Commun. Mag., vol. 52, no. 2, pp. 106-113, 2014.

[4] D. M. Pozar, Microwave Engineering, 4th ed. 2012.

[5] Z. Qingling and J. Li, "Rain Attenuation in Millimeter Wave Ranges," IEEE, 2007.

[6] S. Rangan, T. S. Rappaport, and E. Erkip, "Millimeter-Wave Cellular Wireless Networks: Potentials And Challenges," Proc. IEEE, vol. 102, no. 3, pp. 366-385, 2014.

[7] F. Gutierrez and S. M. Ieee, "State of the Art in 60-GHz Integrated Circuits and Systems for Wireless Communications," Proc. IEEE (Volume99, Issue 8), vol. 99, no. $8,2015$.

[8] V. Miraftab, W. Zhai, and M. Repeta, “A Wideband Low-Cost E-Band Siw Antenna Array For High Capacity Mmwave Radio,” 2015 IEEE MTT-S Int. Microw. Symp. IMS, 2015, pp. 1-3, 2015.

[9] W. Zhai, M. Repeta, D. Wessel, and W. Tong, "Mm-Wave Large-Scale Phased Array Based On Randomly Tiled Rectangular Sub-Arrays For 5G Communications," IEEE MTT-S Int. Microw. Symp. Dig., pp. 1895-1898, 2017. 
[10] K. Nishikawa, T. Seki, I. Toyoda, and S. Kubota, “Compact 60-GHz LTCC Stripline Parallel-Coupled Bandpass Filter With Parasitic Elements For Millimeter-Wave System-On-Package." in IEEE MTT-S International Microwave Symposium Digest, 2007.

[11] Geroge. L. Matthaei, 1. Young, and e. M. T. Jones, Microwave Filters, ImpedanceMatching Networks, and Coupling Structures. 2000.

[12] J. S. Hong and M. J. Lancaster, "Design of Highly Selective Microstrip Bandpass Filters with a Single Pair of Attenuation Poles at Finite Frequencies," IEEE Trans. Microw. Theory Tech., vol. 48, no. 7 PART 1, pp. 1098-1107, 2000.

[13] R. Levy, "Filters with Single Transmission Zeros at Real or Imaginary Frequencies," IEEE Trans. Microw. Theory Tech., vol. 24, no. 4, pp. 172-181, 1976.

[14] K. T. Jokela, "Narrow-band Stripline or Microstrip Filters with Transmission Zeros at Real and Imaginary Frequencies," IEEE Trans. Microw. Theory Tech., vol. M. No. 6, pp. 542-547, 1980.

[15] Jia-sheng Hong, Microstrip Filters for RF/Microwave Applications.

[16] R. Levy and S. B. Cohn, "A History of Microwave Filter Research, Design, and Development," IEEE Trans. Microw. Theory Tech., vol. 32, no. 9, pp. 1055-1067, 1984.

[17] M. J. Lancaster and J. S. Hong, "Realisation of quasielliptic function filter using dual-mode microstrip square loop resonators," Electron. Lett., vol. 31, no. 24, pp. 2085-2086, 2002. 
[18] S. J. Yao, R. R. Bonetti, and A. E. Williams, "Generalized Dual-Plane Multicoupled Line Filters," IEEE Trans. Microw. Theory Tech., vol. 41, no. 12, pp. 2182-2189, 1993.

[19] Rony. E. Amaya, A. Momciu, and I. Haroun, "High-performance, compact quasielliptic bandpass filters for V-band high data rate radios," IEEE Trans. Components, Package. Manuf. Technol., vol. 3, no. 3, pp. 411-416, 2013.

[20] J.-S. Hong and M. J. Lancaster, "Coupling of Microstrip Open-Loop Resonators for Cross-Coupled Planar Microwave Filters," Ieee Trans. Microw. Theory Tech., vol. 44, no. 12, pp. 2099-2109, 1996.

[21] Advanced Design System: ADS2009U1-Momentum, Agilent Technologies, Santa Clara, Ca, 2009

[22] S. J. Hedges, W. H. Road, G. Baddow, R. G. Humphreys, E. Division, and G. Malvern, "An Extracted Pole Microstrip Elliptic Function Filter Using HighTemperature Superconductors" in 24th European Microwave Conference, 1994, vol. 189, no. 1986, pp. 517-521.

[23] G. L. Matthaei and G. L. Hey-Shipton, "Novel Staggered Resonator Array Superconducting 2.3-GHz Bandpass Filter," IEEE Trans. Microw. Theory Tech., vol. 41, no. 12, pp. 2345-2352, 1993.

[24] LPKF: Manual ProtoMat S100, LPKF Laser \& Electronics AG, 2006

[25] T. Martin, A. Ghiotto, F. Lotz, and T. P. Vuong, "Air-Filled SIW Filters for K-to EBand Substrate Integrated Systems," 2018 IEEE MTT-S Int. Conf. Numer. Electromagn. Multiphysics Model. Optim. NEMO, 2018, no. 4, pp. 4-6, 2018. 